Bull. Soc. math. France

132 (2), 2004, p. 157-199

\title{
ÉLÉMENTS RÉGULIERS ET REPRÉSENTATIONS DE GELFAND-GRAEV DES GROUPES RÉDUCTIFS NON CONNEXES
}

\author{
PAR KARINE SORLIN
}

RÉSUMÉ. - Soient $G$ un groupe algébrique réductif connexe défini sur $\mathbb{F}_{q}$ et $F$ l'endomorphisme de Frobenius correspondant. Soit $\sigma$ un automorphisme rationnel quasicentral de $G$. Nous construisons ci-dessous l'équivalent des représentations de GelfandGraev du groupe $\widetilde{G}^{F}=G^{F} \cdot\langle\sigma\rangle$, lorsque $\sigma$ est unipotent et lorsqu'il est semi-simple. Nous montrons de plus que ces représentations vérifient des propriétés semblables à celles vérifiées par les représentations de Gelfand-Graev dans le cas connexe en particulier par rapport aux éléments réguliers.

Texte reçu le 17 décembre 2002, accepté le 7 mars 2003

KARIne Sorlin, LAMFA, Université de Picardie Jules Verne, 33 rue Saint-Leu, 80000 Amiens (France) • E-mail : karine_sorlin@yahoo.fr • Url : http://www.mathinfo.upicardie.fr/sorlin/

Classification mathématique par sujets (2000). — 20C33, 20 G05.

Mots clefs. - Groupes réductifs finis, groupes algébriques non connexes. 
ABstRact (Regular Elements and Gelfand-Graev Representations for Disconnected Reductive Groups)

Let $G$ be a connected reductive group defined over $\mathbb{F}_{q}$ and let $F$ be the corresponding Frobenius endomorphism. Let $\sigma$ be a quasi-central automorphism of $G$, which means that $\sigma$ is quasi-semi-simple (i.e. $\sigma$ stabilises $(T \subset B)$ where $T$ is a maximal torus included in a Borel subgroup $B$ of $G$ ) and $\operatorname{dim}\left(G^{\sigma}\right)>\operatorname{dim}\left(G^{\sigma^{\prime}}\right)$ for any quasi-semisimple automorphism $\sigma^{\prime}=\sigma \circ \operatorname{ad}(g)$, where ad $(g)$ is the conjugation by $g$ for all $g \in G$. We suppose also that $\sigma$ is rational.

We define in this article Gelfand-Graev representations for the group $\widetilde{G}^{F}=G^{F} \cdot\langle\sigma\rangle$ when $\sigma$ is unipotent and when it is semi-simple, which extend the $\sigma$-stable GelfandGraev representations for connected reductive groups.

Let $T$ be a $\sigma$-stable rational maximal torus of $G$ included in a $\sigma$-stable rational Borel subgroup of $G$. Let $U$ be the unipotent radical of $B$.

In the connected reductive case, Gelfand-Graev representations of $G^{F}$ are obtained by inducing an irreducible linear character of $U^{F}$ which is called a regular character. We define a regular character of $U^{F} \cdot\langle\sigma\rangle$ as the extension of a $\sigma$-stable regular character of $U^{F}$. When $\sigma$ is unipotent, $\sigma$-stable Gelfand-Graev representations of $G^{F}$ are obtained by inducing $\sigma$-stable regular characters of $U^{F}$. In this case, we define Gelfand-Graev representations of $G^{F} \cdot\langle\sigma\rangle$ as the representations obtained by inducing regular characters of $U^{F} \cdot\langle\sigma\rangle$. When $\sigma$ is semi-simple, the definition of Gelfand-Graev representations is more complicated.

Gelfand-Graev representations of $G^{F} \cdot\langle\sigma\rangle$ have similar properties to Gelfand-Graev representations of $G^{F}$. They are multiplicity free and their Harish-Chandra restrictions to a rational $\sigma$-stable Levi subgroup included in a rational $\sigma$-stable parabolic subgroup still are Gelfand-Graev representations. We say that an element of $G \cdot \sigma$ is regular if the dimension of its centralizer in $G$ is minimal among all elements of $G \cdot \sigma$. The dual of any Gelfand-Graev representation of $G^{F} \cdot \sigma$ is zero outside regular unipotent elements of $G^{F} \cdot \sigma$ when $\sigma$ is unipotent (resp. outside regular pseudo-unipotent elements of $G^{F} \cdot \sigma$, i.e. conjugates under $G$ of regular elements of $U \cdot \sigma$, when $\sigma$ is semi-simple). Moreover, Gelfand-Graev representations can be used to calculate the average value of irreducible characters of $G^{F} \cdot\langle\sigma\rangle$ on the set of $G^{F}$-classes of regular unipotent (resp. pseudo-unipotent) elements of $G^{F} \cdot \sigma$ if $\sigma$ is unipotent (resp. semi-simple). When $\sigma$ is semi-simple, the characteristic can be chosen good for $\left(G^{\sigma}\right)^{0}$ and we can get the exact values of irreducible characters of $G^{F} \cdot\langle\sigma\rangle$ on $G^{F}$-classes of regular pseudo-unipotent elements of $G^{F} \cdot \sigma$.

\section{Introduction}

Les groupes réductifs non connexes de la forme $G \cdot\langle\sigma\rangle$, où $G$ est un groupe réductif connexe et $\sigma$ un automorphisme de $G$, ont été étudiés par R. Steinberg [8], F. Digne \& J. Michel [4] et G. Malle [6].

On suppose que $G$ est un groupe réductif connexe défini sur $\mathbb{F}_{q}$, que $F$ est l'endomorphisme de Frobenius correspondant et que $\sigma$ est un automorphisme quasi-central de $G$, ce qui signifie que $\sigma$ est quasi-semi-simple (i.e. $\sigma$ stabilise un couple $(T \subset B)$ où $T$ est un tore maximal inclus dans un sous-groupe de

TOME $132-2004-\mathrm{N}^{\mathrm{O}} 2$ 
Borel $B$ de $G)$ et $\operatorname{dim}\left(G^{\sigma}\right)>\operatorname{dim}\left(G^{\sigma^{\prime}}\right)$ pour tout automorphisme quasi-semisimple $\sigma^{\prime}=\sigma \circ \operatorname{ad}(g)$, où $\operatorname{ad}(g)$ est la conjugaison par $g$ pour tout $g \in G$. On suppose de plus $\sigma$ rationnel.

La notion d'élément quasi-central a été introduite par F. Digne \& J. Michel dans [4]. Ils ont montré [4, prop. 1.34] que pour tout automorphisme rationnel $\sigma^{\prime}$ de $G$, il existe $g$ dans $G$ tel que $\sigma^{\prime} \circ \operatorname{ad}(g)$ soit rationnel quasi-central. Quand $\sigma^{\prime}$ est un automorphisme quasi-semi-simple de $G$, alors $G^{\sigma^{\prime}}$ est un groupe réductif $\left[4\right.$, th. 1.8] et quand $\sigma^{\prime}$ est quasi-central, il y a de nombreuses similarités entre $G \cdot \sigma^{\prime}$ et $\left(G^{\sigma^{\prime}}\right)^{0}$, en particulier pour les systèmes de racines et les groupes de Weyl [4, th. 1.15], les caractères de Deligne-Lusztig [4, section 4] et les représentations de Gelfand-Graev (section 5).

Le but de cet article est de définir des représentations de Gelfand-Graev pour $\widetilde{G}^{F}=G^{F} \cdot\langle\sigma\rangle$ qui étendent les représentations de Gelfand-Graev $\sigma$-stables pour les groupes réductifs connexes.

Soit $T$ un tore maximal rationnel $\sigma$-stable de $G$ inclus dans un sous-groupe de Borel rationnel $\sigma$-stable de $G$. Soit $U$ le radical unipotent de $B$. Dans le cas connexe, les représentations de Gelfand-Graev de $G^{F}$ sont obtenues en induisant certains caractères irréductibles de $U^{F}$ qu'on appelle caractères réguliers. Dans le cas non connexe, on définit un caractère régulier de $U^{F} \cdot\langle\sigma\rangle$ comme l'extension d'un caractère régulier $\sigma$-stable de $U^{F}$. Nous nous placerons dans un des deux cas extrêmes $\sigma$ unipotent ou $\sigma$ quasi-semi-simple. Le cas général peut s'y ramener en utilisant la décomposition de Jordan de $\sigma$.

Lorsque $\sigma$ est unipotent, les représentations de Gelfand-Graev $\sigma$-stables de $G^{F}$ sont obtenues en induisant un caractère régulier $\sigma$-stable de $U^{F}$ (proposition 5.1). Dans ce cas, on définit les représentations de Gelfand-Graev de $G^{F} \cdot\langle\sigma\rangle$ comme les représentations obtenues en induisant les caractères réguliers de $U^{F} \cdot\langle\sigma\rangle$ (définition 5.2). Quand $\sigma$ est semi-simple, la proposition 5.1 n'est plus vraie. La définition des représentations de Gelfand-Graev est alors plus compliquée (définition 5.3).

Les représentations de Gelfand-Graev de $G^{F} \cdot\langle\sigma\rangle$ ont les mêmes propriétés que les représentations de Gelfand-Graev de $G^{F}$. Leurs composantes irréductibles sont de multiplicité 1 (proposition 6.1). Leur restriction de HarishChandra à un sous-groupe de Levi $\sigma$-stable rationnel inclus dans un sous-groupe parabolique $\sigma$-stable rationnel est encore une représentation de Gelfand-Graev (proposition 6.4). On dit qu'un élément de $G \cdot \sigma$ est régulier si la dimension de son centralisateur dans $G$ est minimale parmi les éléments de $G \cdot \sigma$. Le dual de toute représentation de Gelfand-Graev de $G^{F} \cdot \sigma$ est nul en dehors des éléments unipotents réguliers de $G^{F} \cdot \sigma$ quand $\sigma$ est unipotent (resp. en dehors des éléments pseudo-unipotents réguliers de $G^{F} \cdot \sigma$, i.e. des conjugués sous $G$ d'éléments réguliers de $U \cdot \sigma$, quand $\sigma$ est semi-simple) (proposition 7.2).

Les représentations de Gelfand-Graev peuvent être utilisées pour calculer la valeur moyenne des caractères irréductibles de $G^{F} \cdot\langle\sigma\rangle$ sur un ensemble de 
classes de $G^{F}$-conjugaison d'éléments unipotents (resp. pseudo-unipotents) réguliers de $G^{F} \cdot \sigma$ si $\sigma$ est unipotent (resp. semi-simple) (théorème 8.4). Quand $\sigma$ est semi-simple et quand la caractéristique est bonne pour $\left(G^{\sigma}\right)^{0}$, on peut alors obtenir les valeurs exactes des caractères irréductibles de $G^{F} \cdot\langle\sigma\rangle$ sur les classes de $G^{F}$-conjugaison d'éléments pseudo-unipotents réguliers de $G^{F} \cdot \sigma$.

\section{Résultats généraux sur les groupes réductifs non connexes}

Soit $\widetilde{G}$ un groupe réductif non connexe. On note $G$ la composante neutre de $\widetilde{G}$. Nous utiliserons les définitions suivantes, introduites dans [4], qui généralisent les notions de tore et de sous-groupe de Borel :

DÉFINITION 2.1. - Un quasi-borel de $\widetilde{G}$ est le normalisateur dans $\widetilde{G}$ d'un sous-groupe de Borel de $G$. Un quasi-tore de $\widetilde{G}$ est le normalisateur dans $\widetilde{G}$ d'un couple $T \subset B$ formé d'un tore maximal et d'un sous-groupe de Borel de $G$.

On note $\mathbb{F}_{q}$ un corps fini de caractéristique $p$ à $q$ éléments ( $q$ étant une puissance de $p$ ). Désormais et jusqu'à la fin de l'article, on considère un groupe réductif non connexe $\widetilde{G}$ de la forme

$$
\widetilde{G}=G \cdot\langle\sigma\rangle,
$$

où $G$ est un groupe réductif connexe sur la clôture algébrique $\overline{\mathbb{F}}_{q}$ de $\mathbb{F}_{q}$ défini sur $\mathbb{F}_{q}$ (on note $F$ l'endomorphisme de Frobenius correspondant) et $\sigma$ un automorphisme de $G$. On dit que $\sigma$ est quasi-semi-simple s'il fixe un couple $(T, B)$ formé par un tore maximal $T$ de $G$ inclus dans un sous-groupe de Borel $B$ de $G$.

Le théorème suivant explicite les relations qui existent alors entre $G$ et le groupe $G^{\sigma}$ des points de $G$ fixes par $\sigma$.

THÉORÈme 2.2 (voir [4], th. 1.8). - (i) $G^{\sigma}$ est un groupe réductif.

(ii) Soit $T$ un tore maximal $\sigma$-stable inclus dans un sous-groupe de Borel $\sigma$ stable $B$ de $G$. Soit $\Phi$ l'ensemble des racines relativement à $(G, T)$. Si $\alpha \in \Phi$, si $\lambda \mapsto x_{\alpha}(\lambda)$ est le sous-groupe à un paramètre correspondant, et si $i$ est l'ordre de la $\sigma$-orbite de $\alpha$, on définit $C_{\sigma, \alpha} \in \overline{\mathbb{F}}_{q}^{\times}$par

$$
\sigma^{i}\left(x_{\alpha}(\lambda)\right)=x_{\alpha}\left(C_{\sigma, \alpha} \lambda\right) .
$$

Alors, il existe une surjection naturelle de l'ensemble des orbites sous $\sigma$ vérifiant la condition

$$
C_{\sigma, \alpha}= \pm 1
$$

où -1 n'est autorisé que s'il existe deux racines de l'orbite dont la somme est une racine, sur l'ensemble des racines de $\left(G^{\sigma}\right)^{0}$ relativement à $\left(T^{\sigma}\right)^{0}$. Cette surjection est bijective et tous les $C_{\sigma, \alpha}$ valent 1 , sauf si le diagramme de Dynkin de $G$ possède $k$ composantes de type $A_{2 n}$ permutées circulairement par $\sigma$,

TOME $132-2004-\mathrm{N}^{\mathrm{O}} 2$ 
où $\sigma^{k}$ agit par retournement de chacune de ces composantes. Alors, pour toute racine $\alpha$ telle que $\alpha+\sigma^{k}(\alpha)$ soit une racine, les orbites de $\alpha$ et de $\alpha+\sigma^{k}(\alpha)$ ont même image et $C_{\sigma, \alpha}=C_{\sigma, \alpha+\sigma^{k}(\alpha)}$.

On dit que $\sigma$ est quasi-central s'il est quasi-semi-simple et si l'on a $\operatorname{dim}\left(G^{\sigma}\right)>\operatorname{dim}\left(G^{\sigma^{\prime}}\right)$ pour tout automorphisme quasi-semi-simple $\sigma^{\prime}$ tel que $\sigma^{\prime}=\sigma \circ \operatorname{ad}(g)$, où on note $\operatorname{ad}(g)$ la conjugaison par $g$ pour tout $g \in G$.

ThÉorème 2.3 (voir [4, th. $1.15,1.29,1.33$ et 1.36$]$ et [5, prop. 2.1])

(i) Si $\sigma$ est quasi-semi-simple, alors $\sigma$ est quasi-central si et seulement si, pour tout couple $(T, B)$ où $T$ est un tore maximal $\sigma$-stable inclus dans un sousgroupe de Borel $\sigma$-stable $B$ de $G$, toute racine simple relativement à $(T, B)$ vérifie la condition $\left.{ }^{*}\right)$ du théorème 2.2 .

(ii) Si $\sigma$ est quasi-central, alors on a $G^{\sigma}=\left(G^{\sigma}\right)^{0} \cdot \mathrm{Z}\left(G^{\sigma}\right)$.

(iii) Si $\sigma$ est quasi-central unipotent, alors $G^{\sigma}$ est connexe et si $T$ est un tore maximal $\sigma$-stable inclus dans un sous-groupe de Borel $\sigma$-stable, $T^{\sigma}$ est connexe et on a $T=T^{\sigma} \times \mathcal{L}(T)$, où $\mathcal{L}: T \rightarrow T$ est définie par $t \mapsto t^{-1} \sigma(t)$.

(iv) Si $\sigma$ est quasi-central et rationnel, alors, il existe un couple $(T, B)$ formé par un tore maximal rationnel $\sigma$-stable $T$ inclus dans un sous-groupe de Borel rationnel $\sigma$-stable $B$ de $G$.

Notation 2.4. - Lorsque pour toute racine simple $\alpha$, on a l'égalité $C_{\sigma, \alpha}=1$, on dira que $\sigma$ vérifie la condition (RS).

REMARQUe 2.5. - Lorsque $\sigma$ est unipotent quasi-central, la condition (RS) est vérifiée.

Démonstration. - Comme $\sigma$ est quasi-central, on a $C_{\sigma, \alpha}= \pm 1$ pour toutes les racines $\alpha \in \Pi$ et tous les $C_{\sigma, \alpha}$ valent 1 , sauf si le diagramme de Dynkin de $\widetilde{G}$ possède $k$ composantes de type $A_{2 n}$ permutées circulairement par $\sigma$ où $\sigma^{k}$ agit par retournement de chacune de ces composantes. Mais, dans ce cas particulier, le cardinal de l'orbite de $\alpha$ est alors égal à $2 k$; or on a supposé $\sigma$ unipotent, donc l'ordre de $\sigma$ est une puissance de la caractéristique de $\overline{\mathbb{F}}_{q}$. Mais comme l'ordre de $\alpha$ divise l'ordre de $\sigma$, c'est donc que la caractéristique de $\overline{\mathbb{F}}_{q}$ vaut 2 . Ainsi, on a encore $C_{\sigma, \alpha}=1$.

On supposera par la suite que $\sigma$ est rationnel, quasi-central, unipotent ou semi-simple. On choisit un couple $T \subset B$ où $T$ est un tore maximal rationnel $\sigma$ stable inclus dans un sous-groupe de Borel rationnel $\sigma$-stable de $G$. On note $U$ le radical unipotent de $B$.

Soit $\Phi$ le système de racines de $(G, T)$. On choisit sur $\Phi$ l'ordre tel que

$$
U=\prod_{\alpha \in \Phi^{+}} U_{\alpha}
$$


et on note $\Pi$ la base de racines correspondante. Pour toute racine $\alpha \in \Phi$, on fixe un isomorphisme $x_{\alpha}: \overline{\mathbb{F}}_{q}^{+} \rightarrow U_{\alpha}$ et on définit $c_{\alpha}$ par $\sigma\left(x_{\alpha}(a)\right)=x_{\sigma(\alpha)}\left(c_{\alpha} a\right)$ pour tout $a \in \overline{\mathbb{F}}_{q}^{+}$, on note $o(\alpha)=\min \left\{i ; \sigma^{i}(\alpha)=\alpha\right\}$, le cardinal de la $\sigma$ orbite de $\alpha$, et on retrouve le coefficient $C_{\sigma, \alpha}$ du théorème 2.2 , en effet, on a $C_{\sigma, \alpha}=\prod_{i=0}^{o(\alpha)-1} c_{\sigma^{i}(\alpha)}$. Enfin, on pose

$$
U^{*}=\prod_{\alpha \in \Phi^{+}-\Pi} U_{\alpha} \quad \text { et } \quad \widetilde{U}=U / U^{*} .
$$

\section{3. Éléments réguliers}

Comme nous le verrons dans la section 7 , les propriétés des représentations de Gelfand-Graev impliquent ceux des éléments réguliers. Dans cette section, nous donnons leur définition et quelques unes de leurs propriétés. Nous allons nous intéresser particulièrement aux éléments réguliers de $G \cdot \sigma$ qui sont unipotents lorsque $\sigma$ est quasi-central unipotent et pseudo-unipotents lorsque $\sigma$ est quasi-central semi-simple (lorsque $\sigma$ est semi-simple, on appelle élément pseudo-unipotent de $G \cdot \sigma$ tout conjugué sous $G$ d'un élément de $U \cdot \sigma)$.

DÉfinition 3.1. - Soit y un élément d'un groupe réductif non connexe $H$. Alors $y$ est dit régulier dans $H$ si la dimension de son centralisateur dans $H$ est minimale dans la composante connexe $H^{0} \cdot y$, où $H^{0}$ est la composante neutre de $H$.

\section{1. Éléments unipotents réguliers de $G \cdot \sigma$ lorsque $\sigma$ est unipotent}

On suppose que $\sigma$ est unipotent quasi-central rationnel.

Proposition 3.2. - Les éléments unipotents réguliers existent dans $G \cdot \sigma$ et ils sont tous conjugués sous $G$.

Démonstration. — Voir [7, cor. I.4.8].

Proposition 3.3. - Soit $u \cdot \sigma \in G \cdot \sigma$ un élément unipotent; les conditions suivantes sont équivalentes :

(a) $u \cdot \sigma$ est régulier;

(b) $\operatorname{dim}\left(\mathrm{C}_{G}(u \cdot \sigma)\right)=\operatorname{rg}\left(G^{\sigma}\right)$;

(c) $\operatorname{dim}\left(\mathcal{B}_{u \cdot \sigma}^{G}\right)=0$, où $\mathcal{B}_{u \cdot \sigma}^{G}$ est la variété des sous-groupes de Borel de $G$ normalisés par $u \cdot \sigma$;

(d) $\left|\mathcal{B}_{u \cdot \sigma}^{G}\right|=1$;

(e) $u \cdot \sigma$ est conjugué sous $G$ à un élément de la forme $\prod_{i=1}^{r} x_{\alpha_{i}}(1) u^{*} \cdot \sigma$, où $\alpha_{1}, \ldots, \alpha_{r}$ est un système de représentants des $\sigma$-orbites dans $\Pi$ et $u^{*}$ est un élément quelconque de $U^{*}$.

Démonstration. — Voir [7, prop. II.10.2].

TOME $132-2004-\mathrm{N}^{\mathrm{O}} 2$ 
ThÉorème 3.4. - Soit $u=\prod_{\alpha \in \Phi^{+}} x_{\alpha}\left(\lambda_{\alpha}\right)$ un élément de U. Alors $u \cdot \sigma$ est unipotent et il est régulier si et seulement si pour toute racine $\alpha \in \Pi$,

$$
\sum_{n=0}^{o(\alpha)-1} c_{\sigma^{n}(\alpha)} c_{\sigma^{n+1}(\alpha)} \cdots c_{\sigma^{o(\alpha)-1}(\alpha)} \lambda_{\sigma^{n}(\alpha)} \neq 0 .
$$

Démonstration. - Soient $u$ un élément de $U$ et $k$ l'ordre de $\sigma$. Alors

$$
(u \cdot \sigma)^{k}=u \sigma(u) \cdots \sigma^{k-1}(u) .
$$

Or $U$ est un sous-groupe $\sigma$-stable de $G$, donc $u \sigma(u) \cdots \sigma^{k-1}(u)$ est un élément de $U$ et est unipotent ; cela suffit pour conclure que $u \cdot \sigma$ l'est aussi car on est en caractéristique $p$.

On peut ainsi appliquer à $u \cdot \sigma$ la proposition 3.3 : l'élément $u \cdot \sigma$ est régulier unipotent si et seulement si il est conjugué sous $G$ à un élément de la forme

$$
u_{0} \cdot \sigma=\prod_{i=1}^{r} x_{\alpha_{i}}(1) u^{*} \cdot \sigma
$$

où $\alpha_{1}, \ldots, \alpha_{r}$ est un système de représentants des $\sigma$-orbites dans $\Pi$ et $u^{*}$ est un élément quelconque de $U^{*}$.

On peut tout d'abord remarquer que $u \cdot \sigma$ et $u_{0} \cdot \sigma$ sont conjugués sous $G$ si et seulement si ils sont conjugués sous $T^{\sigma} U$. Soit en effet $x \in G$ tel que $u \cdot \sigma={ }^{x}\left(u_{0} \cdot \sigma\right)$; alors ${ }^{x}\left(u_{0} \cdot \sigma\right) \in \mathrm{N}_{\widetilde{G}}(B)$, donc $u_{0} \cdot \sigma$ normalise ${ }^{x^{-1}} B$, ce qui entraîne que $x \in B$ car $u_{0} \cdot \sigma$ est unipotent régulier. De plus, supposons trouvé $b=v t \in U T$ tel que $u \cdot \sigma={ }^{b}\left(u_{0} \cdot \sigma\right)$; alors $u^{-1} v^{t} u_{0}{ }^{t \sigma(t)^{-1}} \sigma(v)^{-1}=\sigma(t) t^{-1}$, donc $t \in T^{\sigma}$. Donc $u \cdot \sigma$ est conjugué à $u_{0} \cdot \sigma$ si et seulement si il existe $t \in T^{\sigma}$ et $v \in U$ tels que $u=v^{t} u_{0} \sigma(v)^{-1}$.

On note $p$ la projection de $U$ sur $\widetilde{U}=U / U^{*}$.

Lemme 3.5. - L'élément $u$ est $\sigma$-conjugué à un élément de la forme

$$
u_{0}=\prod_{i=1}^{r} x_{\alpha_{i}}(1) u^{*}, \quad \text { avec } u^{*} \in U^{*}
$$

si et seulement si $p(u)$ est $\sigma$-conjugué à $\tilde{u}_{0}=\prod_{i=1}^{r} x_{\alpha_{i}}(1) \in \widetilde{U}$.

Démonstration. - Pour tout $t \in T^{\sigma}$ on note $\Phi_{t}$ l'application de $U \times U$ dans $U$ telle que $\Phi_{t}:(x, y) \mapsto y^{t} x \sigma(y)^{-1}$. Soient $t \in T^{\sigma},(x, y) \in U \times U$ et $x^{\prime}, y^{\prime}$ quelconques dans $U^{*}$; alors on a

$$
\Phi_{t}\left(x x^{\prime}, y y^{\prime}\right)=y y^{\prime t}\left(x x^{\prime}\right) \sigma\left(y y^{\prime}\right)^{-1}=y^{t} x \sigma(y)^{-1} w^{\prime}
$$

avec

$$
w^{\prime}=\left[\left(\left[y^{\prime},{ }^{t} x\right] y^{\prime t} x^{\prime} \sigma\left(y^{\prime}\right)^{-1}\right), \sigma(y)^{-1}\right]\left(\left[y^{\prime},{ }^{t} x\right] y^{\prime t} x^{\prime} \sigma\left(y^{\prime}\right)^{-1}\right) \in U^{*} .
$$

BULletin DE LA SOCiÉtÉ MATHÉMATIQUE DE FRANCE 
Donc, pour tout $t \in T^{\sigma}$, l'application $\Phi_{t}$ passe au quotient ; soit $\widetilde{\Phi}_{t}: \widetilde{U} \times \widetilde{U} \rightarrow \widetilde{U}$ telle que

$$
\widetilde{\Phi}_{t}(\tilde{x}, \tilde{y})=\tilde{y}^{t} \tilde{x} \sigma(\tilde{y})^{-1}
$$

pour tout $(\tilde{x}, \tilde{y}) \in \widetilde{U} \times \widetilde{U}$. Alors on a $p\left(\Phi_{t}(x, y)\right)=\widetilde{\Phi}_{t}(p(x), p(y))$ pour tout couple $(x, y)$ dans $U \times U$.

Ainsi, $u$ est $\sigma$-conjugué à un élément de la forme

$$
u_{0}=\prod_{i=1}^{r} x_{\alpha_{i}}(1) u^{*}, \quad \text { avec } u^{*} \in U^{*},
$$

si et seulement si il existe $t \in T^{\sigma}$ et $v \in U$ tels que $u=\Phi_{t}\left(u_{0}, v\right)$, ce qui implique que $p(u)=\widetilde{\Phi}_{t}\left(p\left(u_{0}\right), p(v)\right)$. Réciproquement, si on pose $\tilde{u}_{0}=\prod_{i=1}^{r} x_{\alpha_{i}}(1)$ dans $U / U^{*}$ et si $p(u)=\widetilde{\Phi}_{t}\left(\tilde{u_{0}}, p(v)\right)$, alors $u=\Phi_{t}\left(u_{0}, v\right)$ pour un $u_{0}$ de la forme $u_{0}=\prod_{i=1}^{r} x_{\alpha_{i}}(1) u^{*}$. Donc, pour que $u$ soit $\sigma$-conjugué à un élément de la forme $u_{0}=\prod_{i=1}^{r} x_{\alpha_{i}}(1) u^{*}$, il faut et il suffit que $p(u)$ soit $\sigma$-conjugué à $\tilde{u}_{0}$.

Notons $o(i)$ le cardinal de la $\sigma$-orbite de $\alpha_{i}$. D'après le lemme ci-dessus, pour que $u \cdot \sigma=\prod_{\alpha \in \Phi^{+}} x_{\alpha}\left(\lambda_{\alpha}\right) \cdot \sigma$ soit unipotent régulier, il faut et il suffit qu'il existe $t \in T^{\sigma}$ et $v \in \widetilde{U}$ tels que si $\tilde{u}=\prod_{\alpha \in \Pi} x_{\alpha}\left(\lambda_{\alpha}\right)$, alors $\tilde{u}=v^{t} \tilde{u}_{0} \sigma(v)^{-1}$. On pose $v=\prod_{\alpha \in \Pi} x_{\alpha}\left(\ell_{\alpha}\right)$. Alors

$$
\begin{aligned}
\tilde{u}= & v^{t} \tilde{u}_{0} \sigma(v)^{-1} \\
& \Longleftrightarrow \forall i \in\{1, \ldots, r\}, \forall j \in\{0, \ldots, o(i)-1\} \\
& \quad \ell_{\sigma^{j}\left(\alpha_{i}\right)}+\alpha_{i}(t)-c_{\sigma^{j-1}\left(\alpha_{i}\right)} \ell_{\sigma^{j-1}\left(\alpha_{i}\right)}=\lambda_{\sigma^{j}\left(\alpha_{i}\right)} \\
& \Longleftrightarrow \forall i \in\{1, \ldots, r\}, \quad A_{i} L_{i}=B_{i}
\end{aligned}
$$

avec

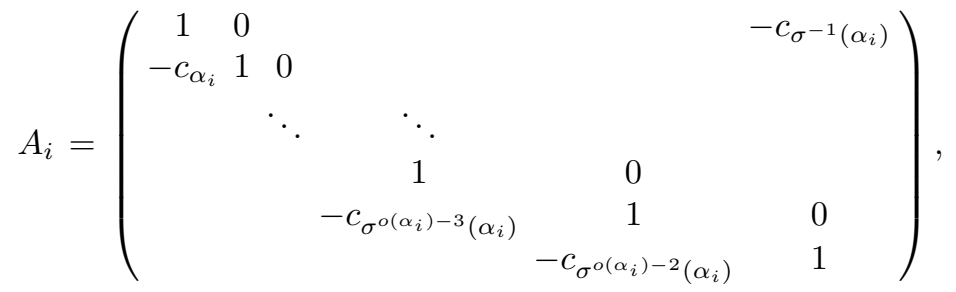

$$
\begin{aligned}
& B_{i}=\left(\begin{array}{c}
-\alpha_{i}(t)+\lambda_{\alpha_{i}} \\
\vdots \\
-\alpha_{i}(t)+\lambda_{\sigma^{o\left(\alpha_{i}\right)-1}\left(\alpha_{i}\right)}
\end{array}\right), \quad L_{i}=\left(\begin{array}{c}
\ell_{\alpha_{i}} \\
\vdots \\
\ell_{\sigma^{o\left(\alpha_{i}\right)-1}\left(\alpha_{i}\right)}
\end{array}\right)
\end{aligned}
$$

Soit $i \in\{1, \ldots, r\}$; alors, d'après la remarque 2.5 , le coefficient

$$
C_{\sigma, \alpha_{i}}=c_{\alpha_{i}} \cdots c_{\sigma^{o\left(\alpha_{i}\right)-1}}
$$

TOME $132-2004-\mathrm{N}^{\mathrm{O}} 2$ 
est égal à 1. Ainsi, $A_{i}$ est une matrice $o\left(\alpha_{i}\right) \times o\left(\alpha_{i}\right)$ de rang $o\left(\alpha_{i}\right)-1$ et $A_{i} L_{i}=B_{i}$ a une solution si et seulement si

$$
\begin{array}{r}
\left(c_{\alpha_{i}} \cdots c_{\sigma^{o\left(\alpha_{i}\right)-2}\left(\alpha_{i}\right)}+\cdots+c_{\sigma^{o\left(\alpha_{i}\right)-2}\left(\alpha_{i}\right)}+1\right) \alpha_{i}(t) \\
=c_{\alpha_{i}} \cdots c_{\sigma^{o\left(\alpha_{i}\right)-2}\left(\alpha_{i}\right)} \lambda_{\alpha_{i}}+\cdots+\lambda_{\sigma^{o\left(\alpha_{i}\right)-1}\left(\alpha_{i}\right)}
\end{array}
$$

Ainsi, on a montré que $u \cdot \sigma$ est unipotent régulier si et seulement s'il existe $t \in T^{\sigma}$ tel que $\left({ }^{*} i\right)$ soit vérifié pour tout $i \in\{1, \ldots, r\}$. Or un tel $t$ existe si et seulement si le coefficient de $\alpha_{i}(t)$ dans le membre de gauche de $\left({ }^{*} i\right)$ est non nul pour tout $i$, ce qui est vrai car pour tout $r$-uplet $\left(x_{1}, \ldots, x_{r}\right)$ de $\left(\overline{\mathbb{F}}_{q}^{\times}\right)^{r}$, il existe $t \in T$ tel que pour tout $i \in\{1, \ldots, r\}$, on ait $\alpha_{i}(t)=\cdots=\sigma^{o(i)-1}\left(\alpha_{i}\right)(t)=x_{i}$ (cf. [3, lemme 0.22$]$ ), et alors on a $t \in T^{\sigma}$.

Ainsi, $u \cdot \sigma$ est unipotent régulier si et seulement si pour tout $i \in\{1, \ldots, r\}$, on a

$$
c_{\alpha_{i}} \cdots c_{\sigma^{o\left(\alpha_{i}\right)-2}\left(\alpha_{i}\right)} \lambda_{\alpha_{i}}+\cdots+c_{\sigma^{o\left(\alpha_{i}\right)-2}\left(\alpha_{i}\right)} \lambda_{\sigma^{o\left(\alpha_{i}\right)-2}\left(\alpha_{i}\right)}+\lambda_{\sigma^{o\left(\alpha_{i}\right)-1}\left(\alpha_{i}\right)} \neq 0 .
$$

On retrouve alors l'équation du théorème 3.4 en multipliant l'équation ci-dessus par $c_{\sigma^{o(i)-1}(\alpha)}$.

THÉORÈme 3.6. - Si l est le rang semi-simple de $G^{\sigma}$, le nombre d'éléments unipotents réguliers rationnels dans $G \cdot \sigma$ est égal à

$$
\frac{\left|G^{F}\right|}{\left|\left(Z\left(G^{\sigma}\right)^{0}\right)^{F}\right| \times q^{\ell}} .
$$

Démonstration. — La preuve du théorème 3.6 résulte de plusieurs lemmes.

LEMME 3.7. — Dans tout quasi-borel rationnel, il y a le même nombre d'éléments unipotents réguliers rationnels de $G \cdot \sigma$.

Démonstration. - Le résultat est clair car le conjugué sous $G^{F}$ d'un unipotent régulier rationnel l'est aussi et deux quasi-borel rationnels sont conjugués sous $G^{F}$. Donc la conjugaison envoie bijectivement les éléments unipotents réguliers rationnels de l'un sur ceux de l'autre.

LEMME 3.8. - Tout élément unipotent régulier rationnel est dans un quasiborel rationnel.

Démonstration. - Soit $x$ un élément unipotent régulier rationnel. Soit $B^{\prime}$ l'unique sous-groupe de Borel de $G$ normalisé par $x$. On a $F\left({ }^{x} B^{\prime}\right)={ }^{x} F\left(B^{\prime}\right)$ car $x$ est rationnel et $F\left({ }^{x} B^{\prime}\right)=F\left(B^{\prime}\right)$ car $x$ normalise $B^{\prime}$, donc $x$ normalise aussi $F\left(B^{\prime}\right)$. Mais comme $x$ normalise un unique sous-groupe de Borel de $G$, on a $F\left(B^{\prime}\right)=B^{\prime}$.

Un élément unipotent régulier rationnel de $G \cdot \sigma$ est dans un seul quasi-borel et celui-ci est rationnel. Donc le nombre d'éléments unipotents réguliers de $(G \cdot \sigma)^{F}$ est égal au nombre de sous-groupes de Borel rationnels de $G$ multiplié 
par le nombre de tels éléments dans l'intersection de $G \cdot \sigma$ et du quasi-borel rationnel $\mathrm{N}_{\widetilde{G}}(B)$.

LEMME 3.9. - L'ensemble des éléments réguliers unipotents de $\mathrm{N}_{\widetilde{G}}(B) \cap G \cdot \sigma$ est de la forme $\mathcal{U}_{B}=\left\{b u \sigma(b)^{-1} \cdot \sigma ; b \in B\right\}$ où $u \cdot \sigma$ est un élément unipotent régulier fixé de $\mathrm{N}_{\widetilde{G}}(B)$.

Démonstration. - Soit $u \cdot \sigma$ un élément unipotent régulier rationnel de $\mathrm{N}_{\widetilde{G}}(B)$. Soit $x \in G$ tel que ${ }^{x}(u \cdot \sigma) \in \mathrm{N}_{\widetilde{G}}(B)$. Alors ${ }^{x u \sigma x^{-1}} B=B$, ce qui entraîne que $u \cdot \sigma\left(x^{-1} B\right)=x^{-1} B$. Or si $x \notin B$, on a ${ }^{x^{-1}} B \neq B$ et ${ }^{x^{-1}} B, B$ sont tous les deux normalisés par $u \cdot \sigma$ ce qui est impossible car $u \cdot \sigma$ est régulier. Ainsi on a montré que tout $x \in G$ tel que ${ }^{x}(u \cdot \sigma) \in \mathrm{N}_{\widetilde{G}}(B)$ appartient à $B$. Par ailleurs dans $G \cdot \sigma$, il y a une unique classe unipotente régulière. Donc les éléments unipotents réguliers de $\mathrm{N}_{\widetilde{G}}(B) \cap G \cdot \sigma$ sont les conjugués sous $B$ de $u \cdot \sigma$. Si on note $\mathcal{U}_{B}$ l'ensemble de ces éléments, on a $\mathcal{U}_{B}=\left\{b u \sigma(b)^{-1} \cdot \sigma ; b \in B\right\}$.

Calculons le nombre d'éléments unipotents réguliers rationnels dans l'intersection de $G \cdot \sigma$ et du quasi-borel $\mathrm{N}_{\widetilde{G}}(B)$.

Soit $u_{0} \cdot \sigma$ un élément de $\mathrm{N}_{\widetilde{G}}(B)$ unipotent régulier et rationnel. Alors le nombre cherché est égal au nombre de conjugués de $u_{0} \cdot \sigma$ sous $B$ qui sont rationnels, c'est-à-dire au nombre de $\sigma$-conjugués de $u_{0}$ sous $B$ qui sont rationnels. On pose $\widetilde{B}=B / U^{*}$. Soit $\phi: B \rightarrow B$ définie par $b \mapsto b u_{0} \sigma(b)^{-1}$. Soient $b$ et $b^{\prime}$ dans $B$ tels qu'il existe $u \in U^{*}$ tel que $b^{\prime}=b u$. Alors on a $\phi\left(b^{\prime}\right)=\phi(b) v$ avec $v \in U^{*}$ (car $B$ normalise $U^{*}$ ) et $\phi$ passe au quotient. Par ailleurs, d'après le lemme 3.5 et la partie (e) de la proposition 3.3 , le fait qu'un élément $u \cdot \sigma$ où $u \in B$ est unipotent régulier ne dépend que de l'image de $u$ dans $U / U^{*}$ et tout élément rationnel de $\widetilde{B}$ a un représentant rationnel dans $B$. Cela montre donc que le nombre cherché est égal au nombre de $\sigma$-conjugués rationnels de $\tilde{u}_{0}$ sous $\widetilde{B}$ (où on note $\tilde{u}_{0}$ l'image de $u_{0}$ dans $\widetilde{B}$ ) multiplié par $\left|U^{* F}\right|$. On note $b \cdot \tilde{u}_{0}$ l'action de $\sigma$-conjugaison de $b \in \widetilde{B}$ sur $\tilde{u}_{0}$. Soit

$$
\mathcal{O}_{B}=\left\{b \cdot \tilde{u}_{0} ; b \in \widetilde{B}\right\} .
$$

Alors $\mathcal{O}_{B}$ est $F$-stable car $\tilde{u}_{0}$ est rationnel.

Proposition 3.10 (voir [3, prop. 3.21]). - (i) Soit $g \in \widetilde{B}$. Alors $g \cdot \tilde{u}_{0} \in \mathcal{O}_{\mathrm{B}}^{\mathrm{F}}$ si et seulement si $g^{-1} F g \in \operatorname{Stab}\left(\tilde{u}_{0}\right)$ où $\operatorname{Stab}\left(\tilde{u}_{0}\right)=\left\{g \in \widetilde{B} ; g \cdot \tilde{u}_{0}=\tilde{u}_{0}\right\}$.

(ii) Il y a une application bien définie qui envoie la $\widetilde{B}^{F}$-orbite de $g \cdot \tilde{u}_{0} \in \mathcal{O}_{B}^{F}$ sur la classe de F-conjugaison de l'image de $g^{-1 F} g$ dans $\operatorname{Stab}\left(\tilde{u}_{0}\right) / \operatorname{Stab}\left(\tilde{u}_{0}\right)^{0}$; c'est une bijection et le stabilisateur de $g \cdot \tilde{u}_{0}$ dans $\widetilde{B}^{F}$ est isomorphe au F-centralisateur de $g^{-1 F} g$ dans $\operatorname{Stab}\left(\tilde{u}_{0}\right)$.

D'après le (i), si $\operatorname{Stab}\left(\tilde{u}_{0}\right)$ est abélien, le nombre d'éléments est le même dans toutes les $\widetilde{B}^{F}$-orbites et vaut $\left|\widetilde{B}^{F} / \operatorname{Stab}\left(\tilde{u}_{0}\right)^{F}\right|$. Cela entraîne avec le (ii)

TOME $132-2004-\mathrm{N}^{\mathrm{O}} 2$ 
que le nombre de $\sigma$-conjugués de $\tilde{u}_{0}$ dans $\widetilde{B}^{F}$ est égal au nombre de $F$-classes de conjugaison de $\operatorname{Stab}\left(\tilde{u}_{0}\right) / \operatorname{Stab}\left(\tilde{u}_{0}\right)^{0}$ multiplié par $\left|\widetilde{B}^{F} / \operatorname{Stab}\left(\tilde{u}_{0}\right)^{F}\right|$.

On note $\mathrm{H}^{1}(F, H)$ l'ensemble des classes de $F$-conjugaison d'un groupe algébrique $H$ défini sur $\mathbb{F}_{q}$. On a

$$
\mathrm{H}^{1}(F, H)=\mathrm{H}^{1}\left(F, H / H^{0}\right)
$$

et si $H / H^{0}$ est abélien,

$$
\left|\mathrm{H}^{1}\left(F, H / H^{0}\right)\right|=\left|H^{F} /\left(H^{0}\right)^{F}\right|
$$

Lemme 3.11. - On a $\operatorname{Stab}\left(\tilde{u}_{0}\right)=\mathrm{Z}(G)^{\sigma} \times \widetilde{U}^{\sigma}$.

En utilisant le lemme ci-dessus, on obtient l'expression suivante du nombre d'éléments réguliers unipotents de $(G \cdot \sigma)^{F}$ :

$$
\begin{gathered}
\left(\left|G^{F}\right| /\left|B^{F}\right|\right) \times\left|\left(U^{*}\right)^{F}\right| \times\left|\mathrm{H}^{1}\left(F, \operatorname{Stab}\left(\tilde{u}_{0}\right) / \operatorname{Stab}\left(\tilde{u}_{0}\right)^{0}\right)\right| \times\left(\left|\widetilde{B}^{F}\right| /\left|\operatorname{Stab}\left(\tilde{u}_{0}\right)^{F}\right|\right) \\
=\left|G^{F}\right| /\left(\left|\left(U^{*}\right)^{F}\right| \times\left|\widetilde{B}^{F}\right|\right) \times\left|\left(U^{*}\right)^{F}\right| \times\left|\operatorname{Stab}\left(\tilde{u}_{0}\right)^{F} /\left(\operatorname{Stab}\left(\tilde{u}_{0}\right)^{0}\right)^{F}\right| \\
\times\left(\left|\widetilde{B}^{F}\right| /\left|\operatorname{Stab}\left(\tilde{u}_{0}\right)^{F}\right|\right) \\
=\left|G^{F}\right| /\left|\left(\operatorname{Stab}\left(\tilde{u}_{0}\right)^{0}\right)^{F}\right|=\left|G^{F}\right| /\left(\left|\left(\mathrm{Z}\left(G^{\sigma}\right)^{0}\right)^{F}\right| \times\left|\left(\widetilde{U}^{\sigma}\right)^{F}\right|\right) .
\end{gathered}
$$

Or $\widetilde{U}^{\sigma}=\left(\prod_{\alpha \in \Pi} U_{\alpha}\right)^{\sigma}$ est isomorphe à $\prod_{\alpha \in \Pi / \sigma} U_{\alpha}$ car les $C_{\sigma, \alpha}$ valent 1 (remarque 2.5); donc $\left|\left(\left(\prod_{\alpha \in \Pi} U_{\alpha}\right)^{F}\right)^{\sigma}\right|=q^{\ell}$, où $q$ est le rang semi-simple de $\left(G^{\sigma}\right)^{0}=G^{\sigma}$. Pour terminer la démonstration du théorème, il reste à démontrer le lemme 3.11 :

Démonstration. - Par définition, $\operatorname{Stab}\left(\tilde{u}_{0}\right)=\left\{b \in \widetilde{B} ; b \cdot \tilde{u}_{0}=\tilde{u}_{0}\right\}$. Soient $\left\{\alpha_{1}, \ldots, \alpha_{r}\right\}$ un ensemble de représentants des $\sigma$-orbites de $\Pi$. Pour tout $i \in\{1, \ldots, r\}$, on note $O(i)$ la $\sigma$-orbite de $\alpha_{i}, o(i)$ le cardinal de $O(i)$ et $U(i)=\prod_{\alpha \in O(i)} U_{\alpha} ;$ on pose enfin

$$
\tilde{u}_{0}=\prod_{i=1}^{r} \prod_{\alpha \in O(i)} x_{\alpha}\left(k_{\alpha}\right) \quad \text { et } \quad u=\prod_{i=1}^{r} \prod_{\alpha \in O(i)} x_{\alpha}\left(\ell_{\alpha}\right)
$$

BULLETIN DE LA SOCIÉTÉ MATHÉMATIQUe DE FRANCE 
Nous allons expliciter $\operatorname{Stab}\left(\tilde{u}_{0}\right)$ dans les calculs qui suivent. Soit $b=u t \in \widetilde{B}$ :

$$
\begin{aligned}
& (u t) \tilde{u}_{0} \sigma(u t)^{-1}=\tilde{u}_{0} \\
& \Longleftrightarrow\left({ }^{t} \tilde{u}_{0}\right)^{-1} u^{-1} \tilde{u}_{0} \sigma(u)=t \sigma(t)^{-1} \\
& \Longleftrightarrow t \in T^{\sigma} \text { et } u^{t} \tilde{u}_{0} \sigma(u)^{-1} \tilde{u}_{0}^{-1}=1 \\
& \left(\operatorname{car}\left({ }^{t} \tilde{u}_{0}\right)^{-1} u^{-1} \tilde{u}_{0} \sigma(u) \in \widetilde{U} \text { et } t \sigma(t)^{-1} \in T\right) \\
& \Longleftrightarrow t \in T^{\sigma} \text { et } \\
& \prod_{i=1}^{r} \prod_{\alpha \in O(i)} x_{\alpha}\left(\ell_{\alpha}\right)^{t}\left(\prod_{\alpha \in O(i)} x_{\alpha}\left(k_{\alpha}\right)\right) \\
& \times \sigma\left(\prod_{\alpha \in O(i)} x_{\alpha}\left(\ell_{\alpha}\right)\right)^{-1}\left(\prod_{\alpha \in O(i)} x_{\alpha}\left(k_{\alpha}\right)\right)^{-1}=1 \\
& \Longleftrightarrow t \in T^{\sigma} \text { et } \\
& \prod_{i=1}^{r} \prod_{\alpha \in O(i)} x_{\alpha}\left(\ell_{\alpha}\right) \prod_{\alpha \in O(i)} x_{\alpha}\left(\alpha(t) k_{\alpha}\right) \\
& \left(\prod_{\alpha \in O(i)} x_{\sigma(\alpha)}\left(c_{\alpha} \ell_{\alpha}\right)\right)^{-1}\left(\prod_{\alpha \in O(i)} x_{\alpha}\left(k_{\alpha}\right)\right)^{-1}=1 \\
& \Longleftrightarrow t \in T^{\sigma} \text { et } \prod_{i=1}^{r} \prod_{\alpha \in O(i)} x_{\alpha}\left(\ell_{\alpha}+\alpha(t) k_{\alpha}-c_{\sigma^{-1} \alpha} \ell_{\sigma^{-1} \alpha}-k_{\alpha}\right)=1 \\
& \Longleftrightarrow t \in T^{\sigma} \text { et } \forall i \in\{1, \ldots, r\}, \forall \alpha \in O(i) \text {, } \\
& \ell_{\alpha}+\alpha(t) k_{\alpha}-c_{\sigma^{-1} \alpha} \ell_{\sigma^{-1} \alpha}-k_{\alpha}=0 .
\end{aligned}
$$

On obtient le système d'équations suivant pour tout $i \in\{1, \ldots, r\}$

$$
\left\{\begin{array}{l}
\ell_{\alpha_{i}}-c_{\sigma^{o\left(\alpha_{i}\right)-1}\left(\alpha_{i}\right)} \ell_{\sigma^{o\left(\alpha_{i}\right)-1}\left(\alpha_{i}\right)}=\left(1-\alpha_{i}(t)\right) k_{\alpha_{i}} \\
\ell_{\sigma\left(\alpha_{i}\right)}-c_{\alpha_{i}} \ell_{\alpha_{i}}=\left(1-\alpha_{i}(t)\right) k_{\sigma\left(\alpha_{i}\right)} \\
\cdots \\
\ell_{\sigma^{o\left(\alpha_{i}\right)-1}\left(\alpha_{i}\right)}-c_{\sigma^{o\left(\alpha_{i}\right)-2}\left(\alpha_{i}\right)} \ell_{\sigma^{o\left(\alpha_{i}\right)-2}\left(\alpha_{i}\right)}=\left(1-\alpha_{i}(t)\right) k_{\sigma^{o\left(\alpha_{i}\right)-1}\left(\alpha_{i}\right)}
\end{array}\right.
$$

(car $t \in T^{\sigma}$ donc $\alpha(t)=\alpha_{i}(t)$ pour tout $\alpha \in O(i)$ ).

Or $C_{\sigma, \alpha}=1$ (remarque 2.5), donc le système (I) est de rang $o\left(\alpha_{i}\right)-1$ et il existe des solutions si et seulement si

$\left(c_{\alpha_{i}} \cdots c_{\sigma^{o\left(\alpha_{i}\right)-2}\left(\alpha_{i}\right)} k_{\alpha_{i}}+\cdots+c_{\sigma^{o\left(\alpha_{i}\right)-2}\left(\alpha_{i}\right)} k_{\sigma^{o\left(\alpha_{i}\right)-2}}+k_{\sigma^{o\left(\alpha_{i}\right)-1}}\right)\left(1-\alpha_{i}(t)\right)=0$.

D'après le théorème 3.4 , l'équation ci-dessus entraîne que $t \in \operatorname{Ker}\left(\alpha_{i}\right)$ et que

$$
\left\{\begin{array}{l}
\ell_{\sigma\left(\alpha_{i}\right)}=c_{\alpha_{i}} \ell_{\alpha_{i}}, \\
\cdots \\
\ell_{\sigma^{o\left(\alpha_{i}\right)-1}\left(\alpha_{i}\right)}=c_{\alpha_{i}} \cdots c_{\sigma^{o\left(\alpha_{i}\right)-2}\left(\alpha_{i}\right)} \ell_{\alpha_{i}} .
\end{array}\right.
$$

TOME $132-2004-\mathrm{N}^{\mathrm{O}} 2$ 
Donc $t \in \bigcap_{i=1}^{r} \operatorname{Ker} \alpha_{i} \cap T^{\sigma}=\mathrm{Z}(G)^{\sigma}$ et si on note $u=\prod_{i=1}^{r} u_{i}$ avec $u_{i} \in U(i)$, alors on a $u_{i} \in U(i)^{\sigma}$ pour tout $i \in\{1, \ldots, r\}$.

On démontre facilement que réciproquement

$$
\mathrm{Z}(G)^{\sigma} \times\left(\prod_{i=1}^{r} U(i)^{\sigma}\right) \subset \operatorname{Stab}\left(\tilde{u}_{0}\right) .
$$

D'où l'égalité. Ce qui termine les démonstrations du lemme 3.11 et du théorème 3.6 .

3.2. Éléments pseudo-unipotents réguliers de $G \cdot \sigma$ lorsque $\sigma$ est semi-simple. - On suppose que $\sigma$ est semi-simple quasi-central rationnel.

On rappelle que l'on appelle éléments pseudo-unipotents de $G \cdot \sigma$ les conjugués sous $G$ des éléments de $U \cdot \sigma$. Avant d'étudier les éléments pseudounipotents réguliers de $G \cdot \sigma$, on étudie les éléments réguliers de $U \cdot \sigma$.

Proposition 3.12. - Tout élément de $U \cdot \sigma$ est conjugué sous $U$ à un élément de $U^{\sigma} \cdot \sigma$.

Démonstration. - Lemme 3.13. - Tout élément semi-simple $v \cdot \sigma$ de $U \cdot \sigma$ est conjugué sous $U$ à $\sigma$.

Démonstration. - Selon [8, prop. 7.6], tout automorphisme semi-simple d'un groupe résoluble stabilise un tore maximal de ce groupe. Or tout élément semisimple $v \cdot \sigma$ avec $v \in U$ induit un automorphisme semi-simple de $B$ et donc stabilise un tore maximal ${ }^{x} T$ de $B$, conjugué de $T$ par un élément $x$ de $U$. Ainsi, $x^{-1}(v \cdot \sigma) x$ est un élément de $\mathrm{N}_{\widetilde{G}}(T) \cap U \cdot \sigma=\{\sigma\}$, ce qui montre directement que $v \cdot \sigma$ est conjugué à $\sigma$ sous $U$.

Soit $x \cdot \sigma \in U \cdot \sigma$; on note $x \cdot \sigma=u s$ la décomposition de Jordan de $x \cdot \sigma$. Alors $u \in U$ car $\sigma$ est semi-simple et $s$ est de la forme $v \cdot \sigma$ avec $v \in U$. On applique à $s=v \cdot \sigma$ le lemme précédent : il existe $w \in U$ tel que $x \cdot \sigma=w\left(w^{-1} u\right) \sigma w^{-1}$ et ${ }^{w^{-1}} u \in U^{\sigma}$ car $u$ commute à $s$; donc ${ }^{w^{-1}} u$ commute à ${ }^{w^{-1}} s=\sigma$.

Corollaire 3.14. - Un élément $u \cdot \sigma \in U \cdot \sigma$ est régulier si et seulement si il est conjugué sous $U$ à un élément $v \cdot \sigma \in U^{\sigma} \cdot \sigma$ tel que $v$ soit régulier dans $G^{\sigma}$.

Démonstration. - Selon la proposition précédente, $u \cdot \sigma \in U \cdot \sigma$ est conjugué sous $U$ à un élément $v \cdot \sigma \in U^{\sigma} \cdot \sigma$. Par unicité de la décomposition de Jordan de $v \cdot \sigma$, on a $\mathrm{C}_{G}(v \cdot \sigma)=\mathrm{C}_{G^{\sigma}}(v)$ et la dimension de $\mathrm{C}_{G}(v \cdot \sigma)$ est minimale si et seulement si la dimension de $\mathrm{C}_{G^{\sigma}}(v)$ l'est.

Proposition 3.15. - Il existe une unique G-classe de conjugaison pseudounipotente régulière dans $G \cdot \sigma$. 
Démonstration. - Par le corollaire 3.14, toute classe pseudo-unipotente régulière possède un représentant $v \cdot \sigma$ dans $U^{\sigma} \cdot \sigma$ avec $v$ régulier dans $G^{\sigma}$ et $v$ dans $U^{\sigma} \subset\left(G^{\sigma}\right)^{0}$. Comme tous les éléments unipotents réguliers de $\left(G^{\sigma}\right)^{0}$ sont conjugués dans $\left(G^{\sigma}\right)^{0}$ (cf. [3, prop. 14.16]), on obtient le résultat.

On a l'analogue de $3.3(\mathrm{~d})$ :

Proposition 3.16. - Un élément pseudo-unipotent régulier normalise un unique sous-groupe de Borel de G.

Démonstration. - On se ramène à un élément unipotent régulier de la forme $u \cdot \sigma$ avec $u \in U$. En utilisant le corollaire 3.14, on peut se ramener au cas où $u$ est $\sigma$-stable, régulier dans $\left(G^{\sigma}\right)^{0}$. Alors, si $u \cdot \sigma$ est contenu dans un quasi-borel, ses parties semi-simples et unipotentes le sont aussi, donc tout sous-groupe de Borel normalisé par $u \cdot \sigma$, l'est aussi par $u$ et par $\sigma$.

Supposons que $u$ normalise un sous-groupe de Borel $\sigma$-stable de $G$ autre que $B$; alors, comme deux sous-groupes de Borel $\sigma$-stables ont un tore $\sigma$ stable en commun, on peut se ramener au cas où il existe $n \in\left(\mathrm{N}_{G}(T)\right)^{\sigma}$ tel que $u \in U \cap{ }^{n} U$. On note $u=\prod_{\alpha \in \Phi^{+}} x_{\alpha}\left(k_{\alpha}\right)$. Soit $w \in W^{\sigma}$ tel que $n$ soit un représentant de $w$. Alors, pour toute racine $\alpha \in \Pi$ telle que $w(\alpha) \in \Phi^{-}$, on a $k_{\alpha}=0$. Si $w \neq 1$, alors, comme $w$ est $\sigma$-stable, il existe une $\sigma$-orbite $\mathcal{A}$ de $\Pi$ telle que pour toute racine $\alpha \in \mathcal{A}$, on ait $k_{\alpha}=0$. Or cela contredit le fait que $u$ soit régulier dans $\left(G^{\sigma}\right)^{0}$, d'où le résultat.

Le nombre d'éléments pseudo-unipotents réguliers rationnels dans $G \cdot \sigma$ est donné dans la remarque 8.11 .

\section{Caractères réguliers}

On garde les notations de la section 2 ; en particulier, on note $\Phi$ l'ensemble des racines associées à $(G, T)$ et $\Pi$ la base de racines associée à $B$. Alors $\left(B^{\sigma}\right)^{0}$ est un sous-groupe de Borel du groupe réductif connexe $\left(G^{\sigma}\right)^{0}$ de décomposition de Levi $\left(B^{\sigma}\right)^{0}=\left(T^{\sigma}\right)^{0} U^{\sigma}$. On note $\Phi^{\sigma}$ l'ensemble des racines associées à $\left(\left(G^{\sigma}\right)^{0},\left(T^{\sigma}\right)^{0}\right)$ et $\Pi / \sigma$ la base de racines associée à $\left(B^{\sigma}\right)^{0}$. De plus, $\tau$ sera l'action de $F$ sur les racines et si $\mathcal{O}$ est une $\tau$-orbite de $\Pi$, alors on note $U_{\mathcal{O}}$ l'image de $\prod_{\alpha \in \mathcal{O}} U_{\alpha}$ dans $U / U^{*}$ et $\Pi / \tau$ l'ensemble des $\tau$-orbites de $\Pi$. Pour toute racine $\alpha \in \Phi$, on fixe un isomorphisme $x_{\alpha}: \overline{\mathbb{F}}_{q}^{+} \rightarrow U_{\alpha}$ tel que $F\left(x_{\alpha}(a)\right)=x_{\tau(\alpha)}\left(a^{q}\right)$ et on définit comme dans la section précédente le coefficient $c_{\alpha}$ pour toute racine $\alpha \in \Phi$ par $\sigma\left(x_{\alpha}(a)\right)=x_{\sigma(\alpha)}\left(c_{\alpha} a\right)$. On fixe $\left\{\mathcal{O}_{1}, \ldots, \mathcal{O}_{s}\right\}$, un ensemble de représentants des $\sigma$-orbites de $\Pi / \tau$.

Dans le cas des groupes réductifs connexes, les représentations de GelfandGraev sont obtenues en induisant certains caractères linéaires irréductibles de $U^{F}$ appelés caractères réguliers. Nous généralisons la notion de caractères réguliers dans la définition 4.2 .

TOME $132-2004-\mathrm{N}^{\mathrm{O}} 2$ 
Notation 4.1. - Soit $\chi$ un caractère linéaire de $U^{F}$ tel que sa restriction à $\left(U^{*}\right)^{F}$ soit triviale. Alors $\chi$ se factorise à travers $\left(U / U^{*}\right)^{F} \simeq U^{F} / U^{* F}$. On identifie de cette façon $\chi$ à un caractère $\bar{\chi}$ de $U^{F} / U^{* F} \simeq \prod_{\mathcal{O} \in \Pi / \tau} U_{\mathcal{O}}^{F}$. Pour toute partie $F$-stable $I$ de $\Pi$,

(i) on note $U_{I}$ l'image de $\prod_{\alpha \in I} U_{\alpha}$ dans $U / U^{*}$;

(ii) on définit $\chi_{\mid U_{I}^{F}}$ comme la restriction de $\bar{\chi}$ à $U_{I}^{F}$.

DÉFINITION 4.2. - Un caractère multiplicatif $\tilde{\chi} \in \operatorname{Irr}\left(U^{F} \cdot\langle\sigma\rangle\right)$ est appelé caractère régulier si pour tout $u \in U^{F}$

$$
\widetilde{\chi}(u \cdot \sigma)=\chi(u) \tilde{\chi}(\sigma),
$$

où $\chi$ est un caractère $\sigma$-stable de $\operatorname{Irr}\left(U^{F}\right)$ qui vérifie les deux conditions suivantes :

(i) la restriction de $\chi$ à $\left(U^{*}\right)^{F}$ est triviale;

(ii) pour toute $\tau$-orbite $\mathcal{O}$ de $\Pi, \chi_{\mid U_{\mathcal{O}}^{F}}$ n'est pas triviale.

Sous ces conditions, on dit que $\chi$ est régulier et pour tout caractère régulier $\sigma$-stable $\chi$ de $U^{F}$; on note $\tilde{\chi}$ et on appelle extension normalisée de $\chi$ l'extension de $\chi$ à $U^{F} \cdot\langle\sigma\rangle$ telle que $\tilde{\chi}(\sigma)=1$.

Remarque 4.3. - Plaçons-nous dans le cas du groupe réductif connexe $\left(G^{\sigma}\right)^{0}$. Les caractères réguliers de $\left(U^{\sigma}\right)^{F}$ sont définis par les conditions (i) et (ii) de la définition précédente en remplaçant $U$ par $U^{\sigma}$ et $\Pi$ par $\Pi / \sigma$.

Proposition 4.4. - On suppose que la condition (RS) est vérifiée. Alors il existe une bijection entre les caractères réguliers de $\left(U^{\sigma}\right)^{F}$ et les caractères réguliers $\sigma$-stables de $U^{F}$.

Démonstration. — La démonstration repose sur une série de lemmes numérotés de 4.5 à 4.12 .

Lemme 4.5. - Pour toute racine $\alpha \in \Phi$ et pour tout entier $k$, on a

$$
c_{\tau^{k}(\alpha)}=\left(c_{\alpha}\right)^{q^{k}} .
$$

Démonstration. - Soient $\alpha \in \Phi$ et un entier $k$; alors, pour tout $\lambda \in \mathbb{F}_{q}^{+}$, on a

$$
\begin{aligned}
& \sigma\left(F^{k}\left(x_{\alpha}(\lambda)\right)\right)=\sigma\left(x_{\tau^{k}(\alpha)}\left(\lambda^{q^{k}}\right)\right)=x_{\sigma\left(\tau^{k}(\alpha)\right)}\left(c_{\tau^{k}(\alpha)} \lambda^{q^{k}}\right), \\
& F^{k}\left(\sigma\left(x_{\alpha}(\lambda)\right)\right)=F^{k}\left(x_{\sigma(\alpha)}\left(c_{\alpha} \lambda\right)\right)=x_{\tau^{k}(\sigma(\alpha))}\left(\left(c_{\alpha}\right)^{q^{k}} \lambda^{q^{k}}\right) .
\end{aligned}
$$

Or les actions de $\sigma$ et $F$ commutent, donc $\sigma\left(F^{k}\left(x_{\alpha}(\lambda)\right)\right)=F^{k}\left(\sigma\left(x_{\alpha}(\lambda)\right)\right)$ et $\sigma \circ \tau^{k}=\tau^{k} \circ \sigma$, ce qui entraîne le résultat.

Remarque 4.6. - Le lemme 4.5 entraîne que pour tout $\alpha \in \Phi$, si $\mathcal{O}$ est la $\tau$-orbite de $\alpha$, alors le coefficient $c_{\alpha}$ est dans $\mathbb{F}_{q^{|\mathcal{O}|}}$. 
LEMME 4.7. - On considère la $\tau$-orbite $\mathcal{O}=\left\{\alpha, \tau(\alpha), \ldots, \tau^{k-1}(\alpha)\right\}$ d'une racine $\alpha \in \Pi$. Soit $\mathcal{A}=\left\{\alpha, \sigma(\alpha), \ldots, \sigma^{n-1}(\alpha)\right\}$ la $\sigma$-orbite de $\alpha$. Soient $a \in\{1, \ldots, k\}$ le cardinal de la $\tau$-orbite de $\mathcal{A}$ et $o \in\{1, \ldots, n\}$ le cardinal de la $\sigma$-orbite de $\mathcal{O}$. Alors il existe un entier $r$ tel que $k=a \times r$ et $n=o \times r$.

Démonstration. - Pour tout entier $t$, on a

$$
\begin{aligned}
\sigma^{t}(\mathcal{O}) & =\left\{\sigma^{t}(\alpha), \tau\left(\sigma^{t}(\alpha)\right), \ldots, \tau^{k-1}\left(\sigma^{t}(\alpha)\right)\right\} \\
\tau^{t}(\mathcal{A}) & =\left\{\tau^{t}(\alpha), \sigma\left(\tau^{t}(\alpha)\right), \ldots, \sigma^{n-1}\left(\tau^{t}(\alpha)\right)\right\} .
\end{aligned}
$$

Par définition de $o$ et de $a$, on a $\sigma^{o}(\mathcal{O})=\mathcal{O}$ et $\tau^{a}(\mathcal{A})=\mathcal{A}$. De plus, on a l'égalité $\mathcal{O} \cup \cdots \cup \sigma^{o-1}(\mathcal{O})=\mathcal{A} \cup \cdots \cup \tau^{a-1}(\mathcal{A})$, ce qui entraîne que

1) $o \times k=a \times n$.

Par ailleurs, $\sigma^{n}(\mathcal{O})=\mathcal{O}$ et $\tau^{k}(\mathcal{A})=\mathcal{A}$; donc il existe deux entiers $u$ et $v$ tels que

2) $n=u \times o$ et

3) $k=v \times a$.

On obtient donc la relation $u=v$, ce qui conclut la preuve du lemme 4.7.

LEMME 4.8. - On suppose que $\sigma$ est quasi-central et que pour toute racine simple $\alpha \in \Pi$, on a $C_{\sigma, \alpha}=1$. Soit $\alpha \in \Pi$, soient $\mathcal{A}$ et $\mathcal{O}$ respectivement la $\sigma$-orbite et la $\tau$-orbite de $\alpha$, soit o le cardinal de la $\sigma$-orbite de $\mathcal{O}$ et a le cardinal de la $\tau$-orbite de $\mathcal{A}$, soit $i \in\{1, \ldots,|\mathcal{O}|\}$ tel que $\sigma^{o}(\alpha)=\tau^{i}(\alpha)$ et soit $j \in\{1, \ldots,|\mathcal{A}|\}$ tel que $\tau^{a}(\alpha)=\sigma^{j}(\alpha)$; enfin soit $r$ tel que $|\mathcal{A}|=r \times$ o. Alors on a

$$
\left(c_{\alpha} \cdots c_{\sigma^{o-1}(\alpha)}\right)^{1+q^{i}+\cdots+q^{(r-1) i}}=1 \quad \text { et } \quad\left(c_{\alpha} \cdots c_{\sigma^{j-1}(\alpha)}\right)^{1+q^{a}+\cdots+q^{(r-1) a}}=1 .
$$

Démonstration. - Il suffit d'écrire

$$
\begin{aligned}
\left(c_{\alpha}\right. & \left.\cdots c_{\sigma^{o-1}(\alpha)}\right)^{1+q^{i}+\cdots+q^{(r-1) i}} \\
& =c_{\alpha} \cdots c_{\sigma^{o-1}(\alpha)} c_{\alpha}^{q^{i}} \cdots c_{\sigma^{o-1}(\alpha)}^{q^{i}} \cdots c_{\alpha}^{q^{(r-1) i}} \cdots c_{\sigma^{o-1}(\alpha)}^{q^{(r-1) i}} \\
& =c_{\alpha} \cdots c_{\sigma^{o-1}(\alpha)} c_{\tau^{i}(\alpha)} \cdots c_{\sigma^{o-1}\left(\tau^{i}(\alpha)\right)} \cdots c_{\tau^{(r-1) i}(\alpha)} \cdots c_{\sigma^{o-1}\left(\tau^{(r-1) i}(\alpha)\right)} \\
& =c_{\alpha} \cdots c_{\sigma^{o-1}(\alpha)} c_{\sigma^{o}(\alpha)} \cdots c_{\sigma^{2 o-1}(\alpha)} \cdots c_{\sigma^{(r-1) o}(\alpha)} \cdots c_{\sigma^{r o-1}(\alpha)} \\
& =c_{\alpha} \cdots c_{\sigma^{|\mathcal{A}|-1}(\alpha)}=1
\end{aligned}
$$

On montre de la même façon que $\left(c_{\alpha} \cdots c_{\sigma^{j-1}(\alpha)}\right)^{1+q^{a}+\cdots+q^{(r-1) a}}=1$.

Lemme 4.9. - Soit $\alpha \in \Pi$; on note $H$ le sous-réseau de $\mathbb{Z}^{2}$ défini par

$$
H=\left\{(i, j) ; \tau^{i}(\alpha)=\sigma^{j}(\alpha)\right\} \text {. }
$$

Si $n$ est le cardinal de la $\sigma$-orbite de $\alpha$, et si a $>0$ est minimal tel qu'il existe un entier $\ell$ tel que $\tau^{a}(\alpha)=\sigma^{\ell}(\alpha)$ (si $\mathcal{A}$ est la $\sigma$-orbite de $\alpha$, alors a est le cardinal de la $\tau$-orbite de $\mathcal{A})$ et si $j$ est tel que $\tau^{a}(\alpha)=\sigma^{j}(\alpha)$, alors $H$ est engendré par les couples $(0, n)$ et $(a, j)$.

TOME $132-2004-\mathrm{N}^{\mathrm{O}} 2$ 
Démonstration. — Soit $p_{1}$ la première projection de $\mathbb{Z}^{2}$ sur $\mathbb{Z}$, alors l'image de $H$ par $p_{1}$ est un sous-groupe de $\mathbb{Z}$ égal à $a \cdot \mathbb{Z}$ par définition de $a$. Ainsi pour tout $(k, \ell) \in H$, il existe un entier $s$ tel que $k-s a=0$; on a alors $(0, \ell-s j) \in H$, c'est-à-dire $\sigma^{\ell-s j}(\alpha)=\alpha$, ce qui entraîne que $n$ divise $\ell-s j$. Donc, pour tout $(k, \ell) \in H$, il existe deux entiers $s$ et $t$ tels que $(k, \ell)=s(a, j)+t(0, n)$, ce qui montre que $H$ est engendré par les couples $(0, n)$ et $(a, j)$.

LEMME 4.10. - On suppose que $\sigma$ est quasi-central et que pour toute racine simple $\alpha \in \Pi$, on a $C_{\sigma, \alpha}=1$. Pour toute racine $\alpha \in \Pi$, si on note $\mathcal{O}$ la $\tau$ orbite de $\alpha, \mathcal{A}$ sa $\sigma$-orbite et a le cardinal de la $\tau$-orbite de $\mathcal{A}$ et $j$ l'élément de $\{1, \ldots, \mathcal{A}\}$ tel que $\tau^{a}(\alpha)=\sigma^{j}(\alpha)$, alors on peut choisir pour tout $\beta \in \mathcal{A}$, l'isomorphisme $x_{\beta}: \overline{\mathbb{F}}_{q}^{+} \rightarrow U_{\beta}$ de façon que $c_{\alpha} \cdots c_{\sigma^{j-1}(\alpha)}=1$.

Démonstration. - On s'est donné des isomorphismes $x_{\alpha}: \overline{\mathbb{F}}_{q}^{+} \rightarrow U_{\alpha}$ pour tout $\alpha \in \Phi$ tels que $F\left(x_{\alpha}(\lambda)\right)=x_{\tau(\alpha)}\left(\lambda^{q}\right)$ pour tout $\lambda \in \overline{\mathbb{F}}_{q}^{+}$. Soit $\left(a_{\alpha}\right)_{\alpha \in \Phi}$ une famille d'éléments de $\overline{\mathbb{F}}_{q}^{+}$; alors on peut définir la famille d'isomorphismes $\left(x_{\alpha}^{\prime}\right)_{\alpha \in \Phi}$ par la relation $x_{\alpha}^{\prime}(\lambda)=x_{\alpha}\left(a_{\alpha} \lambda\right)$ pour tout $\lambda \in \overline{\mathbb{F}}_{q}^{+}$.

Les isomorphismes vérifient $F\left(x_{\alpha}^{\prime}(\lambda)\right)=x_{\tau(\alpha)}^{\prime}\left(\lambda^{q}\right)$ pour tout $\alpha \in \Phi$, si et seulement si on a $a_{\tau(\alpha)}=a_{\alpha}^{q}$ : c'est l'hypothèse que l'on va faire sur les $a_{\alpha}$ désormais.

Pour tout $\alpha \in \Phi$, on définit $c_{\alpha}^{\prime}$ par $\sigma\left(x_{\alpha}^{\prime}(\lambda)\right)=x_{\sigma(\alpha)}^{\prime}\left(c_{\alpha}^{\prime} \lambda\right)$ pour tout $\lambda \in \overline{\mathbb{F}}_{q}^{+}$, alors $c_{\alpha}^{\prime}=c_{\alpha} \times a_{\alpha} / a_{\sigma(\alpha)}$. Pour tout $\alpha \in \Pi$, on a

$$
c_{\alpha}^{\prime} \cdots c_{\sigma|\mathcal{A}|-1}^{\prime}(\alpha)=C_{\sigma, \alpha}=1,
$$

où $\mathcal{A}$ est la $\sigma$-orbite de $\alpha$.

Soit $\alpha \in \Pi$, soit $a$ le cardinal de la $\tau$-orbite de $\mathcal{A}$ et $j$ l'élément $\operatorname{de}\{1, \ldots,|\mathcal{A}|\}$ tel que $\tau^{a}(\alpha)=\sigma^{j}(\alpha)$, alors $c_{\alpha}^{\prime} \cdots c_{\sigma^{j-1}(\alpha)}^{\prime}=a_{\alpha}^{1-q^{a}} c_{\alpha} \cdots c_{\sigma^{j-1}(\alpha)}$. On a vu (lemme 4.8) que

$$
\left(c_{\alpha} \cdots c_{\sigma^{j-1}(\alpha)}\right)^{1+q^{a}+\cdots+q^{(r-1) a}}=\left(c_{\alpha} \cdots c_{\sigma^{j-1}(\alpha)}\right)^{\left(q^{r a}-1\right) /\left(q^{a}-1\right)}=1 .
$$

Donc il existe $a_{\alpha} \in \mathbb{F}_{q|\mathcal{O}|}$, où $\mathcal{O}$ est la $\tau$-orbite de $\alpha$ tel que

$$
\left(a_{\alpha}\right)^{q^{a}-1}=c_{\alpha} \cdots c_{\sigma^{j-1}(\alpha)},
$$

ce qui termine la démonstration.

On suppose dans la suite que les isomorphismes $x_{\beta}$ vérifient la propriété du lemme 4.10 .

Notation 4.11. - Pour toute racine $\alpha \in \Pi$, si $\mathcal{O}$ est la $\tau$-orbite de $\alpha$, on définit l'isomorphisme $u_{\alpha}$ de $\mathbb{F}_{q|\mathcal{O}|}$ dans $U_{\mathcal{O}}^{F}$ par

$$
\lambda \longmapsto u_{\alpha}(\lambda)=x_{\alpha}(\lambda) \cdots x_{\tau^{|\mathcal{O}|-1}(\alpha)}\left(\lambda^{q^{|\mathcal{O}|-1}}\right) .
$$

BULletin DE LA SOCiÉtÉ MATHÉMATIQUE DE FRANCE 
Si $\tilde{\chi}$ est un caractère régulier normalisé de $U^{F} \cdot\langle\sigma\rangle$, il existe $\chi \in \operatorname{Irr}\left(U^{F}\right)$ régulier $\sigma$-stable tel que $\tilde{\chi}$ soit l'extension triviale de $\chi$ à $U^{F} \cdot\langle\sigma\rangle$. Pour tout $\alpha \in \Pi$, si $\mathcal{O}$ est la $\tau$-orbite de $\alpha$, on note $\chi_{\alpha}$ le morphisme de $\mathbb{F}_{q^{|\mathcal{O}|}}^{+}$dans $\mathbb{C}^{\times}$ défini par $\lambda \mapsto \chi \circ u_{\alpha}(\lambda)$. Alors le caractère $\chi$ est défini par la donnée de $\left(\chi_{\alpha}\right)_{\alpha \in \Pi}$ avec pour toute racine $\alpha \in \Pi$ les relations

$$
\chi_{\tau(\alpha)}\left(\lambda^{q}\right)=\chi_{\alpha}(\lambda)
$$

pour tout $\lambda \in \mathbb{F}_{q^{|\mathcal{O}|}}$, où $\mathcal{O}$ est la $\tau$-orbite de $\alpha$.

Soit $\left\{\alpha_{1}, \ldots \alpha_{r}\right\}$ un ensemble de représentants des $\tau$-orbites de $\Pi$. Alors, pour tout $u \in \prod_{i=1}^{r} u_{\alpha_{i}}\left(\lambda_{\alpha_{i}}\right)\left(U^{*}\right)^{F}$, on a la formule suivante qui ne dépend pas du choix de l'ensemble $\left\{\alpha_{1}, \ldots, \alpha_{r}\right\}$ :

$$
\chi(u)=\prod_{i=1}^{r} \chi_{\alpha_{i}}\left(\lambda_{\alpha_{i}}\right) .
$$

On note $\left\{\mathcal{O}_{1}, \ldots, \mathcal{O}_{s}\right\}$ un ensemble de représentants des $\sigma$-orbites de $\Pi / \tau$. Dans toute $\tau$-orbite $\mathcal{O}_{r}$, on choisit un élément $\alpha_{r}$. Pour tout $r \in\{1, \ldots, s\}$, $\mathcal{A}_{r}$ est la $\sigma$-orbite de $\alpha_{r}, a(r)$ le cardinal de la $\tau$-orbite de $\mathcal{A}_{r}$ et $o(r)$ le cardinal de la $\sigma$-orbite de $\mathcal{O}_{r}$. On fixe un entier $N$ multiple de $|\mathcal{O}|$ pour toute $\tau$-orbite $\mathcal{O}$ de racines de $\Pi$. On fixe un caractère $\phi_{0}$ de $\mathbb{F}_{q^{N}}^{+}$tel que la restriction de $\phi_{0}$ à $\mathbb{F}_{q}^{+}$soit non triviale.

LEMME 4.12. - On suppose que $\sigma$ est quasi-central et que $C_{\sigma, \alpha}=1$ pour toute racine simple $\alpha \in \Pi$. Pour tout $r \in\{1, \ldots, s\}$, pour tout caractère linéaire $\sigma$ stable $\chi_{r}$ du sous-groupe $\prod_{i=0}^{o(r)-1} U_{\sigma^{i}\left(\mathcal{O}_{r}\right)}^{F}$ de $\left(U / U^{*}\right)^{F}$, il existe un $d_{r} \in \mathbb{F}_{q^{a(r)}}$ tel que

$$
\begin{aligned}
& \chi_{r}\left(\prod_{i=0}^{o(r)-1} u_{\sigma^{i}\left(\alpha_{r}\right)}\left(\lambda_{\sigma^{i}\left(\alpha_{r}\right)}\right)\right) \\
& \quad=\phi_{0} \circ \operatorname{Tr}_{r}\left(d _ { r } \left(\lambda_{\alpha_{r}}+\left(1 / c_{\alpha_{r}}\right) \lambda_{\sigma\left(\alpha_{r}\right)}\right.\right. \\
& \left.\left.\quad+\cdots+\left(1 /\left(c_{\alpha_{r}} \cdots c_{\sigma^{o(r)-2}\left(\alpha_{r}\right)}\right)\right) \lambda_{\sigma^{o(r)-1}\left(\alpha_{r}\right)}\right)\right)
\end{aligned}
$$

où $\lambda_{\sigma^{i}\left(\alpha_{r}\right)} \in \mathbb{F}_{q^{\left|\mathcal{O}_{r}\right|}}$ et où $\operatorname{Tr}_{r}$ est la trace de $\mathbb{F}_{q^{\left|\mathcal{O}_{r}\right|}}$ dans $\mathbb{F}_{q^{a(r)}}$. Tout caractère linéaire $\sigma$-stable $\chi$ de $\left(U / U^{*}\right)^{F}$ est de la forme $\chi=\prod_{r=1}^{s} \chi_{r}$, où $\chi_{r}$ est comme ci-dessus. De plus, l'application

$$
\Psi:\left(\left(\left(U / U^{*}\right)^{F}\right)^{\wedge}\right)^{\sigma} \longrightarrow \prod_{r=1}^{s} \mathbb{F}_{q^{a(r)}}^{+}, \quad \chi \longmapsto\left(d_{1}, \ldots, d_{s}\right)
$$

est un isomorphisme et $\chi$ est régulier si et seulement si aucun des $d_{r}$ n'est nul.

TOME $132-2004-\mathrm{N}^{\mathrm{O}} 2$ 
Démonstration. - Soit $(s, t) \in H$ le sous-réseau du lemme 4.9. Alors, dans $\widetilde{U}=U / U^{*}$,

$$
\begin{aligned}
\sigma^{t}\left(u_{\alpha}(\lambda)\right) & =x_{\sigma^{t}(\alpha)}\left(c_{\alpha} \cdots c_{\sigma^{t-1}(\alpha)} \lambda\right) \cdots x_{\tau^{|\mathcal{O}|-1}\left(\sigma^{t}(\alpha)\right)}\left(\left(c_{\alpha} \cdots c_{\sigma^{t-1}(\alpha)} \lambda\right)^{q^{|\mathcal{O}|-1}}\right) \\
& =x_{\tau^{s}(\alpha)}\left(c_{\alpha} \cdots c_{\sigma^{t-1}(\alpha)} \lambda\right) \cdots x_{\tau^{|\mathcal{O}|+s-1}(\alpha)}\left(\left(c_{\alpha} \cdots c_{\sigma^{t-1}(\alpha)} \lambda\right)^{q^{|\mathcal{O}|-1}}\right) \\
& =u_{\tau^{s}(\alpha)}\left(c_{\alpha} \cdots c_{\sigma^{t-1}(\alpha)} \lambda\right) .
\end{aligned}
$$

Donc, si $\sigma^{t}(\alpha)=\tau^{s}(\alpha)$, alors $\sigma^{t}\left(u_{\alpha}(\lambda)\right)=u_{\tau^{s}(\alpha)}\left(c_{\alpha} \cdots c_{\sigma^{t-1}(\alpha)} \lambda\right)$, comme éléments de $U / U^{*}$.

Pour que $\chi$ soit un caractère régulier $\sigma$-stable de $U^{F}$, il faut et il suffit que pour tout $\alpha \in \Pi$, pour tout $\lambda \in \mathbb{F}_{q^{|\mathcal{O}|}}$, où $\mathcal{O}$ est la $\tau$-orbite de $\alpha$, on ait

$$
\chi_{\sigma(\alpha)}\left(c_{\alpha} \lambda\right)=\chi_{\alpha}(\lambda)
$$

c'est-à-dire, d'après ce qui précède, que pour tout couple $(s, t)$ tel que $\sigma^{t}(\alpha)=$ $\tau^{s}(\alpha)$, pour tout $\lambda \in \mathbb{F}_{q^{|\mathcal{O}|}}$, on ait

$$
\chi_{\sigma^{t}(\alpha)}\left(c_{\alpha} \cdots c_{\sigma^{t-1}(\alpha)} \lambda\right)=\chi_{\tau^{s}(\alpha)}\left(c_{\alpha} \cdots c_{\sigma^{t-1}(\alpha)} \lambda\right)=\chi_{\alpha}(\lambda) .
$$

Par le lemme 4.9, cette condition est équivalente à la condition suivante pour toute racine $\alpha \in \Pi$, où on note $\mathcal{O}$ la $\tau$-orbite de $\alpha, \mathcal{A}$ sa $\sigma$-orbite, a le cardinal de la $\tau$-orbite de $\mathcal{A}$, et où on définit $j$ comme l'élément de $\{1, \ldots,|\mathcal{O}|\}$ tel que $\sigma^{j}(\alpha)=\tau^{a}(\alpha)$

$$
\chi_{\tau^{a}(\alpha)}\left(c_{\alpha} \cdots c_{\sigma^{j-1}(\alpha)} \lambda\right)=\chi_{\alpha}(\lambda), \quad \text { pour tout } \lambda \in \mathbb{F}_{q^{|\mathcal{O}|}},
$$

c'est-à-dire $\chi_{\alpha}\left(\left(c_{\alpha} \cdots c_{\sigma^{j-1}(\alpha)} \lambda\right)^{q^{|\mathcal{O}|-a}}\right)=\chi_{\alpha}(\lambda)$, soit encore

$$
\chi_{\alpha}\left(\lambda^{q^{|\mathcal{O}|-a}}\right)=\chi_{\alpha}(\lambda) \text {, pour tout } \lambda \in \mathbb{F}_{q^{|\mathcal{O}|}},
$$

car on a fait en sorte que $c_{\alpha} \cdots c_{\sigma^{j-1}(\alpha)}=1$ grâce au lemme 4.10. Alors

$$
\chi_{\alpha}\left(\lambda^{q^{a}}\right)=\chi_{\alpha}\left(\left(\lambda^{q^{a}}\right)^{q^{|\mathcal{O}|-a}}\right)=\chi_{\alpha}\left(\lambda^{q^{|\mathcal{O}|}}\right)=\chi_{\alpha}(\lambda),
$$

pour tout $\lambda \in \mathbb{F}_{q|\mathcal{O}|}$.

Ainsi, si $\chi_{r}$ est un caractère linéaire $\sigma$-stable de $\prod_{i=0}^{o(r)-1} U_{\sigma^{i}\left(\mathcal{O}_{r}\right)}^{F}$ avec $r \in\{1, \ldots, s\}$, il existe un caractère $\phi_{r}$ de $\mathbb{F}_{q^{a(r)}}^{+}$tel que

$$
\begin{aligned}
& \chi_{r}\left(\prod_{i=0}^{o(r)-1} u_{\sigma^{i}\left(\alpha_{r}\right)}\left(\lambda_{\sigma^{i}\left(\alpha_{r}\right)}\right)\right) \\
& =\phi_{r} \circ \operatorname{Tr}_{r}\left(\lambda_{\alpha_{r}}+\left(1 / c_{\alpha_{r}}\right) \lambda_{\sigma\left(\alpha_{r}\right)} \cdots+\left(1 /\left(c_{\alpha_{r}} \cdots c_{\sigma^{o(r)-2}\left(\alpha_{r}\right)}\right)\right) \lambda_{\sigma^{o(r)-1}\left(\alpha_{r}\right)}\right),
\end{aligned}
$$

BULLETIN DE LA SOCIÉTÉ MATHÉMATIQUE DE FRANCE 
où $\lambda_{\sigma^{i}\left(\alpha_{r}\right)} \in \mathbb{F}_{q^{\left|\mathcal{O}_{r}\right|}}$ et où $\operatorname{Tr}_{r}$ est la trace de $\mathbb{F}_{q^{\left|\mathcal{O}_{r}\right|}}$ dans $\mathbb{F}_{q^{a(r)}}$. C'est-à-dire, il existe un $d_{r} \in \mathbb{F}_{q^{a(r)}}$ tel que

$$
\begin{aligned}
& \chi_{r}\left(\prod_{i=0}^{o(r)-1} u_{\sigma^{i}\left(\alpha_{r}\right)}\left(\lambda_{\sigma^{i}\left(\alpha_{r}\right)}\right)\right) \\
&=\phi_{0} \circ \operatorname{Tr}_{r}\left(d _ { r } \left(\lambda_{\alpha_{r}}+\left(1 / c_{\alpha_{r}}\right) \lambda_{\sigma\left(\alpha_{r}\right)}\right.\right. \\
&\left.\left.\quad+\cdots+\left(1 /\left(c_{\alpha_{r}} \cdots c_{\sigma^{o(r)-2}\left(\alpha_{r}\right)}\right)\right) \lambda_{\sigma^{o(r)-1}\left(\alpha_{r}\right)}\right)\right) .
\end{aligned}
$$

Et le caractère $\chi$ est $\sigma$-stable si et seulement si $\chi=\prod_{r=1}^{s} \chi_{r}$, où $\chi_{r}$ est comme ci-dessus, de plus, il est régulier si et seulement si les $d_{r}$ sont non nuls.

Les autres assertions du lemme 4.12 sont claires.

On peut appliquer le lemme 4.12, non seulement à $G \cdot\langle\sigma\rangle$ mais aussi au groupe réductif $\left(G^{\sigma}\right)^{0} \cdot\langle\sigma\rangle$. Cela montre que les caractères réguliers $\sigma$-stables de $U^{F} /\left(U^{*}\right)^{F}$ et les caractères réguliers de $\left(U^{\sigma}\right)^{F} /\left(\left(U^{\sigma}\right)^{*}\right)^{F}$ sont en bijection avec le même ensemble. La proposition 4.4 s'en déduit immédiatement.

Corollaire 4.13. - Supposons la condition $(\mathrm{RS})$ vérifiée. Alors les caractères réguliers $\sigma$-stables de $U^{F}$ sont paramétrés par $\mathcal{L}_{\left(T^{\sigma}\right)^{0}}^{-1}\left(\mathrm{Z}\left(\left(G^{\sigma}\right)^{0}\right)\right) / \mathrm{Z}\left(\left(G^{\sigma}\right)^{0}\right)$, où $\mathcal{L}_{\left(T^{\sigma}\right)^{0}}$ est l'application de Lang $t \mapsto t^{-1} F(t)$ de $\left(T^{\sigma}\right)^{0}$ dans $\left(T^{\sigma}\right)^{0}$.

Démonstration. — Selon la proposition 14.28 de [3] appliquée au groupe réductif connexe $\left(G^{\sigma}\right)^{0}, \mathcal{L}_{\left(T^{\sigma}\right)^{0}}^{-1}\left(\mathrm{Z}\left(\left(G^{\sigma}\right)^{0}\right)\right)$ agit transitivement sur l'ensemble des caractères réguliers de $\left(U^{\sigma}\right)^{F}$, et comme cette action a pour noyau $\mathrm{Z}\left(\left(G^{\sigma}\right)^{0}\right)$, les caractères réguliers de $\left(U^{\sigma}\right)^{F}$ sont paramétrés par l'ensemble

$$
\mathcal{L}_{\left(T^{\sigma}\right)^{0}}^{-1}\left(\mathrm{Z}\left(\left(G^{\sigma}\right)^{0}\right)\right) / \mathrm{Z}\left(\left(G^{\sigma}\right)^{0}\right) .
$$

La proposition précédente permet donc de conclure.

Précisons la bijection obtenue dans le corollaire 4.13 : on fixe un caractère régulier $\sigma$-stable $\chi_{1}$ de $U^{F}$ afin de bien définir ce paramétrage.

D'après le lemme 1.3 de $[2], \mathcal{L}_{\left(T^{\sigma}\right)^{0}}^{-1}\left(\mathrm{Z}\left(\left(G^{\sigma}\right)^{0}\right)\right) /\left(\mathrm{Z}\left(\left(G^{\sigma}\right)^{0}\right)\left(\left(T^{\sigma}\right)^{0}\right)^{F}\right)$ est isomorphe à $\mathrm{H}^{1}\left(F, \mathrm{Z}\left(\left(G^{\sigma}\right)^{0}\right)\right)$, donc les $\left(\left(T^{\sigma}\right)^{0}\right)^{F}$-orbites des caractères réguliers $\sigma$-stables de $U^{F}$ sont paramétrées par $\mathrm{H}^{1}\left(F, \mathrm{Z}\left(\left(G^{\sigma}\right)^{0}\right)\right)$. Comme $\left(T^{\sigma}\right)^{0}$ est connexe, l'application de Lang de $\left(T^{\sigma}\right)^{0} \rightarrow\left(T^{\sigma}\right)^{0}$ est surjective. De plus, on a $Z\left(\left(G^{\sigma}\right)^{0}\right) \subset\left(T^{\sigma}\right)^{0}$. Donc, pour tout $z \in \mathrm{H}^{1}\left(F, \mathrm{Z}\left(\left(G^{\sigma}\right)^{0}\right)\right)$, il existe $t \in\left(T^{\sigma}\right)^{0}$ tel que $t^{-1} F(t) \in z$ et $z$ paramètre la $\left(\left(T^{\sigma}\right)^{0}\right)^{F}$-orbite de ${ }^{t} \chi_{1}$.

\section{Définition des représentations de Gelfand-Graev}

5.1. Cas où $\boldsymbol{\sigma}$ est unipotent. - On suppose que $\sigma$ est rationnel quasicentral unipotent. On rappelle que, lorsque $\sigma$ est quasi-central unipotent, on a $\left(G^{\sigma}\right)^{0}=G^{\sigma}$ et $\left(T^{\sigma}\right)^{0}=T^{\sigma}$. Nous allons étendre toute représentation de

TOME $132-2004-\mathrm{N}^{\mathrm{O}} 2$ 
Gelfand-Graev $\sigma$-stable de $G^{F}$ en une représentation de Gelfand-Graev de $\widetilde{G}^{F}$. Selon la proposition 5.1 ci-dessous, il suffit d'induire de $U^{F} \cdot\langle\sigma\rangle$ à $\widetilde{G}^{F}$ les caractères réguliers normalisés de $U^{F} \cdot\langle\sigma\rangle$.

Proposition 5.1. - Si $\sigma$ est un automorphisme quasi-central unipotent rationnel de $G$, alors, tout caractère $\sigma$-stable de Gelfand-Graev de $G^{F}$ est l'induit d'un caractère régulier $\sigma$-stable de $U^{F}$.

Démonstration. - Les représentations de Gelfand-Graev de $G$ comme les $T^{F}$ orbites de caractères réguliers de $U^{F}$ sont paramétrées par $\mathrm{H}^{1}(F, \mathrm{Z}(G)$ ) (proposition 14.28 de [3]) de la façon suivante : on fixe un caractère régulier de $U^{F}$ que l'on note $\phi_{1}$. Soit $z \in \mathrm{H}^{1}(F, \mathrm{Z}(G))$, soit $t \in \mathcal{L}_{T}^{-1}(Z(G))$ tel que la classe de Fconjugaison de $\mathcal{L}(t)$ dans $\mathrm{Z}(G)$ soit $z$. On note $\dot{z}$ l'image dans $\mathcal{L}_{T}^{-1}(\mathrm{Z}(G)) / \mathrm{Z}(G)$ de $t$. Soit $\phi_{\dot{z}}$ le caractère régulier de $U^{F}$ défini par $\phi_{\dot{z}}={ }^{t} \phi_{1}$, alors on définit $\Gamma_{z}$ $\operatorname{par} \Gamma_{z}=\operatorname{Ind}_{U^{F}}^{G^{F}}\left(\phi_{\dot{z}}\right)$. On démontre que $\Gamma_{z}=\Gamma_{z^{\prime}}$ si et seulement si $z=z^{\prime}$.

Soit $z \in \mathrm{H}^{1}(F, \mathrm{Z}(G))$ tel que $\Gamma_{z}$ soit une représentation de Gelfand-Graev $\sigma$-stable de $G^{F}$, soit $t \in \mathcal{L}_{T}^{-1}(\mathrm{Z}(G))$ défini comme ci-dessus. Soit $\phi={ }^{t} \phi_{1}$, alors $\Gamma_{z}=\operatorname{Ind}_{U^{F}}^{G^{F}}(\phi)$ et ${ }^{\sigma} \Gamma_{z}=\operatorname{Ind}_{U^{F}}^{G^{F}}\left({ }^{\sigma} \phi\right)$. Par ailleurs, ${ }^{\sigma} \phi$ est un caractère régulier de $U^{F}$. Donc, comme $\Gamma_{z}$ est $\sigma$-stable, il existe $t^{\prime} \in \mathcal{L}_{T}^{-1}(Z(G))$ tel que la classe de F-conjugaison de $\mathcal{L}\left(t^{\prime}\right)$ dans $\mathrm{Z}(G)$ soit $z$, c'est-à-dire celle de $t$, et tel que ${ }^{\sigma} \phi={ }^{t^{\prime}} \phi_{1}$. Ainsi il existe $t^{\prime \prime} \in \mathrm{Z}(G)$ tel que $\mathcal{L}\left(t^{\prime}\right)=t^{\prime \prime-1} \mathcal{L}(t) F\left(t^{\prime \prime}\right)$, c'està-dire tel que $F\left(t^{\prime}\left(t t^{\prime \prime}\right)^{-1}\right)=t^{\prime}\left(t t^{\prime \prime}\right)^{-1}$. Donc il existe $t^{\prime \prime} \in \mathrm{Z}(G)$ et $t_{0} \in T^{F}$ tels que $t^{\prime}=t_{0} t t^{\prime \prime}$. Ainsi, on a

$$
{ }^{\sigma} \phi={ }^{\prime} \phi_{1}={ }^{t_{0}} t t^{\prime \prime} \phi_{1}={ }^{t_{0}} \phi
$$

avec $t_{0} \in T^{F}$. On a donc montré que la $T^{F}$-orbite de $\phi$ était $\sigma$-stable; il reste à prouver qu'elle contient un élément $\sigma$-stable.

Soit $k$ l'ordre de $\sigma$, alors ${ }^{\sigma^{k}} \phi=\phi$, c'est-à-dire $\sigma^{k-1}\left(t_{0}\right) \cdots \sigma\left(t_{0}\right) t_{0} \in \mathrm{Z}(G)$. Comme $\sigma$ est unipotent quasi-semi-simple, alors, d'après le (iii) du théorème 2.3, $T=\mathcal{L}_{\sigma}(T) \times T^{\sigma}$, où $\mathcal{L}_{\sigma}: t \rightarrow t^{-1} \sigma(t)$. Ainsi, on peut écrire $t_{0}=t^{-1} \sigma(t) t_{1}$ avec $t^{-1} \sigma(t) \in T^{F}$ et $t_{1} \in\left(T^{\sigma}\right)^{F}$. De plus, on peut choisir $t \in T^{F}$. En effet, $\mathcal{L}_{\sigma}\left(T^{F}\right)$ est inclus dans $\mathcal{L}_{\sigma}(T)^{F}$, or on a $\left|\mathcal{L}_{\sigma}\left(T^{F}\right)\right|=\left|T^{F}\right| /\left|\left(T^{F}\right)^{\sigma}\right|$ et $\left|\mathcal{L}_{\sigma}(T)^{F}\right|=\left|T^{F}\right| /\left|\left(T^{\sigma}\right)^{F}\right|$, d'où l'égalité $\mathcal{L}_{\sigma}\left(T^{F}\right)=\mathcal{L}_{\sigma}(T)^{F}$. On a alors

$$
\sigma^{k-1}\left(t_{0}\right) \cdots \sigma\left(t_{0}\right) t_{0} \in \mathrm{Z}(G) \Longleftrightarrow \sigma^{k}(t) t^{-1} t_{1}^{k} \in \mathrm{Z}(G)
$$

c'est-à-dire $t_{1}^{k} \in \mathrm{Z}(G)$ car $\sigma$ est d'ordre $k$. Ainsi pour toute racine $\alpha \in \Pi$, on a $\alpha\left(t_{1}^{k}\right)=\alpha\left(t_{1}\right)^{k}=1$, or $\alpha\left(t_{1}\right) \in \overline{\mathbb{F}}_{q}$ et $k$ est une puissance de la caractéristique de $\overline{\mathbb{F}}_{q}$; donc $\alpha\left(t_{1}\right)=1$ pour toute racine $\alpha \in \Pi$, c'est-à-dire $t_{1} \in \mathrm{Z}(G)$ et $t_{0} \in \mathcal{L}_{\sigma}(T) \times \mathrm{Z}(G)$. Soit $\phi^{\prime}={ }^{t^{-1}} \phi ;$ alors

$$
\sigma\left(\phi^{\prime}\right)={ }^{\sigma\left(t^{-1}\right) t_{0}} \phi={ }^{\sigma\left(t^{-1}\right) t^{-1} \sigma(t) t_{1}} \phi={ }^{t^{-1}} \phi=\phi^{\prime} .
$$

BULLETIN DE LA SOCIÉtÉ MATHÉMATIQUE DE FRANCE 
On a ainsi montré qu'il existait un caractère régulier $\phi^{\prime} \sigma$-stable de $U^{F}$ tel que $\Gamma=\operatorname{Ind}_{U^{F}}^{G^{F}}\left(\phi^{\prime}\right)$.

DÉfinition 5.2. - Pour tout $z \in \mathrm{H}^{1}\left(F, \mathrm{Z}\left(G^{\sigma}\right)\right)$, l'induit $\operatorname{Ind}_{U^{F} \cdot\langle\sigma\rangle}^{G^{F} \cdot\langle\sigma\rangle}\left({ }^{t} \widetilde{\chi}_{1}\right)$ (où on note ${ }^{t} \widetilde{\chi}_{1}$ l'extension normalisée de ${ }^{t} \chi_{1}$ à $\left.U^{F} \cdot\langle\sigma\rangle\right)$ ne dépend pas du choix de $t \in T^{\sigma}$ tel que $t^{-1} F(t) \in z$ et on définit la représentation de Gelfand-Graev $\Gamma_{z}^{\widetilde{G}}$ de $\widetilde{G}^{F}=G^{F} \cdot\langle\sigma\rangle$ par $\Gamma_{z}^{\widetilde{G}}=\operatorname{Ind}_{U^{F} \cdot\langle\sigma\rangle}^{G^{F} \cdot\langle\sigma\rangle}\left({ }^{t} \widetilde{\chi}_{1}\right)$. On définit la représentation de Gelfand-Graev $\Gamma_{z}^{G \cdot \sigma}$ de $G^{F} \cdot \sigma$ par $\Gamma_{z}^{G \cdot \sigma}=\operatorname{Res}_{G^{F} \cdot \sigma}^{\widetilde{G}^{F}}\left(\Gamma_{z}^{\widetilde{G}}\right)$.

5.2. Cas où $\sigma$ est semi-simple. - On suppose que $\sigma$ est rationnel quasicentral semi-simple. On suppose de plus que $\sigma$ vérifie l'hypothèse (RS).

On voudrait que toute représentation de Gelfand-Graev $\sigma$-stable de $G^{F}$ soit étendue en au moins une représentation de Gelfand-Graev de $\widetilde{G}^{F}$. Selon la proposition 5.1, sous l'hypothèse $\sigma$ unipotent quasi-central, toute représentation de Gelfand-Graev $\sigma$-stable de $G^{F}$ est l'induite d'un caractère régulier $\sigma$-stable de $U^{F}$. La proposition 5.1 n'est plus vraie lorsque l'on supprime l'hypothèse $\sigma$ unipotent. On ne peut plus se contenter comme lorsque $\sigma$ est unipotent quasicentral de définir les représentations de Gelfand-Graev de $\widetilde{G}^{F}$ comme les induites de $U^{F} \cdot\langle\sigma\rangle$ à $\widetilde{G}^{F}$ des extensions à $U^{F} \cdot\langle\sigma\rangle$ des caractères réguliers $\sigma$-stables de $U^{F}$.

Par ailleurs, lorsque $\sigma$ est semi-simple, $G^{\sigma}$ et $T^{\sigma}$ ne sont pas toujours connexes; donc pour $z \in \mathrm{H}^{1}\left(F, \mathrm{Z}\left(G^{\sigma}\right)\right)$, il n'existe plus forcément $t \in T^{\sigma}$ tel que $t^{-1} F(t) \in z$. Cependant $T$ est connexe, donc l'application de Lang $\mathcal{L}_{T}: T \rightarrow T$ est surjective et comme $\mathrm{Z}\left(G^{\sigma}\right) \subset T$, pour tout $z \in \mathrm{H}^{1}\left(F, \mathrm{Z}\left(G^{\sigma}\right)\right)$, il existe $t \in T$ tel que $t^{-1} F(t) \in z$. Alors ${ }^{t} \sigma$ est un élément rationnel quasi-central de $\widetilde{G}$; on a $\widetilde{G}^{F}=G^{F} \cdot\left\langle{ }^{t} \sigma\right\rangle$ et $G^{F} \cdot \sigma=G^{F} \cdot{ }^{t} \sigma$ et le caractère régulier ${ }^{t} \chi_{1}$ est ${ }^{t} \sigma$-stable. De plus, $\Gamma=\operatorname{Ind}_{U^{F}}^{G^{F}} \chi_{1}$ est une représentation de Gelfand-Graev $\sigma$-stable de $G^{F}$.

DÉFINITION 5.3. - Soit $z \in \mathrm{H}^{1}\left(F, \mathrm{Z}\left(G^{\sigma}\right)\right.$ ). Alors $\operatorname{Ind}_{U^{F} \cdot{ }^{t}{ }_{\sigma}}^{G^{F}}\left({ }^{t} \widetilde{\chi}_{1}\right)$ (où ${ }^{t} \widetilde{\chi}_{1}$ est l'extension normalisée de ${ }^{t} \chi_{1}$ à $\left.U^{F} \cdot\left\langle{ }^{t} \sigma\right\rangle\right)$ ne dépend pas du choix de $t \in T$ tel que $t^{-1} F(t) \in z$ et on définit la représentation de Gelfand-Graev $\Gamma_{z}^{\widetilde{G}}$ de $\widetilde{G}^{F}=$ $G^{F} \cdot\langle\sigma\rangle$ par $\Gamma_{z}^{\widetilde{G}}=\operatorname{Ind}_{U^{F} \cdot\left\langle{ }^{t} \sigma\right\rangle}^{\widetilde{G}^{F}}\left({ }^{t} \widetilde{\chi}_{1}\right)$. On définit la représentation de GelfandGraev $\Gamma_{z}^{G \cdot \sigma} \operatorname{de} G^{F} \cdot \sigma \operatorname{par} \Gamma_{z}^{G \cdot \sigma}=\operatorname{Res}_{G^{F} \cdot \sigma}^{\widetilde{G}^{F}}\left(\Gamma_{z}^{\widetilde{G}}\right)$.

\section{Propriétés des représentations de Gelfand-Graev}

On suppose que $\sigma$ est rationnel quasi-central unipotent ou semi-simple.

Proposition 6.1. - (i) La restriction à $G^{F}$ d'une représentation de GelfandGraev de $\widetilde{G}^{F}$ est une représentation de Gelfand-Graev de $G^{F}$.

TOME $132-2004-\mathrm{N}^{\mathrm{O}} 2$ 
(ii) Les composantes irréductibles des représentations de Gelfand-Graev de $\widetilde{G}^{F}=G^{F} \cdot\langle\sigma\rangle$ sont toutes de multiplicité 1 .

Démonstration. - Soit $z \in \mathrm{Z}\left(G^{\sigma}\right)$, alors $\operatorname{Res}_{G^{F}}^{\widetilde{G}^{F}}\left(\Gamma_{z}^{\widetilde{G}}\right)$ est un caractère de Gelfand-Graev de $G^{F}$ car si $t \in T$ est tel que $\mathcal{L}(t) \in z$ (on choisit $t \in T^{\sigma}$ si $\sigma$ est unipotent), alors $\operatorname{Res}_{G^{F}} \widetilde{G}^{F}\left(\Gamma_{z}^{\widetilde{G}}\right)=\operatorname{Ind}_{U^{F}}^{G^{F}}\left({ }^{t} \chi_{1}\right)$.

Or ${ }^{t} \chi_{1}$ est un caractère régulier de $U^{F}$ et on retrouve bien un caractère de Gelfand-Graev de $G^{F}$. On peut décomposer $\Gamma_{z}^{\widetilde{G}}$ sous la forme $\Gamma_{z}^{\widetilde{G}}=\sum_{\chi \in \operatorname{Irr}\left(\widetilde{G}^{F}\right)} c_{\chi} \chi$, alors $\Gamma_{z \mid G^{F}}^{\widetilde{G}}=\sum_{\chi \in \operatorname{Irr}\left(\widetilde{G}^{F}\right)} c_{\chi} \chi_{\mid G^{F}}$. Par le théorème de Clifford, pour tout $\chi \in \operatorname{Irr}\left(\widetilde{G}^{F}\right)$, on a $\chi_{\mid G^{F}}=e \sum_{i=1}^{t} \gamma_{i}$, où $\gamma_{1}, \ldots, \gamma_{t}$ sont les conjugués d'un même caractère irréductible $\gamma$ de $G^{F}$ dans $\widetilde{G}^{F}$. Or le fait que $\Gamma_{z}^{\widetilde{G}} \mid G^{F}$ soit une représentation de Gelfand-Graev de $G^{F}$ entraîne que ses composantes irréductibles sont de multiplicité 1 ( $c f$. [3, th. 14.30]) et donc que pour tout $\chi \in \operatorname{Irr}\left(\widetilde{G}^{F}\right), c_{\chi}<2$. Donc les composantes irréductibles de $\Gamma_{z}^{\widetilde{G}}$ sont de multiplicité 1.

Soit $L$ un sous-groupe de Levi rationnel et $\sigma$-stable contenant $T$ d'un sousgroupe parabolique rationnel $\sigma$-stable $P$ de $G$ contenant $B$.

LEMME 6.2. - L'inclusion $\mathrm{Z}\left(G^{\sigma}\right) \subset \mathrm{Z}\left(L^{\sigma}\right)$ induit une application surjective $h_{L^{\sigma}}: \mathrm{H}^{1}\left(F, \mathrm{Z}\left(G^{\sigma}\right)\right) \rightarrow \mathrm{H}^{1}\left(F, \mathrm{Z}\left(L^{\sigma}\right)\right)$

Démonstration. - Lorsque $\sigma$ est unipotent, $G^{\sigma}$ est un groupe réductif connexe et $L^{\sigma}$ un sous-groupe de Levi de $G^{\sigma}$, le résultat provient alors du lemme 14.31 de [3]. Supposons maintenant que $\sigma$ est semi-simple. Soit $I$ le sous-ensemble de $\Pi$ tel que $L$ soit le sous-groupe de Levi standard $L_{I}$. Alors $I$ est $\sigma$-stable et

$$
\mathrm{Z}\left(G^{\sigma}\right)=\bigcap_{\alpha \in \Pi} \operatorname{Ker} \alpha_{\mid T^{\sigma}} \subset \mathrm{Z}\left(L^{\sigma}\right)=\bigcap_{\alpha \in I} \operatorname{Ker} \alpha_{\mid T^{\sigma}} .
$$

En fait, on obtient la suite exacte suivante :

$$
\mathrm{H}^{1}\left(\mathrm{Z}\left(G^{\sigma}\right)\right) \longrightarrow \mathrm{H}^{1}\left(\mathrm{Z}\left(L^{\sigma}\right)\right) \longrightarrow \mathrm{H}^{1}\left(\mathrm{Z}\left(L^{\sigma}\right) / \mathrm{Z}\left(G^{\sigma}\right)\right) .
$$

Donc il suffit de montrer que $\mathrm{Z}\left(L^{\sigma}\right) / \mathrm{Z}\left(G^{\sigma}\right)$ est connexe.

Le groupe $\mathrm{Z}\left(L^{\sigma}\right) / \mathrm{Z}\left(G^{\sigma}\right)$ est le centre de $L^{\sigma} / \mathrm{Z}\left(G^{\sigma}\right)$; on va montrer que $L^{\sigma} / \mathrm{Z}\left(G^{\sigma}\right)$ est un sous-groupe de Levi du groupe réductif connexe de centre trivial $G^{\sigma} / \mathrm{Z}\left(G^{\sigma}\right)$. Cela nous permettra de conclure car, par le (ii) du lemme 13.14 de [3], si $H$ est un groupe réductif connexe de centre connexe, alors le centre de tout sous-groupe de Lévi de $H$ est encore connexe.

Comme $\sigma$ est quasi-central, et comme $G$ est un groupe réductif connexe, on a $G^{\sigma}=\left(G^{\sigma}\right)^{0} \times \mathrm{Z}\left(G^{\sigma}\right)$ (théorème 2.3 , (iii)), donc $G^{\sigma} / \mathrm{Z}\left(G^{\sigma}\right)$ est un groupe réductif connexe de centre trivial. Par ailleurs, si on pose $U_{I}=\left\langle U_{\alpha} ; \alpha \in I\right\rangle$ et $U_{I}^{-}=\left\langle U_{\alpha} ;(-\alpha) \in I\right\rangle$, alors, par les résultats généraux de la section 2 , on a $L^{\sigma}=\left\langle T^{\sigma},\left(U_{I}\right)^{\sigma},\left(U_{I}^{-}\right)^{\sigma}\right\rangle$ et $\left(L^{\sigma}\right)^{0}=\left\langle\left(T^{\sigma}\right)^{0},\left(U_{I}\right)^{\sigma},\left(U_{I}^{-}\right)^{\sigma}\right\rangle$ et comme 
$T^{\sigma}=\left(T^{\sigma}\right)^{0} \times \mathrm{Z}\left(G^{\sigma}\right)$, le groupe $L^{\sigma} / \mathrm{Z}\left(G^{\sigma}\right)$ est connexe et c'est un sous-groupe de Levi de $G^{\sigma} / \mathrm{Z}\left(G^{\sigma}\right)$.

On définit les représentations de Gelfand-Graev de $L^{F} \cdot \sigma$ comme celles de $G^{F} \cdot \sigma$ dans la section 5 . On pose

$$
\phi_{1}=\operatorname{Res}_{U^{F} \cap L^{F}}^{U^{F}}\left(\chi_{1}\right)
$$

et on définit pour tout $t \in \mathcal{L}_{T}^{-1}\left(\mathrm{Z}\left(G^{\sigma}\right)\right)$, le caractère ${ }^{t} \tilde{\phi}_{1}$ de $\operatorname{Irr}\left(\left(L^{F} \cap U^{F}\right) \cdot\left\langle{ }^{t} \sigma\right\rangle\right)$ comme l'extension normalisée de ${ }^{t} \phi_{1}$ à $\left(U^{F} \cap L^{F}\right) \cdot\left\langle{ }^{t} \sigma\right\rangle$.

Remarque 6.3. - On a $\left.\left({ }^{t} \widetilde{\chi}_{1}\right)\right|_{\left(L^{F} \cap U^{F}\right) \cdot{ }_{\sigma}}=\left({ }^{t} \tilde{\phi}_{1}\right)$.

Proposition 6.4. - Pour tout $z \in \mathrm{H}^{1}\left(F, \mathrm{Z}\left(G^{\sigma}\right)\right)$, on a

$$
{ }^{*} \mathrm{R}_{L \cdot \sigma}^{G \cdot \sigma}\left(\Gamma_{z}^{G \cdot \sigma}\right)=\Gamma_{h_{L} \sigma(z)}^{L \cdot \sigma},
$$

où ${ }^{*} \mathrm{R}_{L \cdot \sigma}^{G \cdot \sigma}$ désigne la restriction de Harish-Chandra généralisée aux groupes non connexes comme dans [4].

Démonstration. - La propriété de l'énoncé est équivalente à : pour toute fonction de $\sigma$-classe $f$ de $L^{F} \cdot \sigma$, on a

$$
\left\langle R_{L \cdot \sigma}^{G \cdot \sigma}(f), \Gamma_{z}^{G \cdot \sigma}\right\rangle_{G^{F} \cdot \sigma}=\left\langle f, \Gamma_{h_{L} \sigma(z)}^{L \cdot \sigma}\right\rangle_{L^{F} \cdot \sigma}
$$

Soit $t \in T$ tel que $\mathcal{L}_{T}(t) \in z$ (si c'est possible, ce qui est toujours le cas lorsque $\sigma$ est unipotent, on choisit $t$ dans $T^{\sigma}$; on a alors ${ }^{t} \sigma=\sigma$ ). Alors

$$
\begin{aligned}
\left\langle f, \Gamma_{h_{L} \sigma(z)}^{L \cdot \sigma}\right\rangle_{L^{F} \cdot \sigma} & =\left\langle f, \operatorname{Ind}_{\left(L^{F} \cap U^{F}\right) \cdot{ }_{\sigma} \sigma}^{L^{F} \sigma}\left({ }^{t} \tilde{\phi}_{1}\right)\right\rangle_{L^{F} \cdot \sigma} \\
& =\left\langle\operatorname{Res}_{\left(L^{F} \cap{ }^{F} U^{F}\right) \cdot{ }^{F} \sigma}^{L^{F}}(f),\left({ }^{t} \tilde{\phi}_{1}\right)\right\rangle_{\left(L^{F} \cap U^{F}\right) \cdot{ }^{t} \sigma}
\end{aligned}
$$

Donc la propriété de l'énoncé est équivalente à : pour toute fonction de $\sigma$-classe de $L^{F} \cdot \sigma$, on a

$$
\left\langle R_{L \cdot \sigma}^{G \cdot \sigma}(f), \Gamma_{z}^{G \cdot \sigma}\right\rangle_{G^{F} \cdot \sigma}=\left\langle f,\left({ }^{t} \tilde{\phi}_{1}\right)\right\rangle_{\left(L^{F} \cap U^{F}\right) \cdot{ }_{\sigma}} \cdot
$$

On définit la fonction $\tilde{f}$ sur $P^{F} \cdot \sigma$ par $f \times$ Id dans la décomposition $P^{F} \cdot \sigma=$ $\left(L^{F} \cdot \sigma\right) V^{F}$, où $V$ est le radical unipotent de $P$. Alors on a aussi $\tilde{f}=f \times$ Id dans la décomposition $P^{F \cdot t} \sigma=\left(L^{F} \cdot{ }^{t} \sigma\right) V^{F}$.

LeMme 6.5. - On a $R_{L \cdot \sigma}^{G \cdot \sigma}(f)=\operatorname{Ind}_{P}^{G^{F} \cdot t_{\sigma} t_{\sigma}}(\tilde{f})$.

TOME $132-2004-\mathrm{N}^{\mathrm{O}} 2$ 
Démonstration. - Soit $g \in G^{F}$, alors

$$
\begin{aligned}
& \operatorname{Ind}_{P}^{G^{F} \cdot t_{\sigma}}{ }^{t}(\tilde{f})(g \cdot \sigma) \\
& =1 /\left|P^{F}\right| \sum_{\left\{x \in G^{F} \mid\left(x t \sigma\left(t^{-1}\right) \cdot \sigma\right) g \cdot \sigma\left(x t \sigma\left(t^{-1}\right) \cdot \sigma\right)^{-1} \in P^{F} . t_{\sigma}\right\}} \tilde{f}\left(\left(x t \sigma\left(t^{-1}\right) \cdot \sigma\right) g \cdot \sigma(x)^{-1}\right) \\
& =1 /\left|P^{F}\right| \sum_{\left\{y \in G^{F} \mid y \cdot \sigma g \cdot \sigma(y \cdot \sigma)^{-1} \in P^{F} \cdot \sigma\right\}} \tilde{f}\left(y \cdot \sigma g \cdot \sigma(y \cdot \sigma)^{-1}\right) \\
& \left(\operatorname{car} t^{-1} \sigma(t) \in T^{F} \text { et } P^{F} \cdot{ }^{t} \sigma=P^{F} \cdot \sigma\right) \\
& =\operatorname{Ind}_{P F \cdot \sigma}^{G^{F} \cdot \sigma}(\tilde{f})(g \cdot \sigma) \quad \text { (induction de Harish-Chandra) } \\
& =R_{L \cdot \sigma}^{G \cdot \sigma}(f)(g \cdot \sigma) .
\end{aligned}
$$

Ce qui termine la preuve du lemme 6.5.

Ainsi, le membre de gauche de l'égalité (1) est égal à

$$
\left\langle\operatorname{Ind}_{P F \cdot t_{\sigma}}^{G^{F} \cdot{ }_{\sigma}^{t}}(\tilde{f}), \operatorname{Ind}_{U^{F} \cdot t_{\sigma}}^{G^{F}}{ }^{t_{\sigma}}\left({ }^{t} \widetilde{\chi}_{1}\right)\right\rangle_{G^{F} \cdot t_{\sigma}},
$$

c'est-à-dire par la formule de Mackey pour les fonctions de ${ }^{t} \sigma$-classes, à

$$
\begin{array}{r}
\sum_{g \in\left(P^{F} \backslash G^{F} / U^{F}\right)^{t_{\sigma}}}\left\langle\tilde{f}, \operatorname{Ind}_{\left(P^{F} \cap P^{g}\left(U^{F}\right)\right) \cdot t_{\sigma}}^{P^{F} \cdot t_{\sigma}} \circ \operatorname{Res}_{\left.\left(P^{F} \cap^{g}\left(U^{F}\right) \cdot t_{\sigma}{ }^{F}\right)\right) \cdot{ }_{\sigma}}{ }^{g}\left({ }^{t} \widetilde{\chi}_{1}\right)\right\rangle_{P^{F} \cdot t_{\sigma}} \\
=\sum_{g \in\left(P^{F} \backslash G^{F} / U^{F}\right)^{t_{\sigma}}}\left\langle\tilde{f},{ }^{g}\left({ }^{t} \widetilde{\chi}_{1}\right)\right\rangle_{P^{F} \cdot t_{\sigma} \cap{ }^{g}\left(U^{F}\right) \cdot t_{\sigma}} .
\end{array}
$$

Si $L$ est le sous-groupe de Levi standard $L_{I}$, alors $P \backslash G / U$ est en bijection avec l'ensemble des éléments $I$-réduits de $W=\mathrm{N}_{G}(T) / T$ (voir [3, lemme 5.5]). Donc l'ensemble $\left(P^{F} \backslash G^{F} / U^{F}\right)^{t} \sigma$ est en bijection avec l'ensemble des éléments $I$-réduits ${ }^{t} \sigma$-stables de $W^{F}$; par ailleurs, comme ${ }^{t} \sigma$ est quasi-central, tout élément ${ }^{t} \sigma$-stable de $W^{F}=\mathrm{N}_{G}(T)^{F} / T^{F}$ a un représentant ${ }^{t} \sigma$-stable dans $\mathrm{N}_{G}(T)^{F}$. Ainsi $\left(P^{F} \backslash G^{F} / U^{F}\right)^{t} \sigma$ est en bijection avec un ensemble de représentants ${ }^{t} \sigma$ stables dans $\mathrm{N}_{G}(T)^{F}$ des éléments $I$-réduits ${ }^{t} \sigma$-stables de $W^{F}$. Soit $n$ un tel élément qui relève $w \in W^{F}$; alors on a

$$
P^{F} \cdot{ }^{t} \sigma \cap{ }^{n} U^{F} \cdot{ }^{t} \sigma=\left(\left(L^{F} \cap{ }^{n} U^{F}\right) \cdot{ }^{t} \sigma\right) \cdot\left(V^{F} \cap{ }^{n} U^{F}\right)
$$

et la restriction de $\tilde{f}$ à $\left(P^{F} \cap{ }^{n} U^{F}\right) \cdot{ }^{t} \sigma$ est égale à $\operatorname{Res}_{\left(L^{F} \cap^{n} U^{F}\right) \cdot t_{\sigma}}^{L^{F}}(f) \times \operatorname{Id}$ dans cette décomposition. On a donc

$$
\begin{aligned}
& \left\langle\tilde{f},{ }^{n}\left({ }^{t} \widetilde{\chi}_{1}\right)\right\rangle_{\left(P^{F} \cap^{n} U^{F}\right) \cdot t_{\sigma}} \\
& \quad=\left\langle\operatorname{Res}_{\left(L^{F} \cap^{F} \cdot{ }^{n} U^{F}\right) \cdot{ }_{\sigma}}(f),{ }^{n}\left({ }^{t} \widetilde{\chi}_{1}\right)\right\rangle_{L^{F} \cdot t_{\sigma} \cap^{n} U^{F} \cdot t_{\sigma}}\left\langle\mathrm{Id},{ }^{t} \chi_{1}\right\rangle_{n^{-1} V^{F} \cap U^{F}} .
\end{aligned}
$$

Comme ${ }^{t} \chi_{1}$ est un caractère régulier ${ }^{t} \sigma$-stable de $U^{F},\left\langle\operatorname{Id},{ }^{t} \chi_{1}\right\rangle_{n^{-1} V^{F} \cap U^{F}}$

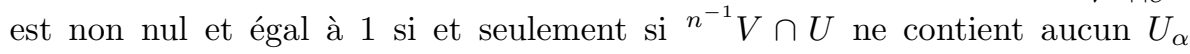
pour $\alpha \in \Pi$.

BULletin DE LA SOCiÉtÉ MATHÉmATiQUe DE FRANCE 
LEMME 6.6. - ${ }^{-1} V \cap U$ ne contient aucun $U_{\alpha}$ tel que $\alpha$ soit une racine simple si et seulement si $w=w_{0}^{I} w_{0}$ où $w_{0}^{I}$ est l'élément de plus grande longueur de $W_{I}$ et $w_{0}$ l'élément de plus grande longueur de $W$.

Démonstration. — On a

$$
U=\prod_{\alpha \in \Phi^{+}} U_{\alpha} \quad \text { et } \quad V=\prod_{\left\{\alpha \in \Phi^{+}-\langle I\rangle^{+}\right\}} U_{\alpha}=\prod_{\left\{\alpha \in \Phi^{+} \cap^{w_{0}^{I}} \Phi^{+}\right\}} U_{\alpha},
$$

où $w_{0}^{I}$ est le plus grand élément de $W_{I}$. Donc

$$
{ }^{n^{-1}} V \cap U=\prod_{\left\{\alpha \in \Phi^{+} \cap w^{-1} \Phi^{+} \cap^{w^{-1}} w_{0}^{I} \Phi^{+}\right\}} U_{\alpha}
$$

Or $w$ est $I$-réduit, donc, $\ell\left(w\left(w_{0}^{I}\right)^{-1}\right)=\ell(w)+\ell\left(\left(w_{0}^{I}\right)^{-1}\right)$ et

$$
\Phi^{+} \cap w^{-1} \Phi^{+} \cap w^{-1} w_{0}^{I} \Phi^{+}=\Phi^{+} \cap w^{-1} w_{0}^{I} \Phi^{+} .
$$

Ce qui entraîne que $n^{n^{-1}} V \cap U={ }^{-1} n_{0}^{I} V \cap U$, où $n_{0}^{I}$ est un représentant $\sigma$-stable de $w_{0}^{I}$ dans $\mathrm{N}_{G}(T)^{F}$ et ce groupe ne contient aucun $U_{\alpha}$ où $\alpha \in \Pi$ si et seulement si $\left(w_{0}^{I}\right)^{-1} w$ envoie toute racine simple positive sur une racine négative, c'està-dire si et seulement si $\left(w_{0}^{I}\right)^{-1} w=w_{0}$, où $w_{0}$ est l'élément de plus grande longueur de $W$. D'où le lemme 6.6.

En tenant compte du lemme ci-dessus, du fait que $w_{0}$ envoie les racines positives sur l'ensemble des racines négatives, du fait que $w_{0}^{I}$ change le signe des racines qui sont dans le système de racines de $L$ et seulement le leur et du fait que $w_{0}$ et $w_{0}^{I}$ sont ${ }^{t} \sigma$-stables (car $W$ et $W_{I}$ le sont), on a $L \cap{ }^{n} U=L \cap U$. Ainsi

$$
\left\langle R_{L \cdot \sigma}^{G \cdot \sigma}(f), \Gamma_{z}^{G \cdot \sigma}\right\rangle_{G^{F} \cdot \sigma}=\left\langle\operatorname{Res}_{\left(L^{F} \cap U^{F}\right) \cdot{ }_{\sigma}}^{L^{F}}(f),{ }^{n}\left({ }^{t} \widetilde{\chi}_{1}\right)\right\rangle_{\left(L^{F} \cap U^{F}\right) \cdot{ }^{t} \sigma} \cdot
$$

Par la remarque 6.3 , on obtient alors

$$
\left\langle R_{L \cdot \sigma}^{G \cdot \sigma}(f), \Gamma_{z}^{G \cdot \sigma}\right\rangle_{G^{F} \cdot \sigma}=\left\langle f,{ }^{n}\left({ }^{t} \tilde{\phi}_{1}\right)\right\rangle_{\left(L^{F} \cap U^{F}\right) \cdot{ }^{t} \sigma} .
$$

De plus, si on pose $n=t^{\prime} w$ avec $t^{\prime} \in T^{F}$, alors $n$ agit par conjugaison sur $L^{F} \cap U^{F}$ de la même façon que $t^{\prime}$ et les caractères ${ }^{n}\left({ }^{t} \tilde{\phi}_{1}\right)$ et $\left({ }^{t} \tilde{\phi}_{1}\right)$ sont dans la même $\left(T^{\sigma}\right)^{F}$-orbite : on en déduit la proposition 6.4 .

\section{Relation avec les éléments réguliers}

On considère la définition suivante de la dualité introduite par [4] pour les groupes réductifs non connexes :

DÉFinition 7.1. - Soit $(T, B)$ un couple formé d'un tore maximal de $G$ et d'un sous-groupe de Borel le contenant, tous deux rationnels et $\sigma$-stables. Alors le Frobenius agit sur le système de racines de $G$ par rapport à $T$; on note $\tau$

TOME $132-2004-\mathrm{N}^{\mathrm{O}} 2$ 
cette action. Alors $\tau$ préserve la base $\Pi$ du système de racines de $G$ par rapport à $T \subset B$; de plus, l'opérateur de dualité est défini par

$$
\mathrm{D}_{G \cdot \sigma}=\sum_{I \in \mathcal{P}(\Pi / \tau)^{\sigma}}(-1)^{|I / \sigma|} \mathrm{R}_{L_{I} \cdot \sigma}^{G \cdot \sigma} \circ{ }^{*} \mathrm{R}_{L_{I} \cdot \sigma}^{G \cdot \sigma},
$$

où $\Pi / \tau$ est l'ensemble des orbites de $\tau$ dans la base $\Pi$ et où $\mathcal{P}(\Pi / \tau)$ est l'ensemble des parties de $\Pi / \tau$.

D'après $[4$, cor. 3.12], la dualité est une involution isométrique.

Proposition 7.2. - (i) Le dual de toute représentation de Gelfand-Graev de $G^{F} \cdot \sigma$ est nul en dehors des éléments unipotents (resp. pseudo-unipotents) réguliers lorsque $\sigma$ est unipotent (resp. semi-simple).

(ii) Dans les deux cas, on a

$$
\left\langle\Gamma_{z}^{G \cdot \sigma},(-1)^{|\Pi / \tau \times \sigma|} \mathrm{D}_{G \cdot \sigma} \Gamma_{z^{\prime}}^{G \cdot \sigma}\right\rangle_{G^{F} \cdot \sigma}=\left|\mathrm{Z}\left(\left(G^{\sigma}\right)^{F}\right)\right| \delta_{z, z^{\prime}} .
$$

Démonstration. - (i) En utilisant la proposition 6.4, on obtient

$$
\mathrm{D}_{G \cdot \sigma} \Gamma_{z}^{G \cdot \sigma}=\sum_{I \in \mathcal{P}(\Pi / \tau)^{\sigma}}(-1)^{|I / \sigma|} \mathrm{R}_{L_{I} \cdot \sigma}^{G \cdot \sigma}\left(\Gamma_{h_{\left(L_{I}\right)^{\sigma}(z)}}^{L_{I} \cdot \sigma}\right) .
$$

Comme $\mathrm{R}_{L_{I} \cdot \sigma}^{G \cdot \sigma}$ est une induction de Harish-Chandra, si $P_{I}$ est le sous-groupe parabolique rationnel $\sigma$-stable de $G$ contenant $L_{I}$ et de radical unipotent $V_{I}$, en utilisant le lemme 6.5 et la remarque 6.3 , on obtient

$\left.\mathrm{R}_{L_{I} \cdot \sigma}^{G \cdot \sigma}\left(\Gamma_{h_{\left(L_{I}\right)} \sigma(z)}^{L_{I} \cdot \sigma}\right)=\operatorname{Ind}_{\left(P_{I}\right)^{F} \cdot t_{\sigma}}^{G^{F} \cdot t_{\sigma}}\left[\operatorname{Ind}_{\left(U^{F} \cap\left(L_{I}\right)^{F}\right) \cdot t_{\sigma}}^{\left(L_{I}{ }^{F} \cdot t_{\sigma}\right.}\left(\operatorname{Res}_{\left(U^{F} \cap\left(L_{I}\right)^{F}\right) \cdot t_{\sigma}}^{U^{F} \cdot{ }^{t} \sigma}{ }^{(t} \widetilde{\chi}_{1}\right)\right) \times \operatorname{Id}_{V_{I}^{F}}\right]$, où on a choisi $t \in T\left(t \in T^{\sigma}\right.$ quand c'est possible et en particulier quand $\sigma$ est unipotent) tel que $\mathcal{L}(t) \in z$. Le calcul montre cependant que

$$
\begin{aligned}
& \operatorname{Ind}_{\left(U^{F} \cap\left(L_{I}\right)^{F}\right) \cdot t_{\sigma}}^{\left(L_{I}{ }^{F} \cdot t_{\sigma}\right.}\left(\operatorname{Res}_{\left(U^{F} \cap\left(L_{I}{ }^{F} \cdot t_{\sigma}{ }^{F}\right) \cdot t_{\sigma}\right.}\left({ }^{t} \widetilde{\chi}_{1}\right)\right) \times \operatorname{Id}_{V_{I}^{F}}
\end{aligned}
$$

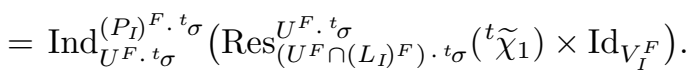

Ainsi, on obtient par la transitivité de l'induction :

$$
\mathrm{R}_{L_{I} \cdot \sigma}^{G \cdot \sigma}\left(\Gamma_{h_{\left(L_{I}\right)} \sigma(z)}^{L_{I} \cdot \sigma}\right)=\operatorname{Ind}_{U F \cdot t_{\sigma}}^{G^{F} \cdot{ }_{\sigma}}\left(\operatorname{Res}_{\left(U^{F} \cap\left(L_{I}\right)^{F}\right) \cdot t_{\sigma}}^{U^{F} \cdot{ }^{t}}\left({ }^{t} \widetilde{\chi}_{1}\right) \times \operatorname{Id}_{V_{I}^{F}}\right) .
$$

Donc

$$
\mathrm{D}_{G \cdot \sigma} \Gamma_{z}^{G \cdot \sigma}=\operatorname{Ind}_{U^{F} \cdot t_{\sigma}}^{G^{F}} \sum_{I \in \mathcal{P}(\Pi / \tau)^{\sigma}}(-1)^{|I / \sigma|}\left(\operatorname{Res}_{\left(U^{F} \cap\left(L_{I}\right)^{F}\right) \cdot t_{\sigma}}^{U^{F}}\left({ }^{t} \widetilde{\chi}_{1}\right) \times \operatorname{Id}_{V_{I}^{F}}\right) .
$$

On veut montrer que $\mathrm{D}_{G \cdot \sigma} \Gamma_{z}^{G \cdot \sigma}(g \cdot \sigma)$ est nul si $g \cdot \sigma$ n'est pas régulier unipotent rationnel quand $\sigma$ est unipotent et si $g \cdot \sigma$ n'est pas régulier pseudounipotent rationnel quand $\sigma$ est semi-simple. En fait, il suffit de prouver que

$$
\sum_{I \in \mathcal{P}(\Pi / \tau)^{\sigma}}(-1)^{|I / \sigma|}\left(\operatorname{Res}_{\left(U^{F} \cap \dot{t}_{\sigma}{ }^{U^{*}}\left(L_{I}\right)^{F}\right) \cdot t_{\sigma}}\left({ }^{t} \widetilde{\chi}_{1}\right) \times \operatorname{Id}_{V_{I}^{F}}\right)
$$

BULLETIN DE LA SOCIÉTÉ MATHÉMATIQUe DE FRANCE 
est nul en dehors des éléments réguliers unipotents (resp. pseudo-unipotents) de $U^{F} .{ }^{t} \sigma$. Soit $u \cdot{ }^{t} \sigma$ un élément de $U^{F} \cdot{ }^{t} \sigma$ et soit $\prod_{\mathcal{O} \in \Pi / \tau} u_{\mathcal{O}}$ la projection de $u$ $\operatorname{sur} \prod_{\mathcal{O} \in \Pi / \tau} U_{\mathcal{O}}$. On pose $\chi_{\mathcal{O}}=\left.{ }^{t} \chi_{1}\right|_{U_{\mathcal{O}}^{F}}$. On a alors

$$
\left({ }^{t} \widetilde{\chi}_{1}\right)\left(u \cdot{ }^{t} \sigma\right)=\prod_{\mathcal{O} \in \Pi / \tau} \chi_{\mathcal{O}}\left(u_{\mathcal{O}}\right) .
$$

Ainsi,

$$
\begin{array}{r}
\sum_{I \in \mathcal{P}(\Pi / \tau)^{\sigma}}(-1)^{|I / \sigma|}\left(\operatorname{Res}_{\left(U^{F}{ }^{F} \cap\left(L_{I}\right)^{F}\right) \cdot{ }^{t} \sigma}\left({ }^{t} \widetilde{\chi}_{1}\right) \times \operatorname{Id}_{V_{I}^{F}}\right)\left(u \cdot{ }^{t} \sigma\right) \\
=\sum_{I \in \mathcal{P}(\Pi / \tau)^{\sigma}}(-1)^{|I / \sigma|} \prod_{\mathcal{O} \in I} \chi_{\mathcal{O}}\left(u_{\mathcal{O}}\right) .
\end{array}
$$

On note à nouveau $\left\{\mathcal{O}_{1}, \ldots, \mathcal{O}_{s}\right\}$, les représentants des $\sigma$-orbites de $\Pi / \tau$.

Remarque 7.3. - Dans le cas où $t$ n'est pas $\sigma$-stable, les automorphismes $\sigma$ et ${ }^{t} \sigma$ agissent de la même façon sur les racines.

Donc $\left\{\mathcal{O}_{1}, \ldots, \mathcal{O}_{s}\right\}$ est aussi un ensemble de représentants des ${ }^{t} \sigma$-orbites de $\Pi / \tau$. Pour tout $k \in\{1, \ldots, s\}$, on note $o(k)$, l'ordre de $\mathcal{O}_{k}$ sous l'action de ${ }^{t} \sigma$. On a :

$$
\begin{aligned}
\left.\sum_{I \in \mathcal{P}(\Pi / \tau)^{\sigma}}(-1)^{|I / \sigma|}\left(\operatorname{Res}_{\left(U^{F} \cap\left(L_{I}\right)^{F}\right) \cdot{ }_{\sigma}}^{U^{F} \cdot{ }^{t} \sigma}{ }^{t} \widetilde{\chi}_{1}\right) \times \operatorname{Id}_{V_{I}^{F}}\right)\left(u \cdot{ }^{t} \sigma\right) \\
=\prod_{k=1}^{s}\left(1-\prod_{\ell=0}^{o(k)-1} \chi_{t \sigma^{\ell}\left(\mathcal{O}_{k}\right)}\left(u_{t_{\sigma^{\ell}}\left(\mathcal{O}_{k}\right)}\right)\right) .
\end{aligned}
$$

Le (i) de la proposition 7.2 se déduit donc de la proposition suivante.

Proposition 7.4. - Soit $\sigma^{\prime}$ un automorphisme quasi-central rationnel unipotent ou semi-simple de $G$. Soit $u=\prod_{\alpha \in \Phi^{+}} x_{\alpha}\left(k_{\alpha}\right)$ un élément de $U^{F}$ et soit $\chi$ un caractère régulier $\sigma^{\prime}$-stable de $U^{F}$. Alors, si $u \cdot \sigma^{\prime}$ n'est pas régulier, il existe une racine $\alpha$ de $\Pi$ telle que

$$
\chi\left(\prod_{i=0}^{o-1} u_{\sigma^{\prime i}(\alpha)}\left(k_{\sigma^{\prime i}(\alpha)}\right)\right)=1,
$$

où o est le cardinal de la $\sigma^{\prime}$-orbite de la $\tau$-orbite de $\alpha$ et où l'isomorphisme $\lambda \mapsto u_{\sigma^{\prime i}(\alpha)}$ est défini par la notation 4.11 .

Démonstration. - 1) Cas où $\sigma^{\prime}$ est unipotent. - Supposons $u \cdot \sigma^{\prime}$ non régulier. Alors, par le théorème 3.4 , il existe $\alpha \in \Pi$ tel que

$$
\sum_{i=0}^{n-1} c_{\sigma^{\prime i}(\alpha)} \cdots c_{\sigma^{\prime n-1}(\alpha)} k_{\sigma^{\prime i}(\alpha)}=0
$$

TOME $132-2004-\mathrm{N}^{\mathrm{O}} 2$ 
où $n$ est l'ordre de $\alpha$ sous l'action de $\sigma^{\prime}$. On note $\mathcal{A}$ la $\sigma^{\prime}$-orbite de $\alpha$ et $a$ l'ordre de $\mathcal{A}$ sous l'action de $\tau$. On note $\mathcal{O}$ la $\tau$-orbite de $\alpha$ et $o$ l'ordre de $\mathcal{O}$ sous l'action de $\sigma^{\prime}$.

Pour toute racine $\beta \in \Phi^{+}$, on a $k_{\tau(\beta)}=k_{\beta}^{q}$ car $u$ est rationnel et $c_{\tau(\beta)}=c_{\beta}^{q}$ par le lemme 4.5. Donc, pour tout $\ell \in\{1, \ldots, a-1\}$,

$$
\sum_{i=0}^{n-1} c_{\sigma^{\prime i}\left(\tau^{\ell}(\alpha)\right)} \cdots c_{\sigma^{\prime n-1}\left(\tau^{\ell}(\alpha)\right)} k_{\sigma^{\prime i}\left(\tau^{\ell}(\alpha)\right)}=\left(\sum_{i=0}^{n-1} c_{\sigma^{\prime i}(\alpha)} \cdots c_{\sigma^{\prime n-1}(\alpha)} k_{\sigma^{\prime i}(\alpha)}\right)^{q^{\ell}}=0
$$

On rappelle (lemme 4.7) que $\mathcal{A} \cup \cdots \cup \tau^{a-1}(\mathcal{A})=\mathcal{O} \cup \cdots \cup \sigma^{\prime o-1}(\mathcal{O})$. Ainsi, on peut trouver $u_{0}=\prod_{\beta \in \Phi^{+}} x_{\beta}\left(\ell_{\beta}\right) \in U^{F}$ tel que $\ell_{\beta}=0$ pour tout $\beta \in \bigcup_{\ell=0}^{a-1} \tau^{\ell}(\mathcal{A})$ et $v \in U$ tels que $u=v u_{0} \sigma^{\prime}(v)^{-1}$.

Pour toute $\tau$-orbite $\Omega$ de $\Pi$, on note $u_{\Omega}$ et $v_{\Omega}$ les projections respectives de $u$ et $v$ sur $\prod_{\beta \in \Omega} U_{\beta}$; alors on a

$$
\prod_{i=0}^{o-1} u_{\sigma^{\prime i}(\mathcal{O})}=\prod_{i=0}^{o-1} v_{\sigma^{\prime i}(\mathcal{O})} \prod_{i=0}^{o-1} \sigma^{\prime}\left(v_{\sigma^{\prime i}(\mathcal{O})}\right)^{-1} \quad \text { dans } U / U^{*}
$$

LEMME 7.5. - Si pour $\tilde{u} \in\left(U_{\mathcal{O}} \times \cdots \times U_{\sigma^{\prime o-1}(\mathcal{O})}\right)^{F}$ il existe $\tilde{v} \in U_{\mathcal{O}} \times \cdots \times$ $U_{\sigma^{\prime o-1}(\mathcal{O})}$ tel que $\tilde{u}=\tilde{v} \sigma^{\prime}(\tilde{v})^{-1}$, alors il existe $\tilde{w} \in\left(U_{\mathcal{O}} \times \cdots \times U_{\sigma^{\prime o-1}(\mathcal{O})}\right)^{F}$ tel que $\tilde{u}=\tilde{w} \sigma^{\prime}(\tilde{w})^{-1}$.

Démonstration. - Soient $\widetilde{U}=U_{\mathcal{O}} \times \cdots \times U_{\sigma^{\prime o-1}(\mathcal{O})}$ et $\mathcal{U}=\left\{\tilde{w} \sigma(\tilde{w})^{-1} ; \tilde{w} \in \widetilde{U}\right\}$. Alors $\mathcal{U}$ est l'orbite de 1 par $\sigma^{\prime}$-conjugaison dans $\widetilde{U}$. Selon Digne \& Michel [3, prop. 3.21] :

(i) $\tilde{w} \sigma^{\prime}(\tilde{w})^{-1}$ est rationnel si et seulement si $\tilde{w}^{-1} F(\tilde{w})$ appartient au stabilisateur de 1 dans $\widetilde{U}$ sous l'action de $\sigma^{\prime}$-conjugaison, c'est-à-dire à $\widetilde{U}^{\sigma^{\prime}}$;

(ii) les $\widetilde{U}^{F}$-orbites de $\sigma^{\prime}$-conjugaison de $\mathcal{U}^{F}$ sont paramétrées par les classes de $F$-conjugaison de $\widetilde{U}^{\sigma^{\prime}}$.

Cependant $\widetilde{U}^{\sigma^{\prime}}$ est un groupe abélien connexe; donc $\mathrm{H}^{1}\left(F, \widetilde{U}^{\sigma^{\prime}}\right)=1$, ce qui permet de conclure qu'il n'y a qu'une $\widetilde{U}^{F}$-orbite de $\sigma^{\prime}$-conjugaison dans $\mathcal{U}^{F}$. Ceci prouve le lemme 7.5 .

On a montré que si $u \cdot \sigma^{\prime}$ est unipotent rationnel non régulier, il existe une $\tau$-orbite $\mathcal{O}$ de $\Pi$ telle que si $o$ est l'ordre de $\mathcal{O}$ sous l'action de $\sigma^{\prime}$, alors on peut écrire

$$
u_{\mathcal{O}} \cdots u_{\sigma^{\prime o-1}(\mathcal{O})}=v_{\mathcal{O}} \cdots v_{\sigma^{\prime o-1}(\mathcal{O})} \sigma^{\prime}\left(v_{\mathcal{O}} \cdots v_{\sigma^{\prime o-1}(\mathcal{O})}\right)^{-1}
$$

BULLETIN DE LA SOCIÉTÉ MATHÉMATIQUE DE FRANCE 
où tous les facteurs du produit de droite sont rationnels. On a alors

$$
\begin{aligned}
\chi\left(\prod_{i=0}^{o-1} u_{\sigma^{\prime i}(\alpha)}\left(k_{\sigma^{\prime i}(\alpha)}\right)\right) & =\chi\left(\prod_{i=0}^{o-1} u_{\sigma^{\prime i}(\mathcal{O})}\right) \\
& =\chi\left(\prod_{i=0}^{o-1} v_{\sigma^{\prime i}(\mathcal{O})} \sigma\left(\prod_{i=0}^{o-1} v_{\sigma^{\prime i}(\mathcal{O})}\right)^{-1}\right) \\
& =\chi\left(\prod_{i=0}^{o-1} v_{\sigma^{\prime i}(\mathcal{O})}\right) \chi\left(\sigma\left(\prod_{i=0}^{o-1} v_{\sigma^{\prime i}(\mathcal{O})}\right)\right)^{-1} \\
& =\chi\left(\prod_{i=0}^{o-1} v_{\sigma^{\prime i}(\mathcal{O})}\right) \chi\left(\prod_{i=0}^{o-1} v_{\sigma^{\prime i}(\mathcal{O})}\right)^{-1}=1 .
\end{aligned}
$$

Ce qui prouve la proposition 7.4 lorsque $\sigma^{\prime}$ est unipotent.

2) Cas où $\sigma^{\prime}$ est semi-simple. - Soit us la décomposition de Jordan de $g \cdot \sigma^{\prime}$; alors $u$ et $s$ sont rationnels, la partie unipotente $u$ est dans $U^{F}$ et $s$ est un élément semi-simple rationnel de $U \cdot \sigma^{\prime}$. Donc, d'après le lemme 3.13 , on a $s \in \mathrm{Cl}_{U}\left(\sigma^{\prime}\right)^{F}$, c'est-à-dire $s \in \mathrm{Cl}_{U^{F}}\left(\sigma^{\prime}\right)$ car $U^{\sigma^{\prime}}$ est connexe. Ainsi, il existe $v \in U^{F}$ tel que $s={ }^{v} \sigma^{\prime}$ et $g=v u^{\prime} \sigma^{\prime}(v)^{-1}$, où on a posé $u^{\prime}={ }^{-1} u \in\left(U^{\sigma^{\prime}}\right)^{F}$.

Si on pose $u^{\prime}=\prod_{\alpha \in \Phi^{+}} x_{\alpha}\left(\ell_{\alpha}\right)$, alors, comme $\chi$ est $\sigma^{\prime}$-stable, pour toute racine simple $\alpha$, on a

$$
\chi\left(\prod_{i=0}^{o(\alpha)-1} u_{\sigma^{\prime i}(\alpha)}\left(k_{\sigma^{\prime i}(\alpha)}\right)\right)=\chi\left(\prod_{i=0}^{o(\alpha)-1} u_{\sigma^{\prime i}(\alpha)}\left(\ell_{\sigma^{\prime i}(\alpha)}\right)\right),
$$

où $o(\alpha)$ est le cardinal de la $\sigma^{\prime}$-orbite de la $\tau$-orbite de $\alpha$.

De plus, si $g \cdot \sigma^{\prime}$ n'est pas régulier dans $G \cdot \sigma^{\prime}$, alors $u^{\prime}$ ne l'est pas dans $\left(G^{\sigma^{\prime}}\right)^{0}$, donc il existe une $\sigma^{\prime}$-orbite $\mathcal{A}$ de $\Pi$ telle que pour toute racine $\alpha \in \mathcal{A}$ on ait $\ell_{\alpha}=0$. On choisit $\alpha \in \mathcal{A}$. Alors, pour tout $i \in\{1, \ldots, o(\alpha)-1\}$, où $o(\alpha)$ est le cardinal de la $\sigma^{\prime}$-orbite de la $\tau$-orbite de $\alpha$, on a $u_{\sigma^{\prime i}(\alpha)}\left(\ell_{\sigma^{\prime i}(\alpha)}\right)=1$, d'où

$$
\chi\left(\prod_{i=0}^{o(\alpha)-1} u_{\sigma^{\prime i}(\alpha)}\left(\ell_{\sigma^{\prime i}(\alpha)}\right)\right)=1 .
$$

Ceci termine les démonstrations de la proposition 7.4 et du (i) de la proposition 7.2.

Nous allons maintenant prouver le (ii) de la proposition 7.2. Soient $z$ et $z^{\prime}$ deux $F$-classes de $\mathrm{Z}\left(G^{\sigma}\right)$. Soient $t$ et $t^{\prime}$ dans $T$ tels que $\mathcal{L}(t) \in z$ et $\mathcal{L}\left(t^{\prime}\right) \in z^{\prime}$ (quand c'est possible, on choisit $t$ et $t^{\prime}$ dans $T^{\sigma}$ ). On pose

$$
\phi_{z^{\prime}}=\sum_{I \in \mathcal{P}(\Pi / \tau)^{\sigma}}(-1)^{|I / \sigma|}\left(\operatorname{Res}_{\left(U^{F} \cap\left(L_{I}\right)^{F}\right) \cdot t_{\sigma}^{\prime} \sigma}\left({ }^{t^{\prime}} \widetilde{\chi}_{1}\right) \times \operatorname{Id}_{V_{I}^{F}}\right) .
$$

TOME $132-2004-\mathrm{N}^{\mathrm{O}} 2$ 
On a alors,

$$
\mathrm{D}_{G \cdot \sigma} \Gamma_{z^{\prime}}^{G \cdot \sigma}=\operatorname{Ind}_{U^{F} \cdot t_{\sigma}^{\prime}}^{G^{F} \cdot t_{z^{\prime}}^{\prime}}
$$

Calculons le produit scalaire

$$
\begin{aligned}
& \left\langle\Gamma_{z}^{G \cdot \sigma}, \mathrm{D}_{G \cdot \sigma} \Gamma_{z^{\prime}}^{G \cdot \sigma}\right\rangle_{G^{F} \cdot \sigma}=\left\langle\operatorname{Ind}_{U^{F} \cdot{ }_{\sigma}{ }_{\sigma}}^{G^{F}{ }^{t}}\left({ }^{t} \widetilde{\chi}_{1}\right), \operatorname{Ind}_{U^{F} \cdot t^{\prime}{ }_{\sigma}}^{G^{F} \cdot t^{\prime}}\left(\phi_{z^{\prime}}\right)\right\rangle_{G^{F} \cdot \sigma} \\
& =\left\langle\operatorname{Res}_{U^{F} \cdot{ }^{\prime}{ }_{\sigma}}^{G^{F} \operatorname{tnd}^{\prime}} \operatorname{Ind}_{U^{F} \cdot t_{\sigma}}^{G^{F} \cdot{ }^{t}}\left({ }^{t} \widetilde{\chi}_{1}\right), \phi_{z^{\prime}}\right\rangle_{U^{F} \cdot t^{\prime} \sigma} .
\end{aligned}
$$

Supposons tout d'abord qu'il existe $t^{\prime \prime} \in T^{\sigma}$ tel que $\mathcal{L}\left(t^{\prime \prime}\right) \in z^{-1} z^{\prime}$ (c'est toujours vrai lorsque $\sigma$ est unipotent). Alors on peut supposer qu'on a choisi $t \in \mathcal{L}_{T}^{-1}(z)$ et $t^{\prime} \in \mathcal{L}_{T}^{-1}\left(z^{\prime}\right)$ tels que $t^{-1} t^{\prime} \in T^{\sigma}$. On a ${ }^{t^{\prime}} \sigma={ }^{t} \sigma$, d'où

$$
\left\langle\Gamma_{z}^{G \cdot \sigma}, \mathrm{D}_{G \cdot \sigma} \Gamma_{z^{\prime}}^{G \cdot \sigma}\right\rangle_{G^{F} \cdot \sigma}=\left\langle\operatorname{Res}_{U^{F} \cdot t_{\sigma}}^{G^{F} \cdot{ }^{t}} \operatorname{Ind}_{U^{F} \cdot t_{\sigma}}^{G^{F} \cdot{ }^{t}}\left({ }^{t} \widetilde{\chi}_{1}\right), \phi_{z^{\prime}}\right\rangle_{U^{F} \cdot t_{\sigma}} \cdot
$$

Par la formule de Mackey pour les fonctions de ${ }^{t} \sigma$-classes et en utilisant la bijection entre $U^{F} \backslash G^{F} / U^{F}$ et $\mathrm{N}_{G}(T)^{F}$, on obtient

$$
\left\langle\Gamma_{z}^{G \cdot \sigma}, \mathrm{D}_{G \cdot \sigma} \Gamma_{z^{\prime}}^{G \cdot \sigma}\right\rangle_{G^{F} \cdot \sigma}=\sum_{n \in\left(\mathrm{N}_{G}(T)^{F}\right)^{t_{\sigma}}}\left\langle{ }^{n}\left({ }^{t} \widetilde{\chi}_{1}\right), \phi_{z^{\prime}}\right\rangle_{\left(U^{F} \cap{ }^{n} U^{F}\right) \cdot{ }_{\sigma}} \cdot
$$

Lorsque $\sigma$ est unipotent (resp. semi-simple), le fait qu'un élément régulier unipotent (resp. pseudo-unipotent) ne normalise qu'un seul sous-groupe de Borel (propositions 3.3 et 3.16) et le fait que $n$ soit ${ }^{t} \sigma$-stable entraînent que $\left(U^{F} \cap{ }^{n} U^{F}\right) \cdot{ }^{t} \sigma$ ne contient pas d'éléments réguliers si $n$ n'est pas dans $\left(T^{t} \sigma\right)^{F}$.

Par ailleurs, $\left.\left\langle\left({ }^{t} \widetilde{\chi}_{1}\right), \operatorname{Res}_{\left(U^{F} \cap\left(L_{I}\right)\right.}^{\left.U^{F}\right) \cdot{ }^{t}{ }_{\sigma}}\left({ }^{t} \widetilde{\chi}_{1}\right) \times \operatorname{Id}_{V_{I}^{F}}\right)\right\rangle_{U^{F} \cdot t_{\sigma}}$ est nul si $I \neq \Pi / \tau$ $\left(\operatorname{car}\left\langle\left({ }^{t} \widetilde{\chi}_{1}\right), \operatorname{Id}_{V_{I}^{F}}\right\rangle_{V_{I}^{F}}\right.$ est nul si $\left.V_{I}^{F} \neq\{1\}\right)$. On a donc

$$
\left\langle\Gamma_{z}^{G \cdot \sigma}, \mathrm{D}_{G \cdot \sigma} \Gamma_{z^{\prime}}^{G \cdot \sigma}\right\rangle_{G^{F} \cdot \sigma}=(-1)^{|\Pi / \tau \times \sigma|} \sum_{t^{\prime \prime} \in\left(T^{t_{\sigma}}\right)^{F}}\left\langle{ }^{\prime \prime}{ }^{\prime} \chi_{1},{ }^{t^{\prime}} \chi_{1}\right\rangle_{U^{F}}
$$

Cependant, comme $t^{-1} t^{\prime}$ est $\sigma$-stable, les caractères $t^{\prime \prime} t \chi_{1}$ et ${ }^{t^{\prime}} \chi_{1}$ ne peuvent être égaux que si $t^{-1} t^{\prime} \in\left(T^{\sigma}\right)^{F}$ (c'est-à-dire si $z=z^{\prime}$ ), et si $t^{\prime \prime} \in t^{-1} t^{\prime} \mathrm{Z}\left(G^{\sigma}\right)^{F}$.

Supposons maintenant qu'il n'existe pas de $t^{\prime \prime}$ dans $T^{\sigma}$ tel que $\mathcal{L}\left(t^{\prime \prime}\right) \in z^{-1} z^{\prime}$.

LEMME 7.6. - Sous l'hypothèse précédente, pour tout $u \in U^{F}$ tel que $u \cdot{ }^{\prime} \sigma$ soit régulier, on a $\operatorname{Ind}_{U^{F} \cdot t_{\sigma}}^{G_{\sigma}^{F}}\left({ }^{t} \widetilde{\chi}_{1}\right)\left(u \cdot{ }^{\prime} \sigma\right)=0$.

BULLETIN DE LA SOCIÉtÉ MATHÉMATIQUE DE FRANCE 
Démonstration. - On pose $t_{0}=t^{-1} t^{\prime}$. On a

$$
\begin{aligned}
& \operatorname{Ind}_{U F \cdot{ }_{\sigma}}^{G^{F} \cdot{ }^{t}}\left({ }^{t} \widetilde{\chi}_{1}\right)\left(u \cdot{ }^{t^{\prime}} \sigma\right) \\
& =1 /\left|U^{F}\right| \sum_{\left\{x \in G^{F} \mid\left(x \cdot{ }^{t} \sigma\right)\left(u \cdot{ }^{\prime} \sigma\right)\left(x \cdot{ }^{t} \sigma\right)^{-1} \in U^{F} \cdot{ }^{t} \sigma\right\}}\left({ }^{t} \widetilde{\chi}_{1}\right)\left(\left(x \cdot{ }^{t} \sigma\right)\left(u \cdot{ }^{t^{\prime}} \sigma\right)\left(x \cdot{ }^{t} \sigma\right)^{-1}\right) \\
& =1 /\left|U^{F}\right| \sum_{\left\{x \in G^{F} \mid x\left({ }^{t} \sigma\right) u t_{0} \sigma\left(t_{0}\right)^{-1} x^{-1} \in U^{F} \cdot{ }^{t} \sigma\right\}}\left({ }^{t} \widetilde{\chi}_{1}\right)\left(x\left({ }^{t} \sigma\right) u t_{0} \sigma\left(t_{0}\right)^{-1} x^{-1}\right) \\
& =1 /\left|U^{F}\right| \sum_{\left\{x \in G^{F} \mid\left(x \sigma\left(t_{0}\right)\right)^{t} \sigma\left({ }^{t_{0}^{-1}} u\right)^{t} \sigma\left(x \sigma\left(t_{0}\right)\right)^{-1} \in U^{F}\right\}}\left({ }^{t} \widetilde{\chi}_{1}\right)\left(\left(x \sigma\left(t_{0}\right)\right)^{t} \sigma\left({ }^{t_{0}^{-1}} u \cdot{ }^{t} \sigma\right)\left(x \sigma\left(t_{0}\right)\right)^{-1}\right) \\
& =1 /\left|U^{F}\right| \sum_{\left\{y \in G^{F} \mid\left(y t_{0}\right)\left({ }^{t-1} u \cdot{ }^{t} \sigma\right)\left(y t_{0}\right)^{-1} \in U^{F} \cdot{ }^{t} \sigma\right\}}\left({ }^{t} \widetilde{\chi}_{1}\right)\left(\left(y t_{0}\right)\left(\left({ }^{t_{0}^{-1}} u\right) \cdot{ }^{t} \sigma\right)\left(y t_{0}\right)^{-1}\right) .
\end{aligned}
$$

On considère l'ensemble suivant :

$$
\mathcal{E}=\left\{y \in G^{F} ;\left(y t_{0}\right)\left(\left({ }^{t_{0}^{-1}} u\right) \cdot{ }^{t} \sigma\right)\left(y t_{0}\right)^{-1} \in U^{F} \cdot{ }^{t} \sigma\right\} .
$$

Pour montrer que $\operatorname{Ind}_{U^{F} \cdot{ }^{t}{ }_{\sigma}}^{G^{F}}\left({ }^{t} \widetilde{\chi}_{1}\right)\left(u \cdot{ }^{t^{\prime}} \sigma\right)=0$, il suffit de montrer que $\mathcal{E}$ est vide. Le fait que $u \cdot{ }^{\prime}{ }_{\sigma}$ soit régulier entraîne que ${ }^{t_{0}^{-1}} u \cdot{ }^{t} \sigma$ est régulier aussi. Soit $y \in \mathcal{E}$, alors $y t_{0}\left({ }^{t_{0}^{-1}} u \cdot{ }^{t} \sigma\right) \in \mathrm{N}_{\widetilde{G}}(B)$, donc ${ }^{t_{0}^{-1}} u \cdot{ }^{t} \sigma \in \mathrm{N}_{\widetilde{G}}\left({ }^{t_{0}^{-1}} y^{-1} B\right)$, ce qui entraîne que $y t_{0} \in B$ car $\left({ }^{t_{0}^{-1}} u\right) \cdot{ }^{t} \sigma$ est régulier et donc d'après les propositions 3.3 et 3.16 , ne normalise qu'un seul sous-groupe de Borel de $G$. On pose $y=v t_{1}$ avec $v \in U$ et $t_{1} \in T$; alors il existe $t_{2} \in T^{\sigma}$ tel que $t_{1}=t_{2} t_{0}$ et $t_{1} \in T^{F}$. Ainsi $t_{2} F\left(t_{2}\right)^{-1}=t_{0}^{-1} F\left(t_{0}\right) \in z^{-1} z^{\prime}$, donc $z^{-1} z^{\prime} \cap \mathcal{L}_{T^{\sigma}}\left(T^{\sigma}\right)$ n'est pas vide ce qui contredit l'hypothèse faite sur $z^{-1} z^{\prime}$. Donc $\mathcal{E}$ est vide et on obtient le résultat du lemme 7.6.

D'après le lemme précédent, on a $\operatorname{Ind}_{U^{F} \cdot t_{\sigma}}^{G^{F} \cdot{ }^{t}}\left({ }^{t} \widetilde{\chi}_{1}\right)\left(u \cdot{ }^{t} \sigma\right)=0$ pour tout $u \in U^{F}$ tel que $u \cdot{ }^{\prime} \sigma$ soit régulier, et d'après la démonstration du (i), $\phi_{z^{\prime}}\left(u \cdot{ }^{\prime} \sigma\right)=0$ pour tout $u \in U^{F}$ tel que $u \cdot{ }^{t^{\prime}} \sigma$ ne soit pas régulier. Donc, lorsqu'il n'existe pas de $t^{\prime \prime} \in T^{\sigma}$ tel que $\mathcal{L}\left(t^{\prime \prime}\right) \in z^{-1} z^{\prime}$, on a bien $\left\langle\Gamma_{z}^{G \cdot \sigma}, \mathrm{D}_{G \cdot \sigma} \Gamma_{z^{\prime}}^{G \cdot \sigma}\right\rangle_{G^{F} \cdot \sigma}=0$, ce qui termine la preuve de la proposition 7.2 .

\section{Valeurs des fonctions de $\sigma$-classes sur les éléments réguliers unipotents ou pseudo-unipotents}

On suppose que $\sigma$ est rationnel quasi-central unipotent ou semi-simple. On choisit $u_{0} \in U^{F}$ tel que $u_{0} \cdot \sigma$ soit régulier. Soit $\mathcal{Z}$ l'ensemble des éléments $z$ de $\mathrm{H}^{1}\left(F, \mathrm{Z}\left(G^{\sigma}\right)\right)$ tels qu'il existe $t \in T^{\sigma}$ pour lequel $t^{-1} F(t) \in z$. Si $\sigma$ est unipotent, on a $\mathcal{Z}=\mathrm{H}^{1}\left(F, \mathrm{Z}\left(G^{\sigma}\right)\right)$.

Pour tout $z \in \mathrm{H}^{1}\left(F, \mathrm{Z}\left(G^{\sigma}\right)\right)$, soit $t \in T$ tel que $t^{-1} F(t) \in z$. On définit l'ensemble $\mathcal{U}_{z}=\left\{x \cdot \sigma \in G^{F} \cdot \sigma ; x \cdot \sigma \sim_{G^{F}}{ }^{t}\left(u_{0} U^{*} \cdot \sigma\right)\right\}$, définition qui est indépendante du choix de $t$.

TOME $132-2004-\mathrm{N}^{\mathrm{O}} 2$ 
Proposition 8.1. - (i) Les ensembles $\mathcal{U}_{z}$ sont disjoints.

(ii) La réunion des $\mathcal{U}_{z}$ est l'ensemble des éléments réguliers unipotents (resp. pseudo-unipotents) de $G^{F} \cdot \sigma$ lorsque $\sigma$ est unipotent (resp. semi-simple).

(iii) Si $\sigma$ est semi-simple et si la caractéristique est bonne pour $\left(G^{\sigma}\right)^{0}$, alors chaque $\mathcal{U}_{z}$ est une classe de conjugaison sous $G^{F}$.

Démonstration. - (i) Soient $z$ et $z^{\prime}$ dans $\mathrm{H}^{1}\left(F, \mathrm{Z}\left(G^{\sigma}\right)\right)$ et $t \in T$ tel que $\mathcal{L}(t)$ soit un représentant de $z$. Alors, on a $t \mathcal{U}_{z^{\prime}} t^{-1}=\mathcal{U}_{z z^{\prime}}$. Il suffit donc de montrer que $\mathcal{U}_{z} \cap \mathcal{U}_{1}$ est vide pour tout $z \in \mathrm{H}^{1}\left(F, \mathrm{Z}\left(G^{\sigma}\right)\right)$.

Soit $z \in \mathrm{H}^{1}\left(F, \mathrm{Z}\left(G^{\sigma}\right)\right)$ tel que $\mathcal{U}_{z} \cap \mathcal{U}_{1}$ soit non vide. Soient $t \in \mathcal{L}_{T}^{-1}(z)$, $x \in G^{F}$ et $u^{*}, v^{*} \in\left(\left(U^{\sigma}\right)^{*}\right)^{F}$ tels que

$$
{ }^{t}\left(u_{0} u^{*} \cdot \sigma\right)={ }^{x}\left(u_{0} v^{*} \cdot \sigma\right)
$$

Comme $u_{0} u^{*} \cdot \sigma$ et $u_{0} v^{*} \cdot \sigma$ sont réguliers, ils ne normalisent qu'un seul sous-groupe de Borel qui est $B$ (propositions 3.3 et 3.16); donc ${ }^{t}\left(u_{0} u^{*} \cdot \sigma\right.$ ) et ${ }^{x}\left(u_{0} v^{*} \cdot \sigma\right)$ normalisent le même sous-groupe de Borel qui est encore $B$, ce qui entraîne que $x \in B^{F}$. On a donc $x=s u_{x}$ avec $s \in T^{F}$ et $u_{x} \in U^{F}$. L'équation (2) entraîne que $t^{-1} s\left(u_{0} u^{*} \cdot \sigma\right) \in U^{F} \cdot \sigma$, donc que $\left(t^{-1} s\right)^{-1} \sigma\left(t^{-1} s\right)=1$, donc l'existence de $t_{0} \in T^{\sigma}$ tel que $s=t t_{0}$. Ainsi $t_{0} F\left(t_{0}\right)^{-1}=t^{-1} F(t) \in z$ et comme $t_{0} \in T^{\sigma}$, on a $z \in \mathcal{Z}$. Donc on peut supposer que $t \in T^{\sigma}, s$ est aussi $\sigma$-stable. Donc d'après (2), on obtient ${ }^{-1} t u_{0} u_{1}^{*}=u_{x} u_{0} \sigma\left(u_{x}\right)^{-1} u_{2}^{*}$ avec $u_{i}^{*} \in U^{*}$. On note $\widetilde{B}=B / U^{*}$. Si on note respectivement $\tilde{u}_{x}$ et $\tilde{u}_{0}$ les projections de $u_{x}$ et $u_{0}$ dans $\widetilde{B}$, alors on obtient $\left(\tilde{u}_{x} s t^{-1}\right) \tilde{u}_{0} \sigma\left(\tilde{u}_{x} s t^{-1}\right)^{-1}=\tilde{u}_{0}$ dans $\widetilde{B}$. Ce qui entraîne que $s t^{-1} \in \mathrm{Z}\left(G^{\sigma}\right)$ : c'est clair d'après le lemme 3.11 lorsque $\sigma$ est unipotent, c'est encore vrai lorsque $\sigma$ est semi-simple car dans ce cas $u_{0} \cdot \sigma$ est une décomposition de Jordan et $u_{0}$ est régulier dans $\left(G^{\sigma}\right)^{0}$ ce qui entraine que $\mathrm{C}_{G}\left(u_{0} \cdot \sigma\right)=\mathrm{Z}\left(G^{\sigma}\right) \times \mathrm{C}_{U^{\sigma}}\left(u_{0}\right)$. Donc il existe $\tau \in \mathrm{Z}\left(G^{\sigma}\right)$ tel que $\tau^{-1} F(\tau)=t^{-1} F(t) \in z$ et on a $z=1$.

(ii) Cas unipotent. - Soit $x \cdot \sigma$ un élément unipotent régulier rationnel, alors $x \cdot \sigma$ normalise un unique sous-groupe de Borel $B^{\prime}$. Par ailleurs, ${ }^{x} \cdot \sigma F\left(B^{\prime}\right)=$ $F\left({ }^{x \cdot \sigma} B^{\prime}\right)=F\left(B^{\prime}\right)$, donc $x \cdot \sigma$ normalise $F\left(B^{\prime}\right)$, ce qui signifie que $B^{\prime}$ est rationnel. Ainsi, il existe $g \in G^{F}$ tel que $B^{\prime}={ }^{g} B$ et $g^{-1} x \sigma(g) \cdot \sigma=b \cdot \sigma$ est un élément unipotent régulier de $B \cdot \sigma$.

Or, d'après le lemme $3.5, b \cdot \sigma$ est régulier si et seulement si son image $\tilde{b}$ par la projection $B \rightarrow \widetilde{B}$ est $\sigma$-conjugué sous $\widetilde{B}$ à $\tilde{u}_{0}$. On note $\mathcal{U}$ la classe de $\sigma$-conjugaison de $\tilde{u}_{0}$ sous $\widetilde{B}$. Les classes de $\sigma$-conjugaison de $\mathcal{U}^{F}$ dans $\widetilde{B}^{F}$ sont paramétrées par $\mathrm{H}^{1}\left(F, \mathrm{Z}\left(G^{\sigma}\right)\right)$. Donc, si $\tilde{b} \in \mathcal{U}^{F}$, il existe $z \in \mathrm{H}^{1}\left(F, \mathrm{Z}\left(G^{\sigma}\right)\right)$ et $t$ dans $\mathcal{L}_{T^{\sigma}}^{-1}\left(\mathrm{Z}\left(G^{\sigma}\right)\right)$, un représentant de $z$, tels que $\tilde{b}$ soit dans la classe de $\sigma$-conjugaison sous $\widetilde{B}^{F}$ de ${ }^{t} \tilde{u}_{0}$. Soit $\tilde{w} \in \widetilde{B}^{F}$ tel que $\tilde{b}=\tilde{w}^{t} \tilde{u}_{0} \sigma(\tilde{w})^{-1}$. Soit $w \in B^{F}$ qui ait pour image $\tilde{w}$ dans $\widetilde{B}^{F}$. Alors il existe $u^{*} \in\left(U^{*}\right)^{F}$ tel que $b=w^{t}\left(u_{0} u^{*}\right) \sigma(w)^{-1}$. Donc $b \cdot \sigma$ et par conséquent $x \cdot \sigma$ sont dans $\mathcal{U}_{z}$. 
Cas semi-simple. - Soit $x \cdot \sigma$ un élément pseudo-unipotent régulier rationnel; alors, par définition, il existe $g \in G$ tel que $x \cdot \sigma={ }^{g}\left(u_{0} \cdot \sigma\right)$ et tel que $g^{-1} F(g) \in$ $\mathrm{C}_{G}\left(u_{0} \cdot \sigma\right)$; on a $\mathrm{C}_{G}\left(u_{0} \cdot \sigma\right)=\mathrm{Z}\left(G^{\sigma}\right) \times \mathrm{C}_{U^{\sigma}}\left(u_{0}\right)$. Comme $T$ et $U^{\sigma}$ sont connexes, $\mathcal{L}_{T}$ et $\mathcal{L}_{U^{\sigma}}$ sont surjectives. Donc il existe $t \in T, u \in U^{\sigma}$ et $h \in G^{F}$ tels que $g=h t u$. Ainsi $x \cdot \sigma={ }^{h}\left({ }^{t}\left(u_{0}\left[u_{0}^{-1}, u\right] \cdot \sigma\right)\right)$ et $\left[u_{0}^{-1}, u\right] \in\left(U^{\sigma}\right)^{*}$, donc $x \cdot \sigma \in \mathcal{U}_{z}$, où $z$ est tel que $t^{-1} F(t) \in z$.

La réciproque est claire car pour tout $u^{*} \in\left(U^{\sigma}\right)^{*}, u_{0} u^{*}$ est régulier dans $\left(G^{\sigma}\right)^{0}$ (voir [3, prop. 14.14]), et comme dans $\left(G^{\sigma}\right)^{0}$ tous les éléments unipotents réguliers sont conjugués (voir [3, prop. 14.16]), il existe $g \in\left(G^{\sigma}\right)^{0}$ tel que $u_{0} u^{*}=$ ${ }^{g} u_{0}$. Ainsi $u_{0} u^{*} \cdot \sigma={ }^{g}\left(u_{0} \cdot \sigma\right)$, donc $u_{0} u^{*} \cdot \sigma$ est régulier pseudo-unipotent.

(iii) D'après la proposition 3.15, les éléments pseudo-unipotents réguliers sont les conjugués sous $G$ de $u_{0} \cdot \sigma$. Ainsi les $G^{F}$-classes d'éléments pseudounipotents réguliers rationnels sont paramétrées par $\mathrm{H}^{1}\left(F, A\left(u_{0} \cdot \sigma\right)\right)$, où on note $A\left(u_{0} \cdot \sigma\right)=\mathrm{C}_{G}\left(u_{0} \cdot \sigma\right) / \mathrm{C}_{G}\left(u_{0} \cdot \sigma\right)^{0}$. On rappelle que, comme $\sigma$ est semisimple, on a $\mathrm{C}_{G}\left(u_{0} \cdot \sigma\right)=\mathrm{Z}\left(G^{\sigma}\right) \times \mathrm{C}_{U^{\sigma}}\left(u_{0}\right)$. De plus, si la caractéristique est bonne pour $\left(G^{\sigma}\right)^{0}$, alors $\mathrm{C}_{U^{\sigma}}\left(u_{0}\right)$ est connexe (voir [3, prop. 14.18]). Ainsi $\mathrm{H}^{1}\left(F, A\left(u_{0} \cdot \sigma\right)\right)=\mathrm{H}^{1}\left(F, \mathrm{Z}\left(G^{\sigma}\right)\right)$. Le nombre de classes pseudo-unipotentes régulières dans $G^{F}$ est donc $\left|\mathrm{H}^{1}\left(F, \mathrm{Z}\left(G^{\sigma}\right)\right)\right|$. Comme les $\mathcal{U}_{z}$ sont des réunions de telles classes et qu'ils sont disjoints, on en déduit le résultat.

DÉfinition 8.2. - Soit $c$ une réunion de classes de conjugaison de $G^{F} \cdot \sigma$; la fonction de classe $\gamma_{c}$ est définie sur $G^{F} \cdot \sigma$ par

$$
\gamma_{c}(x)= \begin{cases}\left|G^{F}\right| /|c| & \text { si } x \in c, \\ 0 & \text { sinon. }\end{cases}
$$

DÉfinition 8.3. - Pour tout $z \in \mathcal{Z}$, on note $\Phi_{z}$ la $\left(T^{\sigma}\right)^{F}$-orbite de ${ }^{t} \chi_{1}$, où $t \in T^{\sigma}$ est tel que $t^{-1} F(t) \in z$ et on pose

$$
\sigma_{z}=\sum_{\chi \in \Phi_{z}} \tilde{\chi}\left(u_{0} \cdot \sigma\right)
$$

La définition ci-dessus généralise celle des coefficients $\sigma_{z}$ qui ont été définis dans [2, déf. 3.4] et dans [3, déf. 14.34] dans le cadre des groupes réductifs connexes. Les coefficients $\sigma_{z}$ de $G \cdot \sigma$ peuvent s'exprimer en fonction des coefficients $\sigma_{z}$ analogues pour le groupe réductif connexe $\left(G^{\sigma}\right)^{0}$.

THÉORÈME 8.4. - (i) Soit $\widetilde{\chi}$ un caractère de $\widetilde{G}^{F}$ dont la restriction à $G^{F}$ est irréductible et soit $z \in \mathrm{H}^{1}\left(F, \mathrm{Z}\left(G^{\sigma}\right)\right)$; alors, avec les notations ci-dessus,

$$
\begin{aligned}
& \frac{1}{\left|\mathcal{U}_{z}\right|} \sum_{u \cdot \sigma \in \mathcal{U}_{z}} \tilde{\chi}(u \cdot \sigma) \\
& =(-1)^{|(\Pi / \sigma) / \tau|} \sum_{\left\{z^{\prime} \in \mathrm{H}^{1}\left(F, \mathrm{Z}\left(G^{\sigma}\right)\right) ; z^{-1} z^{\prime} \in \mathcal{Z}\right\}} \sigma_{z^{-1} z^{\prime}}\left\langle\mathrm{D}_{G \cdot \sigma}(\tilde{\chi}), \Gamma_{z^{\prime}}^{G \cdot \sigma}\right\rangle_{G^{F} \cdot \sigma} .
\end{aligned}
$$

TOME $132-2004-\mathrm{N}^{\mathrm{O}} 2$ 
(ii) On a $\left\langle\sum_{z} \Gamma_{z}^{G \cdot \sigma} ; \sum_{z} \Gamma_{z}^{G \cdot \sigma}\right\rangle_{G F \cdot \sigma}=\left|\mathrm{H}^{1}\left(F, \mathrm{Z}\left(G^{\sigma}\right)\right)\right| \cdot\left|\mathrm{Z}\left(G^{\sigma}\right)^{F}\right| q^{\ell}$, où $\ell$ est le rang semi-simple de $\left(G^{\sigma}\right)^{0}$ et $z$ parcourt $\mathrm{H}^{1}\left(F, \mathrm{Z}\left(G^{\sigma}\right)\right)$.

Démonstration. - (i) Soit $t \in \mathcal{L}_{T}^{-1}(z)$ (si c'est possible et en particulier lorsque $\sigma$ est unipotent, on choisit $t \in T^{\sigma}$ ). On a vu dans la démonstration de la proposition 7.2 que $\mathrm{D}_{G \cdot \sigma} \Gamma_{z}^{G \cdot \sigma}=\operatorname{Ind}_{U^{F} \cdot{ }_{\sigma}}^{G^{F}{ }^{t}} \phi_{z}$, où $\phi_{z}$ est une fonction de classe de $U^{F} \cdot{ }^{t} \sigma$ nulle en dehors des éléments réguliers. Plus précisément, on a vu que si $u \cdot{ }^{t} \sigma \in U^{F} \cdot{ }^{t} \sigma$ est régulier, si on note $\prod_{\mathcal{O} \in \Pi / \tau} u_{\mathcal{O}}$ la projection de $u$ sur $\prod_{\mathcal{O} \in \Pi / \tau} U_{\mathcal{O}}$ et si on note $\left\{\mathcal{O}_{1}, \ldots, \mathcal{O}_{s}\right\}$, les représentants des $\sigma$-orbites de $\Pi / \tau$ et pour tout $k \in\{1, \ldots, s\}, o(k)$, l'ordre de $\mathcal{O}_{k}$ sous l'action de $\sigma$ (rappelons que ${ }^{t} \sigma$ et $\sigma$ agissent de la même façon sur les racines), alors

$$
\phi_{z}\left(u \cdot{ }^{t} \sigma\right)=\prod_{k=1}^{s-1}\left(1-\prod_{\ell=0}^{o(k)-1} \chi_{\sigma^{\ell}\left(\mathcal{O}_{k}\right)}\left(u_{\sigma^{\ell}\left(\mathcal{O}_{k}\right)}\right)\right) .
$$

Ainsi, $\phi_{z}$ est l'extension normalisée à $U^{F} \cdot\left\langle{ }^{t} \sigma\right\rangle$ d'une fonction de classe ${ }^{t} \sigma$-stable $\operatorname{sur} U^{F}$, triviale sur $\left(U^{*}\right)^{F}$. Nous allons montrer que cette propriété de $\phi_{z}$ entraîne que $\mathrm{D}_{G \cdot \sigma} \Gamma_{z}^{G \cdot \sigma}=\operatorname{Ind}_{U^{F} \cdot t_{\sigma}}^{G^{F} \phi_{z}}$ est constante sur chaque $\mathcal{U}_{z^{\prime}}$.

Soit $z^{\prime} \in \mathrm{H}^{1}\left(F, \mathrm{Z}\left(G^{\sigma}\right)\right)$, soit $t^{\prime} \in \mathcal{L}_{T}^{-1}\left(\mathrm{Z}\left(G^{\sigma}\right)\right)$ un représentant de $z^{\prime}$, soient $x \cdot \sigma$ et $x^{\prime} \cdot \sigma$ dans $\mathcal{U}_{z^{\prime}}$. Alors il existe $u_{*}$ et $u_{*}^{\prime}$ dans $\left(\left(U^{\sigma}\right)^{*}\right)^{F}$ tels que $x \cdot \sigma$ et $x^{\prime} \cdot \sigma$ soient conjugués sous $G^{F}$ respectivement à ${ }^{\prime}\left(u_{0} u_{*} \cdot \sigma\right)$ et ${ }^{\prime}\left(u_{0} u_{*}^{\prime} \cdot \sigma\right)$. On a alors

$$
\begin{aligned}
\mathrm{D}_{G \cdot \sigma} \Gamma_{z}^{G \cdot \sigma}\left(x^{\prime} \cdot \sigma\right) & =\operatorname{Ind}_{U^{F} \cdot{ }_{\sigma}}^{G^{F} \cdot{ }_{\sigma}} \phi_{z}\left({ }^{\prime}\left(u_{0} u_{*}^{\prime} \cdot \sigma\right)\right) \\
& =\frac{1}{\left|U^{F}\right|} \sum_{x \in \mathcal{E}} \phi_{z}\left(x^{t} \sigma t^{\prime} t^{-1}\left({ }^{t}\left(u_{0} u_{*}^{\prime}\right) \cdot{ }^{t} \sigma\right)\left(x^{t} \sigma t^{\prime} t^{-1}\right)^{-1}\right),
\end{aligned}
$$

où $\mathcal{E}$ est défini par $\mathcal{E}=\left\{x \in G^{F} ; x^{t} \sigma t^{\prime} t^{-1}\left({ }^{t}\left(u_{0} u_{*}^{\prime}\right) \cdot{ }^{t} \sigma\right)\left(x^{t} \sigma t^{\prime} t^{-1}\right)^{-1} \in U^{F} \cdot{ }^{t} \sigma\right\}$. Cependant ${ }^{t}\left(u_{0} u_{*}^{\prime}\right) \cdot{ }^{t} \sigma$ est un élément régulier du quasi-borel $\mathrm{N}_{\widetilde{G}}(B)$ et $\mathrm{N}_{\widetilde{G}}(B)$ est l'unique quasi-borel qui le contient. Si $x$ est un élément de $\mathcal{E}$, on a

$$
x^{t} \sigma t^{\prime} t^{-1}\left({ }^{t}\left(u_{0} u_{*}^{\prime}\right) \cdot{ }^{t} \sigma\right)\left(x^{t} \sigma t^{\prime} t^{-1}\right)^{-1} \in \mathrm{N}_{\widetilde{G}}(B) .
$$

Cela entraîne que $x^{t} \sigma t^{\prime} t^{-1}$ est aussi dans $\mathrm{N}_{\widetilde{G}}(B)$ et que $x \in B^{F}$. On peut ainsi écrire $x^{t} \sigma t^{\prime} t^{-1}=v y$ avec $v \in U^{F}$ et $y \in T^{t}{ }^{\sigma} \cdot{ }^{t} \sigma$. On obtient alors

$$
v y\left({ }^{t}\left(u_{0} u_{*}^{\prime}\right) \cdot{ }^{t} \sigma\right)(v y)^{-1}=v y^{t} u y^{-1 t} \sigma(v)^{-1} w \cdot{ }^{t} \sigma
$$

avec $w \in U^{*}$. Mais comme $\phi_{z}$ est une extension à $U^{F} \cdot\langle\sigma\rangle$ d'une fonction de classe ${ }^{t} \sigma$-stable de $U^{F}$ triviale sur $\left(U^{*}\right)^{F}, \phi_{z}\left(v y^{t} u y^{-1 t} \sigma(v)^{-1} w \cdot{ }^{t} \sigma\right)$ ne dépend pas de $w$ et $\phi_{z}\left(x^{t} \sigma t^{\prime} t^{-1}\left({ }^{t}\left(u_{0} u_{*}^{\prime}\right) \cdot{ }^{t} \sigma\right)\left(x^{t} \sigma t^{\prime} t^{-1}\right)^{-1}\right)$ ne dépend pas de $u_{*}^{\prime}$. On a donc

$$
\begin{aligned}
\mathrm{D}_{G \cdot \sigma} \Gamma_{z}^{G \cdot \sigma}\left(x^{\prime} \cdot \sigma\right) & =\operatorname{Ind}_{U^{F} \cdot{ }_{\sigma}}^{G_{\sigma}^{F}} \phi_{z}\left({ }^{\prime}\left(u_{0} u_{*}^{\prime} \cdot \sigma\right)\right) \\
& =\operatorname{Ind}_{U^{F} \cdot{ }_{\sigma}{ }_{\sigma}}^{G} \phi_{z}\left({ }^{t^{\prime}}\left(u_{0} u_{*} \cdot \sigma\right)\right)=\mathrm{D}_{G \cdot \sigma} \Gamma_{z}^{G \cdot \sigma}(x \cdot \sigma) .
\end{aligned}
$$

BULLETIN DE LA SOCIÉTÉ MATHÉMATIQUE DE FRANCE 
Ainsi $\mathrm{D}_{G \cdot \sigma} \Gamma_{z}^{G \cdot \sigma}$ est constant sur $\mathcal{U}_{z^{\prime}}$ pour tout $z^{\prime} \in \mathrm{H}^{1}\left(F, \mathrm{Z}\left(G^{\sigma}\right)\right)$. On sait, d'après la proposition 8.1, que lorsque $\sigma$ est unipotent (resp. semi-simple), l'ensemble des éléments réguliers unipotents (resp. pseudo-unipotents) de $G^{F} \cdot \sigma$ est la réunion des $\mathcal{U}_{z^{\prime}}$; on en conclut grâce au (i) de la proposition 7.2 que $\mathrm{D}_{G \cdot \sigma} \Gamma_{z}^{G \cdot \sigma}$ est nul en dehors $\operatorname{des} \mathcal{U}_{z^{\prime}}$. Ainsi, en utilisant la notation $\gamma_{z^{\prime}}$ pour $\gamma_{\mathcal{U}_{z^{\prime}}}$, on peut écrire

$$
\mathrm{D}_{G \cdot \sigma} \Gamma_{z}^{G \cdot \sigma}=\sum_{z^{\prime} \in \mathrm{H}^{1}\left(F, \mathrm{Z}\left(G^{\sigma}\right)\right)} c_{z, z^{\prime}} \gamma_{z^{\prime}}
$$

pour une matrice de coefficients $\left(c_{z, z^{\prime}}\right)$.

D'après le (ii) de la proposition 7.2 , on a

$$
\left\langle\Gamma_{z^{\prime \prime}}^{G \cdot \sigma},(-1)^{|(\Pi / \tau) / \sigma|} \mathrm{D}_{G \cdot \sigma} \Gamma_{z}^{G \cdot \sigma}\right\rangle_{G^{F} \cdot \sigma}=\left|\mathrm{Z}\left(G^{\sigma}\right)^{F}\right| \delta_{z, z^{\prime \prime}} .
$$

On obtient les relations suivantes sur les coefficents $c_{z, z^{\prime}}$ :

$$
\sum_{z^{\prime} \in \mathrm{H}^{1}\left(F, \mathrm{Z}\left(G^{\sigma}\right)\right)} c_{z, z^{\prime}}\left((-1)^{|(\Pi / \tau) / \sigma|} /\left|\mathrm{Z}\left(G^{\sigma}\right)^{F}\right|\right)\left\langle\Gamma_{z^{\prime \prime}}^{G \cdot \sigma}, \gamma_{z^{\prime}}\right\rangle_{G^{F} \cdot \sigma}=\delta_{z, z^{\prime \prime}} .
$$

Ce qui entraîne que $\left(c_{z, z^{\prime}}\right)$ est inversible et a pour inverse la matrice $\left(d_{z, z^{\prime}}\right)$ définie par

$$
d_{z, z^{\prime}}=\left((-1)^{|(\Pi / \tau) / \sigma|} /\left|\mathrm{Z}\left(G^{\sigma}\right)^{F}\right|\right)\left\langle\Gamma_{z^{\prime}}^{G \cdot \sigma}, \gamma_{z}\right\rangle_{G^{F} \cdot \sigma} .
$$

Nous allons maintenant expliciter $\left\langle\Gamma_{z^{\prime}}^{G \cdot \sigma}, \gamma_{z}\right\rangle_{G^{F} \cdot \sigma}$.

Par définition, $\Gamma_{z^{\prime}}^{G \cdot \sigma}=\operatorname{Ind}_{U^{F} \cdot{ }_{\sigma}}^{G^{F} \cdot{ }^{t}}\left({ }^{t} \widetilde{\chi}_{1}\right)$, où $\left({ }^{t} \widetilde{\chi}_{1}\right)$ est l'extension normalisée à $U^{F} \cdot\left\langle{ }^{t} \sigma\right\rangle$ d'un caractère régulier ${ }^{t} \sigma$-stable de $U^{F}$ qui se factorise à travers $U^{F} /\left(U^{*}\right)^{F}$. Ainsi, comme ci-dessus, on peut montrer que $\Gamma_{z^{\prime}}^{G \cdot \sigma}$ est constant sur les ensembles $\mathcal{U}_{z^{\prime \prime}}$.

Cependant $\left\langle\Gamma_{z^{\prime}}^{G \cdot \sigma}, \gamma_{z}\right\rangle_{G^{F} \cdot \sigma}$ est la valeur moyenne de $\Gamma_{z^{\prime}}^{G \cdot \sigma}$ sur $\mathcal{U}_{z}$. Par définition de $t$, l'élément ${ }^{t}\left(u_{0} \cdot \sigma\right)$ appartient à $\mathcal{U}_{z}$, ce qui entraîne immédiatement que $\left\langle\Gamma_{z^{\prime}}^{G \cdot \sigma}, \gamma_{z}\right\rangle_{G^{F} \cdot \sigma}=\Gamma_{z^{\prime}}^{G \cdot \sigma}\left({ }^{t}\left(u_{0} \cdot \sigma\right)\right)$.

Calculons donc $\Gamma_{z^{\prime}}^{G \cdot \sigma}\left({ }^{t}\left(u_{0} \cdot \sigma\right)\right)$. Supposons tout d'abord que $z^{-1} z^{\prime}$ ne soit pas dans $\mathcal{Z}$; alors, d'après le lemme 7.6,

$$
\Gamma_{z^{\prime}}^{G \cdot \sigma}\left({ }^{t}\left(u_{0} \cdot \sigma\right)\right)=\operatorname{Ind}_{U^{F} \cdot t^{\prime} \sigma}^{G^{F}}\left({ }^{t^{\prime}} \widetilde{\chi}_{1}\right)\left({ }^{t} u_{0} \cdot{ }^{t} \sigma\right)=0 .
$$

Maintenant, supposons $z^{-1} z^{\prime}$ dans $\mathcal{Z}$. Il existe $t_{0} \in T^{\sigma}$ tel que $\mathcal{L}\left(t_{0}\right) \in z^{-1} z^{\prime}$. Alors $t^{\prime}=t_{0} t$ est un représentant de $z^{\prime},{ }^{t^{\prime}} \sigma={ }^{t} \sigma$ et ${ }^{t^{\prime}} \chi_{1}$ est ${ }^{t} \sigma$-stable.

$$
\begin{aligned}
\Gamma_{z^{\prime}}^{G \cdot \sigma}\left({ }^{t}\left(u_{0} \cdot \sigma\right)\right) & =\operatorname{Ind}_{U^{F} \cdot t_{\sigma}}^{G^{F} \cdot{ }^{t}}\left({ }^{t^{\prime}} \widetilde{\chi}_{1}\right)\left({ }^{t}\left(u_{0} \cdot \sigma\right)\right) \\
& =1 /\left|U^{F}\right|_{\left\{g \in G^{F} ; g\left({ }^{t} u_{0}\right)\left({ }^{t} \sigma\right)(g)^{-1} \in U^{F}\right\}}\left({ }^{t^{\prime}} \widetilde{\chi}_{1}\right)\left(g\left({ }^{t} u_{0} \cdot{ }^{t} \sigma\right) g^{-1}\right) .
\end{aligned}
$$

Lemme 8.5. - Soit $g \in G^{F}$ tel que $g\left({ }^{t} u_{0} \cdot{ }^{t} \sigma\right) g^{-1} \in U \cdot{ }^{t} \sigma$, alors $g \in\left(T^{\sigma}\right)^{F} U^{F}$. 
Démonstration. - Soit $g \in G^{F}$. On suppose que l'on a $\left(g\left({ }^{t} u_{0} \cdot{ }^{t} \sigma\right) g^{-1} \in U \cdot{ }^{t} \sigma\right)$. Comme $B$ est un sous-groupe de Borel $\sigma$-stable, $B$ est aussi ${ }^{t} \sigma$-stable et on a $\mathrm{N}_{\widetilde{G}}(B)=B \cdot\left\langle{ }^{t} \sigma\right\rangle$. Donc on a $g\left({ }^{t} u_{0} \cdot{ }^{t} \sigma\right) g^{-1} \in \mathrm{N}_{\widetilde{G}}(B)$, ce qui entraîne que ${ }^{t} u_{0} \cdot{ }^{t} \sigma$ normalise le sous-groupe de Borel $g^{-1} B$. Cependant, comme ${ }^{t} u_{0} \cdot{ }^{t} \sigma$ est régulier, il ne normalise qu'un seul sous-groupe de Borel. Ainsi on a ${ }^{g^{-1}} B=B$ et $g \in B^{F}$.

On pose $g=\tau v$ avec $\tau \in T^{F}$ et $v \in U^{F}$. Un calcul rapide montre que si on a $\tau v\left({ }^{t} u_{0} \cdot{ }^{t} \sigma\right)(\tau v)^{-1} \in U \cdot{ }^{t} \sigma$, alors $\tau \in\left(T^{\sigma}\right)^{F}$. D'où le lemme 8.5

D'après le lemme précédent,

$$
\begin{aligned}
& \Gamma_{z^{\prime}}^{G \cdot \sigma}\left({ }^{t}\left(u_{0} \cdot \sigma\right)\right)=1 /\left|U^{F}\right| \sum_{\tau \in\left(T^{\sigma}\right)^{F}, u \in U^{F}}\left({ }^{t^{\prime}} \tilde{\chi}_{1}\right)\left(u\left({ }^{\tau t} u_{0} \cdot{ }^{t} \sigma\right) u^{-1}\right) \\
& =\sum_{\tau \in\left(T^{\sigma}\right)^{F}}\left({ }^{t^{\prime}} \tilde{\chi}_{1}\right)\left({ }^{\tau t} u_{0} \cdot{ }^{t} \sigma\right) \\
& =\sum_{\tau \in\left(T^{\sigma}\right)^{F}}{ }^{t^{\prime}} \chi_{1}\left({ }^{\tau t} u_{0}\right) \quad \operatorname{car}^{t} \sigma={ }^{t^{\prime}} \sigma \\
& =\sum_{\tau \in\left(T^{\sigma}\right)^{F}}{ }^{\tau t_{0}} \chi_{1}\left(u_{0}\right)=\left|\mathrm{Z}\left(G^{\sigma}\right)^{F}\right| \sigma_{z^{-1} z^{\prime}} .
\end{aligned}
$$

La dernière égalité provient du lemme suivant :

LEMME 8.6. - Soit $z_{0} \in \mathcal{Z}$ et soit $t_{0} \in T^{\sigma}$ tel que $t_{0}^{-1} F\left(t_{0}\right) \in z_{0}$, alors on a $\sigma_{z}=\left|\mathrm{Z}\left(G^{\sigma}\right)^{F}\right|^{-1} \sum_{\tau \in\left(T^{\sigma}\right)^{F}}{ }^{\tau t_{0}} \chi_{1}\left(u_{0}\right)$.

Démonstration. - Par définition, on a $\sigma_{z_{0}}=\sum_{\chi \in \Phi_{z_{0}}} \tilde{\chi}\left(u_{0} \cdot \sigma\right)$, ce que l'on peut écrire $\sigma_{z_{0}}=\sum_{\chi \in \Phi_{z_{0}}} \chi\left(u_{0}\right)$ car tous les caractères de $\Phi_{z_{0}}$ sont $\sigma$-stables. Comme $\Phi_{z_{0}}$ est la $\left(T^{\sigma}\right)^{F}$-orbite de ${ }^{t_{0}} \chi_{1}$, il suffit de montrer que pour $\tau \in\left(T^{\sigma}\right)^{F}$, on a ${ }^{\tau t_{0}} \chi_{1}\left(u_{0}\right)={ }^{t_{0}} \chi_{1}\left(u_{0}\right)$ si et seulement si $\tau \in \mathrm{Z}\left(G^{\sigma}\right)^{F}$. Mais ${ }^{\tau t_{0}} \chi_{1}\left(u_{0}\right)=$ ${ }^{t_{0}} \chi_{1}\left(u_{0}\right)$ signifie que ${ }^{\tau^{-1}} u_{0}=u_{0}$, c'est-à-dire, comme $\tau$ est $\sigma$-stable que $\tau$ appartient au centralisateur dans $G$ de $u_{0} \cdot \sigma$. Mais comme $u_{0} \cdot \sigma$ est régulier, on a $\mathrm{C}_{G}\left(u_{0} \cdot \sigma\right) \cap\left(T^{\sigma}\right)^{F}=\mathrm{Z}\left(G^{\sigma}\right)^{F}$, d'où le résultat.

L'inverse $\left(d_{z, z^{\prime}}\right)$ de la matrice $\left(c_{z, z^{\prime}}\right)$ est donc défini par

$$
d_{z, z^{\prime}}= \begin{cases}(-1)^{|(\Pi / \tau) / \sigma|} \sigma_{z^{-1} z^{\prime}} & \text { si } z^{-1} z^{\prime} \in \mathcal{Z} \\ 0 & \text { sinon. }\end{cases}
$$

La relation $\mathrm{D}_{G \cdot \sigma} \Gamma_{z}^{G \cdot \sigma}=\sum_{z^{\prime} \in \mathrm{H}^{1}\left(F, \mathrm{Z}\left(G^{\sigma}\right)\right)} c_{z, z^{\prime}} \gamma_{z^{\prime}}$ peut être inversée et on obtient

$$
\gamma_{z}=(-1)^{|(\Pi / \tau) / \sigma|} \sum_{\left\{z^{\prime} \in \mathrm{H}^{1}\left(F, \mathrm{Z}\left(G^{\sigma}\right)\right) ; z^{-1} z^{\prime} \in \mathcal{Z}\right\}} \sigma_{z^{-1} z^{\prime}} \mathrm{D}_{G \cdot \sigma} \Gamma_{z^{\prime}}^{G \cdot \sigma},
$$

ce qui entraîne que

$$
\left\langle\gamma_{z}, \tilde{\chi}\right\rangle_{G^{F} \cdot \sigma}=(-1)^{|(\Pi / \tau) / \sigma|} \sum_{\left\{z^{\prime} \in \mathrm{H}^{1}\left(F, \mathrm{Z}\left(G^{\sigma}\right)\right) ; z^{-1} z^{\prime} \in \mathcal{Z}\right\}} \sigma_{z^{-1} z^{\prime}}\left\langle\mathrm{D}_{G \cdot \sigma} \Gamma_{z^{\prime}}^{G \cdot \sigma}, \tilde{\chi}\right\rangle_{G^{F} \cdot \sigma}
$$

BULlEtin DE LA SOCIÉtÉ MATHÉMATIQUE DE FRANCE 
On obtient alors le (i) du théorème 8.4 :

$$
\begin{aligned}
& \frac{1}{\left|\mathcal{U}_{z}\right|} \sum_{u \cdot \sigma \in \mathcal{U}_{z}} \tilde{\chi}(u \cdot \sigma) \\
& =(-1)^{|(\Pi / \tau) / \sigma|} \sum_{\left\{z^{\prime} \in \mathrm{H}^{1}\left(F, \mathrm{Z}\left(G^{\sigma}\right)\right) \mid z^{-1} z^{\prime} \in \mathcal{Z}\right\}} \sigma_{z^{-1}} z^{\prime}\left\langle\Gamma_{z^{\prime}}^{G \cdot \sigma}, \mathrm{D}_{G \cdot \sigma} \tilde{\chi}\right\rangle_{G^{F} \cdot \sigma}
\end{aligned}
$$

Nous allons maintenant démontrer le (ii) du théorème 8.4 :

$$
\begin{aligned}
&\left\langle\sum_{z} \Gamma_{z}^{G \cdot \sigma}, \sum_{z} \Gamma_{z}^{G \cdot \sigma}\right\rangle_{G^{F} \cdot \sigma}=\left\langle\sum_{z} \mathrm{D}_{G \cdot \sigma} \Gamma_{z}^{G \cdot \sigma}, \sum_{z} \mathrm{D}_{G \cdot \sigma} \Gamma_{z}^{G \cdot \sigma}\right\rangle_{G^{F} \cdot \sigma} \\
&=\left\langle\sum_{z, z^{\prime}} c_{z, z^{\prime}} \gamma_{z^{\prime}}, \sum_{z, z^{\prime}} c_{z, z^{\prime}} \gamma_{z^{\prime}}\right\rangle_{G^{F} \cdot \sigma} \\
&(\text { en reprenant les notations du (i) }) \\
&=\sum_{z^{\prime}}\left\langle\sum_{z} c_{z, z^{\prime}} \gamma_{z^{\prime}}, \sum_{z} c_{z, z^{\prime}} \gamma_{z^{\prime}}\right\rangle_{G^{F} \cdot \sigma} \\
&\left(\operatorname{car}\left\langle\gamma_{z}, \gamma_{z^{\prime}}\right\rangle_{G^{F} \cdot \sigma}=0 \text { si } z \neq z^{\prime}, \text { les } \mathcal{U}_{z} \text { étant disjoints }\right) .
\end{aligned}
$$

Nous allons montrer que $\sum_{z} c_{z, z^{\prime}}=1$ en utilisant les lemmes 8.7 à 8.10 ; cela revient à montrer que $\sum_{z^{\prime}} c_{1, z^{\prime}}=1$ car on a $c_{z, z^{\prime}}=c_{1, z^{-1} z^{\prime}}$ (la matrice $\left(d_{z, z^{\prime}}\right)$ vérifie en effet $d_{z, z^{\prime}}=d_{1, z^{-1} z^{\prime}}$ pour tout $z, z^{\prime} \in \mathrm{H}^{1}\left(F, \mathrm{Z}\left(G^{\sigma}\right)\right)$; c'est donc encore vrai pour la matrice inverse $\left(c_{z, z^{\prime}}\right)$ ).

Dans [4], le caractère de Steinberg de $\widetilde{G}^{F}$ est défini comme le dual de l'identité, comme dans le cas connexe. On note alors $\mathrm{St}_{G \cdot \sigma}=\mathrm{D}_{G \cdot \sigma} \operatorname{Id}_{G \cdot \sigma}$, la restriction du caractère de Steinberg à $G^{F} \cdot \sigma$. On a

$$
\begin{aligned}
\sum_{z^{\prime}} c_{1, z^{\prime}} & =\left\langle\mathrm{D}_{G \cdot \sigma} \Gamma_{1}^{G \cdot \sigma}, \operatorname{Id}_{G \cdot \sigma}\right\rangle=\left\langle\Gamma_{1}^{G \cdot \sigma}, \mathrm{St}_{G \cdot \sigma}\right\rangle \\
& =\frac{1}{\left|G^{F}\right|} \sum_{g \in G^{F}} \Gamma_{1}^{G \cdot \sigma}(g \cdot \sigma) \overline{\operatorname{St}_{G \cdot \sigma}(g \cdot \sigma)}
\end{aligned}
$$

Or $\Gamma_{1}^{G \cdot \sigma}(g \cdot \sigma)=\operatorname{Ind}_{U^{F} \cdot \sigma}^{G^{F} \cdot \sigma} \tilde{\chi}_{1}$ est nul si $g \cdot \sigma$ n'est pas conjugué sous $G^{F}$ à un élément de $U^{F} \cdot \sigma$. Par ailleurs, $\operatorname{St}_{G} \cdot \sigma(g \cdot \sigma)=0$ si $g \cdot \sigma$ n'est pas quasi-semisimple (voir [4, prop. 3.18]).

LEMME 8.7. - Lorsque $\sigma$ est unipotent (resp. semi-simple), si $g \cdot \sigma$ est à la fois unipotent (resp. pseudo-unipotent) et quasi-semi-simple, alors $g \cdot \sigma$ est conjugué sous $G \grave{a} \sigma$.

Démonstration. — Le cas unipotent est traité par [7, prop. II.2.21]. Considérons le cas où $\sigma$ est semi-simple. D'après le corollaire 3.12, comme $g \cdot \sigma$ est pseudo-unipotent, il existe $x \in G$ et $u \in U^{\sigma}$ tels que $g \cdot \sigma={ }^{x}(u \cdot \sigma)$. Alors $u \cdot \sigma$ est quasi-semi-simple et il existe un couple $T^{\prime} \subset B^{\prime}$ formé d'un tore maximal

TOME $132-2004-\mathrm{N}^{\mathrm{O}} 2$ 
inclus dans un sous-groupe de Borel de $G$ stables par conjugaison par $u \cdot \sigma$. Comme $u$ est unipotent, $\sigma$ est semi-simple et comme $u$ et $\sigma$ commutent, $u$ est la partie unipotente et $\sigma$ la partie semi-simple de la décomposition de Jordan de $u \cdot \sigma$. Ainsi, le fait que $u \cdot \sigma \in \mathrm{N}_{G}\left(B^{\prime}\right)$ (resp. $\mathrm{N}_{G}\left(T^{\prime}, B^{\prime}\right)$ ) entraîne que $u$ et $\sigma$ sont dans $\mathrm{N}_{G}\left(B^{\prime}\right)$ (resp. $\left.\mathrm{N}_{G}\left(T^{\prime}, B^{\prime}\right)\right)$. Donc, $B^{\prime}$ et $T^{\prime}$ sont $\sigma$-stables et $u \in T^{\prime}$. Ainsi $u$ est à la fois unipotent et semi-simple, ce qui entraîne que $u=1$.

D'après la proposition 3.21 de [3], si $h \in G$, alors $g \cdot \sigma={ }^{h} \sigma$ est rationnel si et seulement si $h^{-1} F(h) \in G^{\sigma}$ et les $G^{F}$-classes de conjugaison de $\mathrm{Cl}_{G}(\sigma)^{F}$ sont paramétrées par $\mathrm{H}^{1}\left(F, G^{\sigma} /\left(G^{\sigma}\right)^{0}\right)=\mathrm{H}^{1}\left(F, \mathrm{Z}\left(G^{\sigma}\right) / \mathrm{Z}\left(\left(G^{\sigma}\right)^{0}\right)\right.$ ) (théorème 2.3, (ii)). Pour toute $F$-classe $z^{\prime} \in \mathrm{H}^{1}\left(F, \mathrm{Z}\left(G^{\sigma}\right) / \mathrm{Z}\left(\left(G^{\sigma}\right)^{0}\right)\right)$, on choisit un élément $t^{\prime}$ dans $\mathcal{L}_{T}^{-1}\left(\mathrm{Z}\left(G^{\sigma}\right)\right)$ tel que l'image de $\mathcal{L}_{T}\left(t^{\prime}\right)=t^{\prime-1} F\left(t^{\prime}\right)$ dans $\mathrm{Z}\left(G^{\sigma}\right) / \mathrm{Z}\left(\left(G^{\sigma}\right)^{0}\right)$ soit dans $z^{\prime}$. On note $\mathcal{E}$ l'ensemble de ces représentants et on obtient

$$
\sum_{z^{\prime}} c_{1, z^{\prime}}=\frac{1}{\left|G^{F}\right|} \sum_{t^{\prime} \in \mathcal{E}}\left|\mathrm{Cl}_{G^{F}}\left({ }^{t^{\prime}} \sigma\right)\right| \Gamma_{1}^{G \cdot \sigma}\left({ }^{t^{\prime}} \sigma\right) \overline{\operatorname{St}_{G \cdot \sigma}\left({ }^{\prime} \sigma\right)},
$$

c'est-à-dire, d'après [4, prop. 3.18],

$$
\sum_{z^{\prime}} c_{1, z^{\prime}}=\frac{1}{\left|G^{F}\right|} \sum_{t^{\prime} \in \mathcal{E}}\left|\mathrm{Cl}_{G^{F}}\left({ }^{t^{\prime}} \sigma\right)\right| \Gamma_{1}^{G \cdot \sigma}\left({ }^{\prime} \sigma\right)\left|\left(\left(G^{t^{\prime}} \sigma\right)^{0}\right)^{F}\right|_{p} .
$$

(Lorsque $\sigma$ est unipotent, $G^{\sigma}$ est connexe et $\mathcal{E}=\{1\}$.)

LEMmE 8.8. - Soit $t^{\prime} \in \mathcal{L}_{T}^{-1}\left(\mathrm{Z}\left(G^{\sigma}\right)\right)$ tel que l'image de $\mathcal{L}_{T}\left(t^{\prime}\right)=t^{\prime-1} F\left(t^{\prime}\right)$ dans $\mathrm{Z}\left(G^{\sigma}\right) / \mathrm{Z}\left(\left(G^{\sigma}\right)^{0}\right)$ ne soit pas dans la $F$-classe triviale de $\mathrm{Z}\left(G^{\sigma}\right) / \mathrm{Z}\left(\left(G^{\sigma}\right)^{0}\right)$. Alors on a $\Gamma_{1}^{G \cdot \sigma}\left(t^{\prime} \sigma\right)=0$.

Démonstration. - On a

$$
\Gamma_{1}^{G \cdot \sigma}\left(t^{\prime} \sigma\right)=\operatorname{Ind}_{U^{F} \cdot \sigma}^{G^{F} \cdot \sigma} \widetilde{\chi}_{1}\left({ }^{t^{\prime}} \sigma\right)=\frac{1}{\left|U^{F}\right|} \sum_{\left\{x \in G^{F} ; x^{\left.t^{\prime} \sigma x^{-1} \in U^{F} \cdot \sigma\right\}}\right.} \tilde{\chi}_{1}\left(x^{\left.t^{\prime} \sigma x^{-1}\right) .}\right.
$$

Il suffit donc de montrer que $\mathrm{Cl}_{G^{F}}\left({ }^{\prime} \sigma\right) \cap U \cdot \sigma$ est vide. Or :

LEMME 8.9. - Si $\sigma$ est un élément quasi-central rationnel du quasi-tore $\mathrm{N}_{G}(T, B)$, alors $\mathrm{Cl}_{G}(\sigma)^{F} \cap U \cdot \sigma=\mathrm{Cl}_{U^{F}}(\sigma)$.

Démonstration. - On va d'abord montrer que $\mathrm{Cl}_{G}(\sigma) \cap B \cdot \sigma=\mathrm{Cl}_{B}(\sigma)$.

Soit $x \in G$ tel que $x \cdot \sigma x^{-1} \in B \cdot \sigma$. Alors le sous-groupe de Borel $x^{-1} B$ est $\sigma$-stable. Le sous-groupe de ${ }^{x^{-1}} B$ contient un tore maximal $\sigma$-stable $T^{\prime}$ ( $c f$. [4, prop. $1.11(\mathrm{i})])$ et les couples $T \subset B$ et $T^{\prime} \subset{ }^{x^{-1}} B$ sont conjugués sous $G^{\sigma}$ (cf. [4, prop. 1.21]). Donc il existe $g \in G^{\sigma}$ tel que ${ }^{x^{-1} B}={ }^{g} B$, c'est-à-dire tel que $x g \in B$ et si on pose $b=x g$, alors $x \sigma x^{-1}=b \sigma b^{-1}$. D'où l'égalité $\mathrm{Cl}_{G}(\sigma) \cap B \cdot \sigma=\mathrm{Cl}_{B}(\sigma)$.

BULletin DE LA SOCIÉtÉ MATHÉMATIQUE DE FRANCE 
Montrons maintenant que $\mathrm{Cl}_{B}(\sigma) \cap U \cdot \sigma=\mathrm{Cl}_{U}(\sigma)$. Soit $b \in B$ tel que $b \sigma b^{-1}=u \cdot \sigma \in U \cdot \sigma$. On peut écrire de façon unique $b=t v$ avec $t \in t$ et $v \in U$. Un calcul rapide montre que si $b \sigma b^{-1}=u \cdot \sigma$, alors on a $b \sigma b^{-1} \in \mathrm{Cl}_{U}(\sigma)$.

On a donc $\mathrm{Cl}_{G}(\sigma)^{F} \cap U \cdot \sigma=\mathrm{Cl}_{U}(\sigma)^{F}$. Or, d'après [3, prop. 3.21], les $U^{F}$ classes de conjugaisons de $\mathrm{Cl}_{U}(\sigma)^{F}$ sont paramétrées par $\mathrm{H}^{1}\left(F, U^{\sigma}\right)$ et comme $U^{\sigma}$ est connexe, on a $\mathrm{Cl}_{U}(\sigma)^{F}=\mathrm{Cl}_{U^{F}}(\sigma)$. D'où $\mathrm{Cl}_{G}(\sigma)^{F} \cap U \cdot \sigma=\mathrm{Cl}_{U^{F}}(\sigma)$.

D'après le lemme 8.9 ci-dessus, on a $\mathrm{Cl}_{G}(\sigma)^{F} \cap U \cdot \sigma=\mathrm{Cl}_{G^{F}}(\sigma) \cap U \cdot \sigma$. Donc $\mathrm{Cl}_{G^{F}}\left({ }^{\prime} \sigma\right) \cap U \cdot \sigma \subset \mathrm{Cl}_{G^{F}}(\sigma)$; or on a vu plus haut que les $G^{F_{-}}$ classes de conjugaison de $\mathrm{Cl}_{G}(\sigma)^{F}$ sont paramétrées par $\mathrm{H}^{1}\left(F, G^{\sigma} /\left(G^{\sigma}\right)^{0}\right)=$ $\mathrm{H}^{1}\left(F, \mathrm{Z}\left(G^{\sigma}\right) / \mathrm{Z}\left(\left(G^{\sigma}\right)^{0}\right)\right)$. En particulier, la classe $\mathrm{Cl}_{G^{F}}\left({ }^{t^{\prime}} \sigma\right)$ est la $G^{F}$-classe paramétrée par la $F$-classe $z^{\prime}$ de l'image de $t^{\prime-1} F\left(t^{\prime}\right)$ dans $\mathrm{Z}\left(G^{\sigma}\right) / \mathrm{Z}\left(\left(G^{\sigma}\right)^{0}\right)$; elle est distincte de $\mathrm{Cl}_{G^{F}}(\sigma)$, car $z^{\prime}$ n'est pas triviale. Donc $\mathrm{Cl}_{G^{F}}\left({ }^{\prime} \sigma\right) \cap U \cdot \sigma$ est vide. On en déduit le lemme 8.8.

Revenons à la démonstration du (ii) du théorème 8.4. Le lemme 8.8 permet d'écrire que

$$
\sum_{z^{\prime}} c_{1, z^{\prime}}=\frac{1}{\left|G^{F}\right|}\left|\mathrm{Cl}_{G^{F}}(\sigma)\right| \cdot \Gamma_{1}^{G \cdot \sigma}(\sigma) \cdot\left|\left(\left(G^{\sigma}\right)^{0}\right)^{F}\right|_{p}
$$

(Le résultat est évident lorsque $\sigma$ est unipotent et le lemme 8.8 est alors inutile.) Nous allons calculer $\Gamma_{1}^{G \cdot \sigma}(\sigma)$ :

$$
\Gamma_{1}^{G \cdot \sigma}(\sigma)=\operatorname{Ind}_{U^{F} \cdot \sigma}^{G^{F} \cdot \sigma} \tilde{\chi}_{1}(\sigma)=\frac{1}{\left|U^{F}\right|} \sum_{\left\{x \in G^{F} ; x \sigma x^{-1} \in U^{F} \cdot \sigma\right\}} \tilde{\chi}_{1}\left(x \sigma x^{-1}\right) .
$$

LEMME 8.10. - Soit $x \in G^{F}$; alors on a $x \sigma(x)^{-1} \in U^{F}$ si et seulement si $x \in U^{F}\left(G^{\sigma}\right)^{F}$.

Démonstration. - D'après le lemme 8.9, on a $\mathrm{Cl}_{G^{F}}(\sigma) \cap U \cdot \sigma=\mathrm{Cl}_{U^{F}}(\sigma)$. Ainsi, si $x \in G^{F}$ est tel que $x \sigma(x)^{-1} \in U^{F}$, alors il existe $v \in U^{F}$ tel que $x \sigma x^{-1}=v \sigma v^{-1}$, c'est-à-dire tel que $x v^{-1} \in G^{\sigma}$. La réciproque est évidente.

Ainsi, $\Gamma_{1}^{G \cdot \sigma}(\sigma)=\left|U^{F}\right|^{-1} \cdot\left|U^{F}\right| \cdot\left|\left(G^{\sigma}\right)^{F}\right| \cdot\left|\left(U^{\sigma}\right)^{F}\right|^{-1}=\left|\left(G^{\sigma}\right)^{F}\right| \cdot\left|\left(U^{\sigma}\right)^{F}\right|^{-1}$. Et l'on obtient comme annoncé

$$
\sum_{z^{\prime}} c_{1, z^{\prime}}=\frac{1}{\left|G^{F}\right|} \cdot\left|\mathrm{Cl}_{G^{F}}(\sigma)\right| \cdot\left|\left(G^{\sigma}\right)^{F}\right| \cdot\left|\left(U^{\sigma}\right)^{F}\right|^{-1} \cdot\left|\left(\left(G^{\sigma}\right)^{0}\right)^{F}\right|_{p}=1 .
$$

La dernière égalité vient du fait que $\left|G^{F}\right|=\left|\mathrm{Cl}_{G^{F}}(\sigma)\right| \cdot\left|\left(G^{F}\right)^{\sigma}\right|$ et du fait que $\left|\left(\left(G^{\sigma}\right)^{0}\right)^{F}\right|_{p}=\left|\left(U^{\sigma}\right)^{F}\right|$, ce dernier résultat étant de [3, prop. 3.19].

Terminons maintenant la démonstration de 8.4 (ii). On a :

$$
\left\langle\sum_{z} \Gamma_{z}^{G \cdot \sigma}, \sum_{z} \Gamma_{z}^{G \cdot \sigma}\right\rangle_{G^{F} \cdot \sigma}=\sum_{z^{\prime}}\left\langle\gamma_{z^{\prime}}, \gamma_{z^{\prime}}\right\rangle_{G^{F} \cdot \sigma}=\sum_{z^{\prime}}\left|G^{F}\right| /\left|\mathcal{U}_{z^{\prime}}\right|
$$

TOME $132-2004-\mathrm{N}^{\mathrm{O}} 2$ 
Or, si $z$ et $z^{\prime}$ sont deux éléments de $\mathrm{H}^{1}\left(F, \mathrm{Z}\left(G^{\sigma}\right)\right)$ et si $t$ et $t^{\prime}$ sont respectivement des représentants dans $\mathcal{L}_{T^{\sigma}}^{-1}\left(\mathrm{Z}\left(G^{\sigma}\right)\right)$ de $z$ et $z^{\prime}$, alors $\mathcal{U}_{z^{\prime}}={ }^{t^{\prime}} t^{-1} \mathcal{U}_{z}$. Donc les $\mathcal{U}_{z}$ ont tous le même cardinal.

Calculons tout d'abord le cardinal de $\mathcal{U}_{z}$ lorsque $\sigma$ est unipotent. Selon le (ii) de la proposition 8.1, l'ensemble des éléments réguliers unipotents de $G^{F} \cdot \sigma$ est la réunion des $\mathcal{U}_{z}$, donc le cardinal de n'importe quel $\mathcal{U}_{z}$ est égal au nombre d'éléments réguliers unipotents de $G^{F} \cdot \sigma$ divisé par le cardinal de $\mathrm{H}^{1}\left(F, \mathrm{Z}\left(G^{\sigma}\right)\right)$. Ainsi, par le théorème 3.6, on a

$$
\left|\mathcal{U}_{z}\right|=\left|G^{F}\right| /\left(\left|\left(\mathrm{Z}\left(G^{\sigma}\right)^{0}\right)^{F}\right| \times q^{\ell} \times\left|\mathrm{H}^{1}\left(F, \mathrm{Z}\left(G^{\sigma}\right)\right)\right|\right)
$$

où $\ell$ est le rang-semi-simple de $G^{\sigma}$. On peut alors simplifier l'expression de $\left|\mathcal{U}_{z}\right|$ en utilisant le fait que $\left|\mathrm{H}^{1}\left(F, \mathrm{Z}\left(G^{\sigma}\right)\right)\right|=\left|\mathrm{Z}\left(G^{\sigma}\right)^{F}\right| /\left|\left(\mathrm{Z}\left(G^{\sigma}\right)^{0}\right)^{F}\right|$; on obtient alors

$$
\left|\mathcal{U}_{z}\right|=\left|G^{F}\right| /\left(\left|\mathrm{Z}\left(G^{\sigma}\right)^{F}\right| \times q^{\ell}\right) .
$$

Considérons finalement le cas semi-simple. Il existe $t \in\left(T^{\sigma}\right)^{0}$ tel que

$$
\mathcal{U}_{z}=\left\{x \cdot \sigma \in G^{F} \cdot \sigma ; x \cdot \sigma \sim_{G^{F}}{ }^{t}\left(u_{0}\left(U^{\sigma}\right)^{*}\right) \cdot \sigma\right\} .
$$

Par ailleurs, $\mathcal{U}_{z}$ est une réunion de $G^{F}$-classes unipotentes qui sont paramétrées par $\mathrm{H}^{1}\left(F, \mathrm{C}_{U^{\sigma}}\left(u_{0}\right)\right)$. Pour toute $F$-classe de $\mathrm{C}_{U^{\sigma}}\left(u_{0}\right)$, on peut choisir un représentant dans $U^{\sigma}$; soit $\left\{u_{1}, \ldots, u_{r}\right\}$ un ensemble de tels représentants. Alors $\mathcal{U}_{z}$ est la réunion disjointe des classes $\mathrm{Cl}_{G^{F}}\left({ }^{t u_{i}} u_{0} \cdot \sigma\right)$. Donc, on a

$$
\begin{aligned}
\left|\mathcal{U}_{z}\right| & =\sum_{i}\left|\mathrm{Cl}_{G^{F}}\left({ }^{t u_{i}} u_{0} \cdot \sigma\right)\right| \\
& =\sum_{i}\left|G^{F}\right| /\left|\mathrm{C}_{G^{F}}\left({ }^{t u_{i}} u_{0} \cdot \sigma\right)\right|=\sum_{i}\left|G^{F}\right| /\left|\mathrm{C}_{\left(G^{\sigma}\right)^{F}}\left({ }^{t u_{i}} u_{0}\right)\right| \\
& =\sum_{i}\left(\left|G^{F}\right| /\left|\mathrm{C}_{\left(G^{\sigma}\right)^{0 F}}\left({ }^{t u_{i}} u_{0}\right)\right|\right) \times\left(\left|Z\left(\left(G^{\sigma}\right)^{0}\right)^{F}\right| /\left|Z\left(G^{\sigma}\right)^{F}\right|\right) \\
& =\left(\left|G^{F}\right| /\left|\left(G^{\sigma}\right)^{0 F}\right|\right) \times\left(\left|Z\left(\left(G^{\sigma}\right)^{0}\right)^{F}\right| /\left|Z\left(G^{\sigma}\right)^{F}\right|\right) \sum_{i}\left|\mathrm{Cl}_{\left(G^{\sigma}\right)^{0 F}}\left({ }^{t u_{i}} u_{0}\right)\right| \\
& =\left(\left|G^{F}\right| /\left|\left(G^{\sigma}\right)^{0 F}\right|\right) \times\left(\left|Z\left(\left(G^{\sigma}\right)^{0}\right)^{F}\right| /\left|Z\left(G^{\sigma}\right)^{F}\right|\right) \times\left|\mathcal{U}_{z^{\prime}}^{\prime}\right|
\end{aligned}
$$

où $\mathcal{U}_{z^{\prime}}^{\prime}$ est le sous-ensemble de $\left(G^{\sigma}\right)^{0}$ défini par

$$
\mathcal{U}_{z^{\prime}}^{\prime}=\left\{x \cdot \sigma \in\left(G^{\sigma}\right)^{0 F} ; x \sim_{\left(G^{\sigma}\right)^{0 F}}{ }^{t}\left(u_{0}\left(U^{\sigma}\right)^{*}\right)\right\}
$$

et $z^{\prime} \in \mathrm{H}^{1}\left(F, \mathrm{Z}\left(\left(G^{\sigma}\right)^{0}\right)\right)$ est tel que $t^{-1} F(t) \in z^{\prime}$. Alors la réunion disjointe des $\mathcal{U}_{z}^{\prime}$, pour $z \in \mathrm{H}^{1}\left(F, Z\left(\left(G^{\sigma}\right)^{0}\right)\right)$ est l'ensemble des éléments réguliers unipotents de $\left(G^{\sigma}\right)^{0 F}$ (voir [2, prop. 3.2]). Par ailleurs, les $\mathcal{U}_{z}^{\prime}$ sont géométriquement conjugués et sont donc tous de même cardinal qui est égal au nombre des unipotents réguliers de $\left(G^{\sigma}\right)^{0 F}$ divisé par $\left|\mathrm{H}^{1}\left(F, Z\left(\left(G^{\sigma}\right)^{0}\right)\right)\right|$, c'est-à-dire

$$
\left|\left(G^{\sigma}\right)^{0 F}\right| /\left(\left|\mathrm{H}^{1}\left(F, Z\left(\left(G^{\sigma}\right)^{0}\right)\right)\right| \times\left|Z\left(\left(G^{\sigma}\right)^{0}\right)^{0 F}\right| q^{\ell}\right),
$$

où $\ell$ est le rang semi-simple de $\left(G^{\sigma}\right)^{0}$ (voir [3, prop. 14.23]). 
En utilisant le fait que $\left|\mathrm{H}^{1}\left(F, Z\left(\left(G^{\sigma}\right)^{0}\right)\right)\right|=\left|Z\left(\left(G^{\sigma}\right)^{0}\right)^{F}\right| /\left|Z\left(\left(G^{\sigma}\right)^{0}\right)^{0 F}\right|$, on en déduit le cardinal de $\mathcal{U}_{z}$ lorsque $\sigma$ est semi-simple et on retrouve la même formule que dans le cas unipotent :

$$
\left|\mathcal{U}_{z}\right|=\left|G^{F}\right| /\left(\left|Z\left(G^{\sigma}\right)^{F}\right| q^{\ell}\right) .
$$

Remarque 8.11. - Les calculs ci-dessus montrent que le nombre d'éléments pseudo-unipotents réguliers rationnels de $G^{F} \cdot \sigma$ est égal à $\left|\mathrm{H}^{1}\left(F, Z\left(G^{\sigma}\right)\right)\right|$ multiplié par le cardinal de $\mathcal{U}_{z}$, on retrouve la formule du théorème 3.6 :

$$
\left|G^{F}\right| /\left(\left|Z\left(\left(G^{\sigma}\right)^{0}\right)^{F}\right| q^{\ell}\right)
$$

On peut maintenant terminer les calculs du théorème 8.4

$$
\begin{aligned}
\left\langle\sum_{z} \Gamma_{z}^{G \cdot \sigma}, \sum_{z} \Gamma_{z}^{G \cdot \sigma}\right\rangle_{G^{F} \cdot \sigma} & =\sum_{z^{\prime}}\left|G^{F}\right| /\left|\mathcal{U}_{z^{\prime}}\right| \\
& =\left|\mathrm{H}^{1}\left(F, \mathrm{Z}\left(G^{\sigma}\right)\right)\right| \times\left(\left|G^{F}\right| /\left|\mathcal{U}_{z}\right|\right) \\
& \text { où } z \in \mathrm{H}^{1}\left(F, \mathrm{Z}\left(G^{\sigma}\right)\right) \text { est quelconque } \\
& =\left|\mathrm{H}^{1}\left(F, \mathrm{Z}\left(G^{\sigma}\right)\right)\right| \times\left|Z\left(G^{\sigma}\right)^{F}\right| q^{\ell} .
\end{aligned}
$$

Nous allons appliquer les résultats précédents au cas où $\mathrm{Z}\left(G^{\sigma}\right)$ est connexe.

Corollaire 8.12. - Supposons $\mathrm{Z}\left(G^{\sigma}\right)$ connexe. Alors on a une unique représentation de Gelfand-Graev de $G \cdot \sigma$ que l'on note $\Gamma$ et $\mathrm{D}_{G \cdot \sigma} \Gamma$ est égal à $\left|\mathrm{Z}\left(G^{\sigma}\right)^{F}\right| q^{\ell}$ sur les éléments réguliers unipotents lorsque $\sigma$ est unipotent et pseudo-unipotents lorsque $\sigma$ est semi-simple (et à zéro ailleurs).

Démonstration. - C'est clair, car dans ce cas $\mathcal{U}_{1}$ est l'ensemble de tous les unipotents (resp. pseudo-unipotents) réguliers et on a $\mathrm{D}_{G \cdot \sigma} \Gamma=\gamma_{\mathcal{U}_{1}}$ en prenant pour $\gamma_{\mathcal{U}_{1}}$ la définition 8.2.

\section{BIBLIOGRAPHIE}

[1] CARter (R.W.) - Finite groups of Lie type, Wiley-Interscience, 1985.

[2] Digne (F.), Lehrer (G.I.) \& Michel (J.) - The characters of the group of rational points of a reductive group with non-connected centre, J. reine angew. Math., t. 425 (1992), pp. 155-192.

[3] Digne (F.) \& Michel (J.) - Representations of Finite Groups of Lie Type, London Math. Soc. Student Texts, vol. 21, Cambridge University Press, Cambridge, 1991.

[4] , Groupes réductifs non connexes, Ann. Sci. École Normale Sup., t. 27 (1994), pp. 345-406.

[5] _ Points fixes des automorphismes quasi-semi-simples, C. R. Acad. Sci. Paris, Sér. I, t. 334 (2002), pp. 1055-1060.

TOME $132-2004-\mathrm{N}^{\mathrm{O}} 2$ 
[6] Malle (G.) - Generalized Deligne-Lusztig characters, J. Alg., t. 159 (1993), no. 1, pp. 64-97.

[7] Spaltenstein (N.) - Classes unipotentes et sous-groupes de Borel, Lectures Notes in Math., vol. 946, Springer, 1982.

[8] Steinberg (R.) - Endomorphisms of linear algebraic groups, Mem. Amer. Math. Soc., vol. 80, American Mathematical Society, Providence, RI, 1968. 